\title{
AN EXAMINATION OF ORGANIZATIONAL LEARNING AND DATA DRIVEN DECISION MAKING WITHIN TWO UNIVERSITY ENROLLMENT MANAGEMENT PROGRAMS
}

A Dissertation

presented to the Faculty of the Graduate School at the University of Missouri-Columbia

In Partial Fulfillment

of the Requirements for the Degree Doctor of Education

\section{By}

\section{J.D. Gragg}

Dr. Barbara Martin, Dissertation Supervisor

December 2019 
(C) Copyright by J D Gragg, 2019 


\begin{abstract}
All Rights Reserved
The undersigned, appointed by the dean of the Graduate School, have examined the dissertation entitled
AN EXAMINATION OF ORGANIZATIONAL LEARNING AND DATA DRIVEN DECISION MAKING WITHIN TWO UNIVERSITY ENROLLMENT MANAGEMENT PROGRAMS

presented by J D Gragg a candidate for the degree of doctor of education, and hereby certify that, in their opinion, it is worthy of acceptance.
\end{abstract}

Dr. Barbara N. Martin, Dissertation Advisor

Dr. Sandy Hutchinson

Dr. Carolyn McKnight

Dr. Doug Thomas 


\section{DEDICATION}

To my boys, Barrett and Brendan, if a job is worth doing, it is worth doing right. 


\section{ACKNOWLEDGEMENTS}

Thank you to Dr. Barbara Martin for her love and support through this process. Your continual encouragement and belief in me, even when I didn't believe in myself, were essential to getting to this point. I learned a great deal from you, both in the classroom and out, about school and life. A great deal of the joy I received from this program came from working with you.

To my wife and sons, thank you for your love and support. Thank you to Sara for showing me the way and how to do school the right way, even if I didn't always or ever follow your path. Thank you for countless nights and weekends taking the boys out of the house so I could write. Thank you for taking care of things so I could travel to do my research and for being there the entire way. Barrett and Brendan, I hope you see the value of education and hard work that your mother and I try to show, if you work hard you can achieve your dreams. Thank you for being my motivation to improve myself to show you it is possible. I love you all!

To my mom, thank you for showing me the value of education. As a lifelong educator and role model, you showed me at a young age that you have to work hard to achieve your goals, and no one else is going to get you there. Thank you for helping with the boys so I can work and for all you have done and continue to do for me and our family.

Finally, to my friends and colleagues within the program and outside the program, thank you for your support and encouragement. Specific thank to the "Magnificent 7" of the Lee's Summit Cohort, you are all awesome, and I learned so much from going on this adventure with you all. I never would have imagined I would gain a best friend from this 
program, especially not an overthinking "Woo." Angela, I would not have completed this program without you, plain and simple. You encouraged me, you motivated me, you helped me with details and big-pictures items, you made the journey fun. I can't thank you enough for all you did and continue to do, and I will always consider our enriched friendship the biggest bonus feature of this degree. 


\section{TABLE OF CONTENTS}

ACKNOWLEDGEMENTS ........................................................

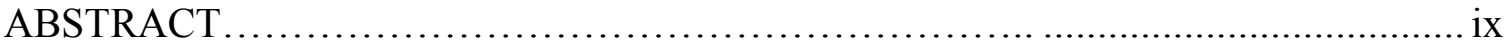

\section{Chapter}

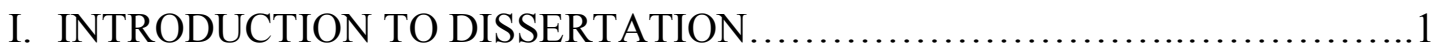

Introduction.........................................................

Statement of the Problem.....................................................

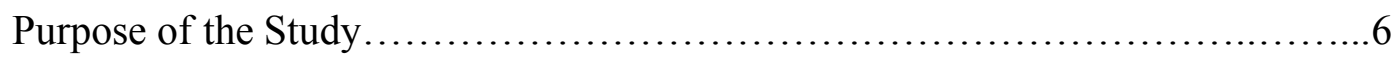

Research Questions...................................................7

Conceptual/Theoretical Frameworks....................................

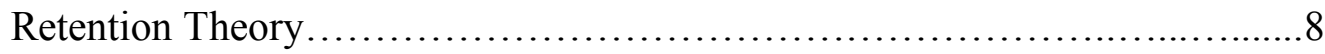

College Choice Model.............................................8

Predictive Modeling...................................................9

Evidence-Based Practice............................................ 10

Data-Driven Decision-Making...................................... 10

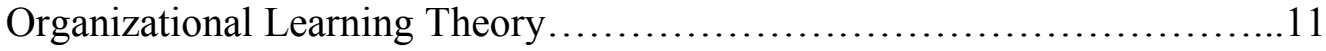

Design for the Study............................................... 12

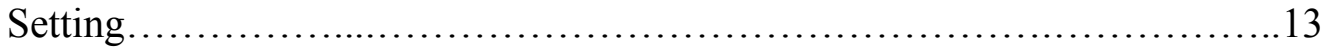

Interview Protocols................................................... 16

Focus Group Protocols............................................17

Documents and Artifacts............................................. 19

Data Analysis Procedures.......................................... 19

Limitations \& Assumptions.................................................21 


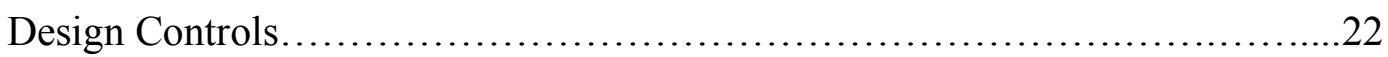

Definition of Key Terms.................................................22

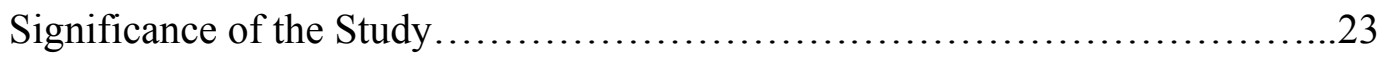

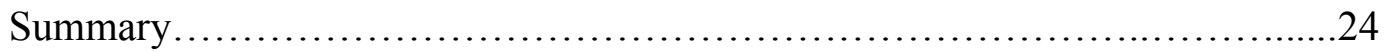

II. PRACTITIONER SETTING FOR THE STUDY ...............................26

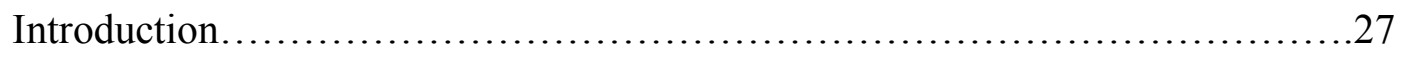

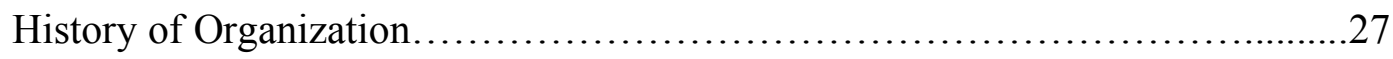

Large Public University ............................................27

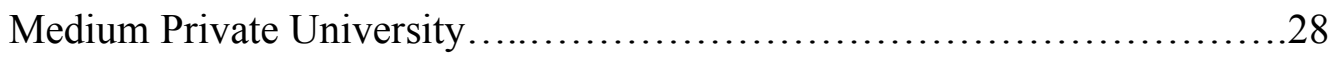

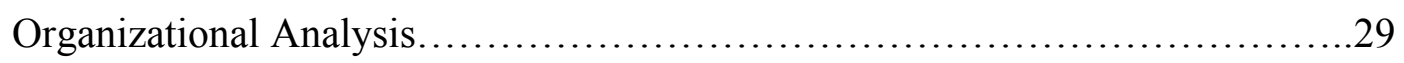

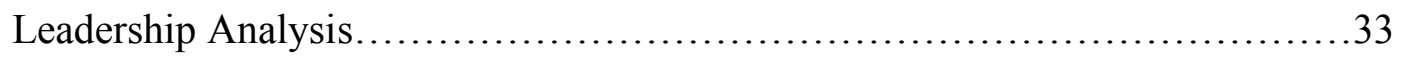

Implications for Research in the Practitioner Setting...........................36

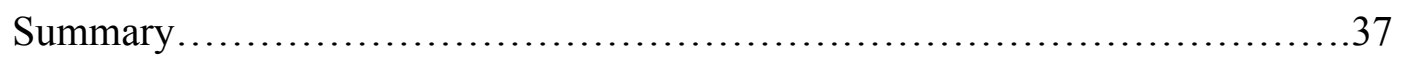

III. SCHOLARLY REVIEW FOR THE STUDY ...................................

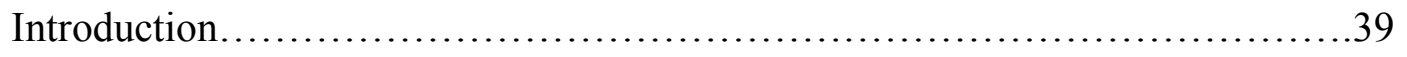

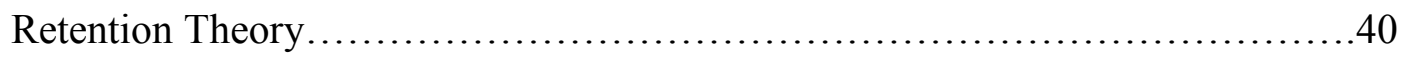

College Choice Model....................................................42

Predictive Modeling.................................................... 45

Evidence-Based Practice...................................................46

Conceptual Frameworks................................................47

Organizational Learning ................................................ 48

Data-Driven Decision Making...........................................56

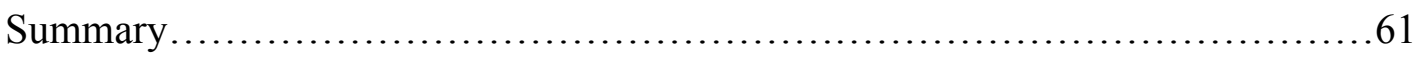




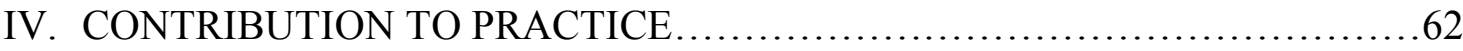

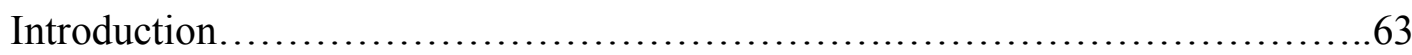

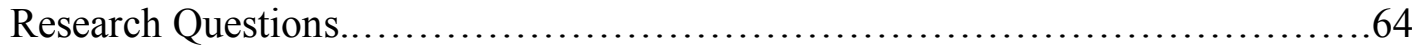

Connection to Conceptual/Theoretical Framework...............................66

Particpants and Data Collection...........................................67

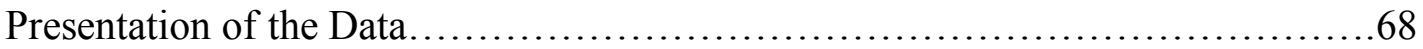

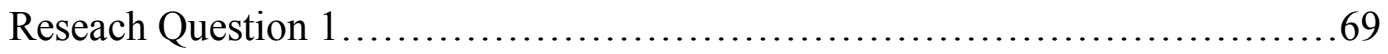

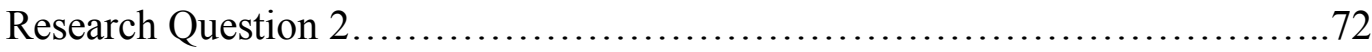

Research Question 3...................................................76

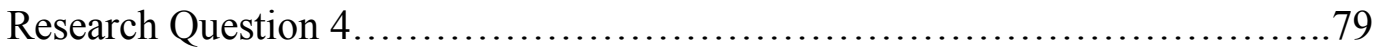

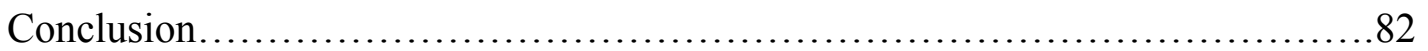

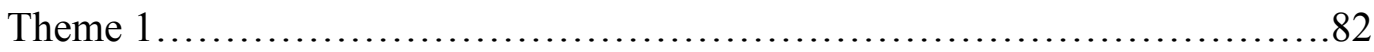

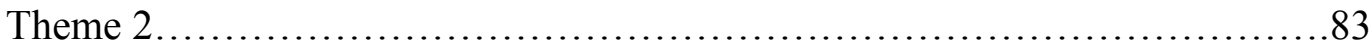

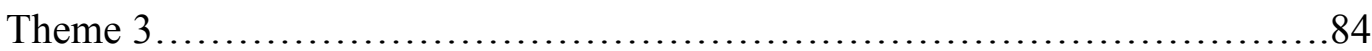

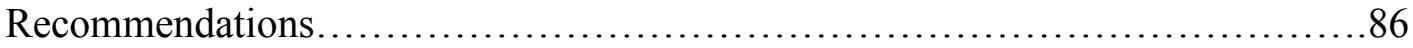

V. CONTRIBUTION TO SCHOLARSHIP .................................. 87

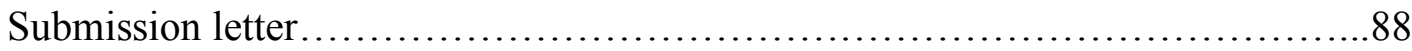

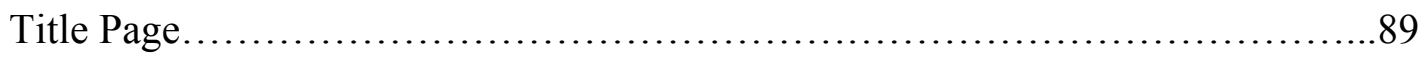

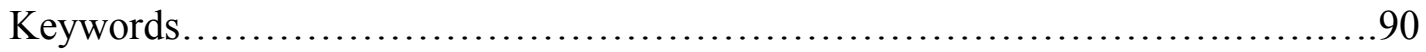

Main Text.............................................................. 90

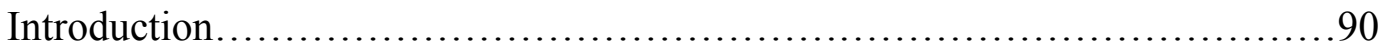

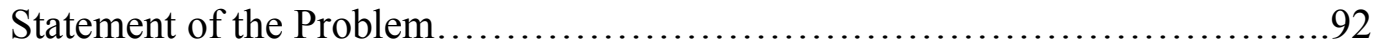




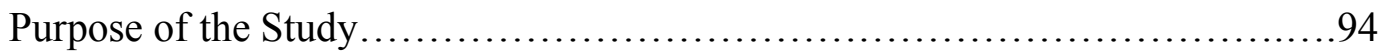

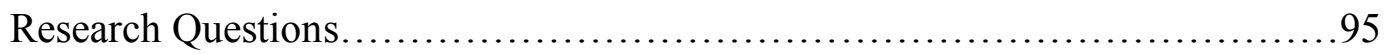

Introduction to the Scholarly Review..................................... 96

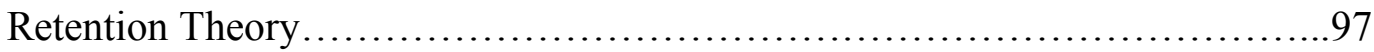

College Choice Model......................................................99

Predictive Modeling ..................................................... 102

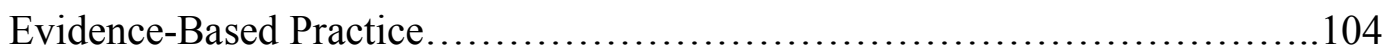

Conceptual Frameworks................................................ 105

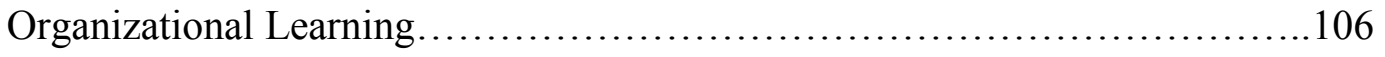

Data-Driven Decision-Making.......................................... 114

Methodological Details................................................... 118

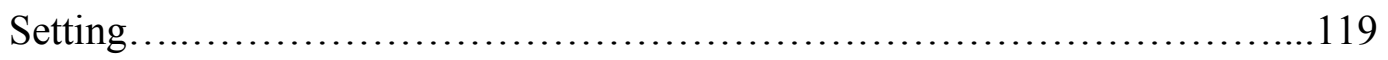

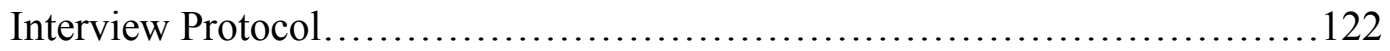

Focus Group Protocol................................................... 123

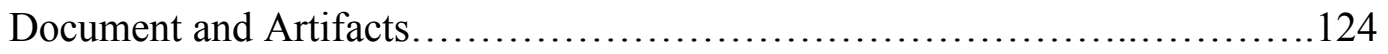

Data Analysis Procedures.............................................. 125

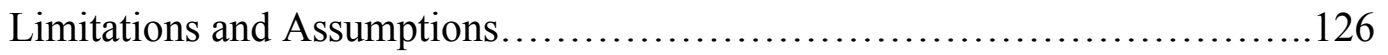

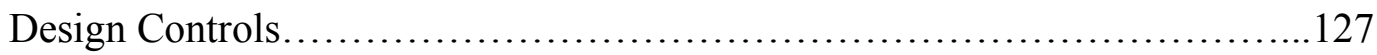

Presentation of the Data................................................. 128

Research Question One.............................................. 130

Research Question Two.................................................133

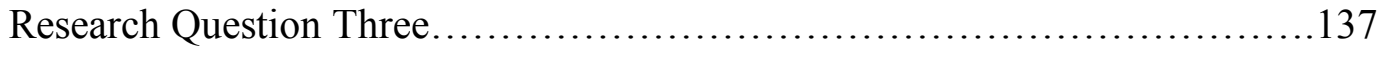

Research Question Four............................................... 140 
Conclusions.

Journal References........................................................... 146

VI. SCHOLARLY PRACTICE REFLECTION ................................ 163

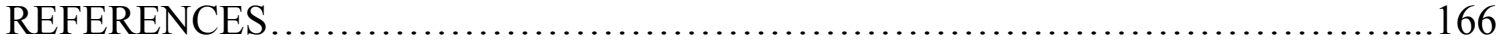

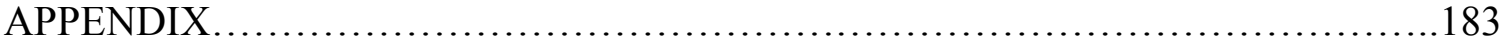

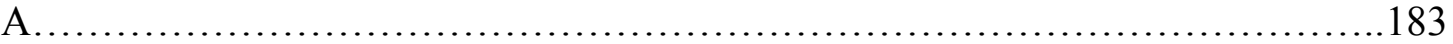

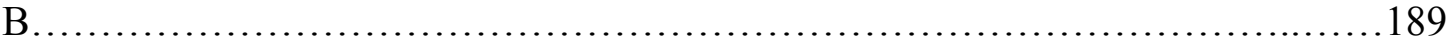

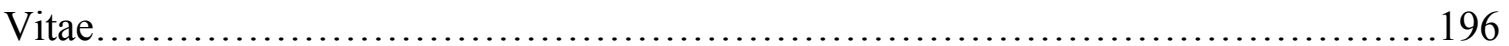




\begin{abstract}
As the number of United States high school graduates continue to decline, research into the most effective ways to recruit and retain college students becomes increasingly valuable. According to the United States Department of Education (Hussar \& Bailey, 2016), students graduating between now and 2028 will decrease by approximately $4.5 \%$. Historically, when focusing on enrollment numbers, college admission offices have recruited students in the door and then let other departments at the university worry about retaining them. In today's competitive market, this is no longer a feasible option.
\end{abstract}

The focus of this research was to address the declining number of students, and the effective strategies universities can use to recruit students who will progress and graduate. Researchers Kretchmar and Memory (2010) and Seeman and O'Hara, (2006) concurred that to retain students is to attempt to understand students beyond the numbers, looking at students as more than a GPA or test score, and understand what the students are desiring in a college and be prepared to sell the student on that experience.

From this research, it appeared that data-driven decision-making can be an effective means to create a successful enrollment management office. Through a practice of data-driven decision-making, organizational learning can happen, which will allow an organization to maintain success and build a culture that sustains that success. Three key themes emerged from the research that will help to inform best practices for other colleges and universities to implement ideas from this research. The three themes are: an organization must have good, usable data and the resources needed to analyze that data; an organization should strive to create a culture of openness, caring, communication, and teamwork to sustain success, and enrollment management is a mix of art and science; an 
organization needs a successful enrollment manager that is a person who can blend the use of data with creating a well-rounded, meaningful, and impactful student recruitment experience. 
SECTION ONE

INTRODUCTION TO DISSERTATION IN PRACTICE 


\section{Introduction}

As the number of United States high school graduates continue to decline, research into the most effective ways to recruit and retain college students becomes increasingly valuable. According to the United States Department of Education (Hussar \& Bailey, 2016), students graduating between now and 2028 will decrease by approximately $4.5 \%$. Thus, all universities will be competing within the same declining pool of students. Although there has been extensive research on best practices in college admission, most of this focuses on qualitative studies with small sample sizes at nonrepresentative or outlier type institutions (Bontrager, 2004). Historically, when focusing on enrollment numbers, college admission offices have recruited students in the door and then let other departments at the university worry about retaining them. In today's competitive market, this is no longer a feasible option. Institutions of higher education should focus on recruiting students who they can retain and graduate (Elliott \& Healy, 2001). Similarly, rising costs associated with college means admissions offices can no longer recruit students who only stay in college a short time. When students do not graduate, the overall net revenue of the university is affected, and schools will struggle to remain fiscally viable (Hussar \& Bailey, 2016).

The focus of this research is to address the declining number of students, and the effective strategies universities can use to recruit students who will progress and graduate. Researchers Kretchmar and Memory (2010) and Seeman and O'Hara, (2006) concurred that to retain students is to attempt to understand students beyond the numbers, looking at students as more than a GPA or test score, and understand what the students are desiring in a college and be prepared to sell the student on that experience. Today's 
students have more choice and power than ever before in their college search, and universities should adapt if they want to remain competitive (Kretchmar \& Memory, 2010; Seeman \& O'Hara, 2006; Stewart \& Heaney, 2013). Therefore, this study will approach the overarching question, How can universities and enrollment management (EM) offices effectively use data to recruit and retain students? Furthermore, how can enrollment management offices learn and adapt to new strategies and make themselves available to learn and create new strategies?

\section{Statement of the Problem}

Within the review of the literature, the identified problem was that there is little to no research on how higher education organizations learn and adapt to use data-driven decision-making. The current research on the use of data-driven decision-making is in the K-12 environment. Legislation has forced K-12 to be more data-driven(Greene \& Forster, 2003; NCLB, 2001). However, the research is lacking when it comes to higher education, as most of the literature discusses the need for using data (Mandinach, 2012; Marsh, Pane, \& Hamilton, 2006), how to collect and disseminate data (Price \& Kirkwood, 2014), and how to use it to inform practice from an institutional level (Sailesh, Lu, \& Al Aali, 2016), not within enrollment management (Hora, Bouwma-Gearhart, \& Park, 2017).

Additionally, there is a literature gap when it comes to using this data within a learning organization.

Often in higher education, there are examples of universities creating a new program due to an initiative from the administration. Consequently, that is not a new process that comes from organizational learning (Cangelosi \& Dill, 1965; R. L. Chapman, Kennedy, Newell, \& Biel, 1959), but rather a system that comes from a 
directive. Researchers Levitt and March (1988) revealed when organizations learn through experience and make changes based on evidence; those changes tend to be lasting and are more profound. While research exists on successful EM strategies and initiatives (Bontrager, 2004; DesJardins, Ahlburg, \& McCall, 2006; Dolence, 1998), there remains a gap in researching ways for those programs to occur organically and without special funding (Jose, Kurian, \& Biju, 2016; Miller \& Bell, 2016).

In an attempt to place this issue into perspective through a review of the literature, the researcher noticed several challenges facing admissions offices, and these challenges have not changed over time. First, as technology and the needs of students change, admissions offices must be willing and able to adapt to the needs of the student (Lindbeck \& Fodrey, 2010). The days of the Field of Dreams model of recruitment, the "we are here, and students will find us model," are over. Even the most selective of schools are having to recruit in ways they never had to in previous years (Hill \& Winston, 2010). Another major challenge is how to replicate best practices at other schools that do not have the same resources. Most research on best practices focuses on programs that have above average resources, regarding personnel and monetary (Bontrager, 2004).

Furthermore, a third challenge is meeting students where they are currently (Ford, 2011; Levine \& Dean, 2013). For example, ten years ago, email was the most effective way to reach students, ten years prior, phone calls and traditional mail, and in present time, texting is what students want. Compounding this problem, students want different types of communication for different types of information. For example, students tend to prefer text communication for data based information such as dates of events. However, they still tend to prefer email or phone calls for informational types of communications 
(Gikas \& Grant, 2013). This study attempted to examine how peer institutions handle these challenges and effectively reach students (Ford, 2011; Lindbeck \& Fodrey, 2010; Mora, 2003; Seeman \& O'Hara, 2006).

Additionally, this research addressed how to connect with special populations of students (Cegler, 2012). Special populations can include first-generation students (Irlbeck, Adams, Akers, Burris, \& Jones, 2014), honor students (Nichols \& Chang, 2013), students in a specific major or field, such as STEM (Moakler \& Kim, 2014) or liberal arts (Baker \& Baldwin, 2015), athletes (Magnusen, Kim, Perrewé, \& Ferris, 2014), dual credit students (Lile, Ottusch, Jones, \& Richards, 2017), transfer students (Foote, Kranzow, \& Hinkle, 2015), and any number of other groups. Unfortunately, these groups present their own recruiting challenges. Different strategies are needed for diverse populations; one size fits all recruitment is no longer relevant (Cegler, 2012; Young \& Johnson, 2004). Finally, additional research is needed that focuses on the best practices that are replicable across various types of institutions and is cost-effective (Francis, 2014).

In this day of reduced funding through all higher education (John, 1990; Tschechtelin, 2011), institutions cannot wait to create change when they receive special funding or new programs. Institutions must create an environment where change can happen organically through organizational learning (Ponnuswamy \& Manohar, 2016), and with evident, data-driven results. Data utilization is the most effective and efficient way to display that something works, at least in the current environment of higher education (Hossler \& Kalsbeek, 2013). Therefore, examining how a higher education EM 
office, specifically an undergraduate admissions office, can learn to use data to create and integrate a predictive model successfully was the focus of this inquiry.

\section{Purpose of the Study}

The purpose of the study using two universities of various size and student population was to examine if and how organizational learning takes place within the EM functions of each school. Use of data-driven decision-making will be considered especially as it relates to success in EM. For purposes of this study, EM success will be defined using metrics the institutions themselves have identified. For example, most public institutions are focused on growing enrollment with academic quality (Baum, Kurose, \& McPherson, 2013). This means these schools want to increase the size of their incoming student population while also increasing the academic profile of their incoming class. Using standardized test scores, high school GPA, and class rank from high school class or other individual measures schools have created can be measured.

Contrary to this, smaller private schools are often less concerned with enrollment growth and more focused on the academic quality of their incoming class (Bontrager, 2004). However, with recent budget cuts to higher education, nearly all institutions are looking to grow enrollment (Calderon \& Mathies, 2013). Therefore, for this research, the primary indicator of EM success was the percentage growth of the incoming freshman and transfer class.

The researcher focused on what types of collected data and what kinds of decisions are made from that data. Specifically, the focus of this was to compare various types of organizational learning that comes from multiple types of data, as well as decision-making models used based on specific data being collected. The examination of 
the use of certain types of data-driven decision making that lead to particular outcomes also occurred with specific regard to the mission of the institution. For example, how do institutions focused on maintaining enrollment compared to growing enrollment make different types of decisions? Do they gather different types of data?

\section{Research Questions}

The following research questions guided this research:

1. How does the use of enrollment data for data-driven decision-making affect the organizational learning of the University, and what type of learning is derived?

2. How can an EM office create a data-driven recruitment model through organizational learning and implement it successfully, and does it perform equally amongst different student populations?

3. What impact does the predictive model have on recruitment and retention, and is that impact equal across different student populations?

4. Do institutions that have successful EM offices rely solely on data-driven decision-making, or are there elements of evidence-based practice involved?

\section{Conceptual and Theoretical Frameworks}

As one begins an inquiry into higher education, some frameworks emerge. There have been countless studies performed over the last 40 years (Elliott \& Healy, 2001; Tapp, Hicks, \& Stone, 2004; Tinto, 1975, 2006) about higher education, and how universities can be successful in recruiting and retaining students. In this section, the researcher will examine a number of these theories, both those that have traditionally be used as well as argue for a new approach or combination of approaches to examine the 
idea of organizational learning within enrollment management. The researcher will present a brief background on alternate theories and explain why those were not a good fit in this inquiry. Additionally, examined is a new approach with an argument made for its use in this research.

\section{Retention Theory}

When undertaking any inquiry into higher education, retention tends to be at the forefront of the investigation. Much research exists on college retention (Brotherton, 2001; Cabrera, Nora, \& Castaneda, 1993; Davidson \& Wilson, 2013; Elliott \& Healy, 2001; Turner \& Thompson, 2014), and the vast majority of it stems from the retention theory proposed by Tinto (1987). Retention theory is mainly focused on the individual characteristics of students and what they bring to the table (Tinto, 1987, 2006). One of the main reasons the researcher did not utilize retention theory as the lens through which this inquiry will be viewed is that it focuses on the student factors and not the institutional factors (DeShields Jr, Kara, \& Kaynak, 2005; Lotkowski, Robbins, \& Noeth, 2004). This research is focused on how organizations can learn and change to better recruit students that retain and complete college, and thus not an appropriate focus for the retention theory usage.

\section{College Choice Model}

A second perspective that is often used when researching higher education, specifically enrollment management, is the college choice model (Hossler \& Gallagher, 1987; Jackson, 1982). The phases in both models focus on the three stages of choice, an aspirational stage, a searching stage, and a deciding phase. Paulsen (1990), in the 1980s and 1990s, continued Jackson's work and began tying its importance to enrollment and 
the declining student populations. While this model does focus on the deciding phase of the EM life cycle, unlike retention theory, it still focuses on student behavior, not organization behavior. Furthermore, as with retention theory, there is no focus on the use of quantitative data, the main reason the researcher did not utilize this model for this inquiry.

\section{Predictive Modeling}

As one delves deeper into the literature (Bontrager, 2004; DesJardins, 2002;

Hossler \& Kalsbeek, 2013) about enrollment management and strategies, one invariably comes to predictive modeling. In this context, a predictive model is a statistical model used to predict enrollment, based on information gathered on prospective students and students that enroll (DesJardins, Dundar, \& Hendel, 1999) and has its roots in the fields of economics, business, and finance (Eskreis-Winkler, Shulman, Beal, \& Duckworth, 2014). The use of predictive modeling in education was first examined by Desjardins (2002) and Thomas, Dawes, and Reznik (2001). Historically, researchers in education lacked expertise in quantitative inquires (Goenner \& Pauls, 2006), which led to a lack of research concerning predictive modeling and higher education. Early uses of predictive modeling in education focused on items such as loyalty and choice (Vianden \& Barlow, 2014), but current predictive modeling is used by strategic enrollment managers to craft their incoming classes in a proactive rather than reactive way (Bontrager, 2004). This

model focuses on what the institution can do in an enrollment management office to find those students who will be successful at a specific institution. 


\section{Evidence-Based Practice}

With data and quantitative evidence established as being key to this inquiry, needed is consideration of another framework in which to examine that data. What emerged is evidence-based practice (EBP) as a bridge between quantitative and qualitative research, because it focuses on using data as a starting point and utilizing qualitative factors when making decisions (Price \& Kirkwood, 2014). EBP can be useful when holistically examining education, as qualitative data is vital in that type of examination. However, for this research, data is the focus, and EBP is too broad for the purpose undertaken here (Marsh \& Farrell, 2015). Specifically, EBP is too broad in the sense that the data is not the focus point, but the starting point.

\section{Data-Driven Decision-Making}

The review of the above theoretical frameworks revealed that a quantitative analysis was needed, and led the researcher to the conceptual framework of data-driven decision-making (DDDM). DDDM within education stems mainly from work done by Popham in the 1980s, focusing on the K-12 system (Popham, 1987; Popham, Cruse, Rankin, Sandifer, \& Williams, 1985). Historically, used sparingly in education was quantitative data, with standardized tests being the primary indicator. However, with the No Child Left Behind Act (NCLB, 2001) of the early 2000s and the international race to measure who is educating children the best, quantitative data became a known commodity in education research (Fuller, Wright, Gesicki, \& Kang, 2007). Additionally, funding became associated with being able to demonstrate an education system was working, necessitating the ability to quickly and accurately prove success (Hanushek \& Lindseth, 2009; Miao, 2012). Consequently, while used within the business or private 
enterprise world, education has been slower to adopt the use of DDDM statistics and data.

\section{Organization Learning Theory}

As discussed previously, the researcher was interested in how an organization can learn and change to use these new types of data-driven analyses. This line of inquiry led the researcher to organizational learning theory (OLT). Marsh, Pane, and Hamilton (2006) made the connection between DDDM and OLT. These researchers discussed how there are various types of data, and the job of a practitioner of DDDM is to take the various types of data and turn them into actionable knowledge (Marsh et al., 2006). The ability to turn the massive amount of data that an EM office receives into actionable knowledge is a major reason DDDM became a conceptual framework for this inquiry.

OLT primarily originates from two works; both set in a business/military setting (Cangelosi \& Dill, 1965; R. L. Chapman et al., 1959). Neither of these studies focused on education, and little research exists that discusses organizational learning in a higher education setting. OLT has been used by the business world for many years, beginning when Cyert and March (1963) outlined its critical role in the 1960s. One of the main reasons for using OLT is that research has shown that learning organizations are more competitive than those that do not embrace the idea (Chiva, 2017; Coopey, 1995; Tsang, 1997). Therefore, these reasons demonstrate why organizational learning theory will be the conceptual framework through which this research is focused. OLT creates an understanding of how an organization can learn internally and change to adopt a datadriven model of work through the research, interview, and focus group questions. This research was not interested in organizational change that happens due to a directive or a 
large influx of funding, but in a more organic change that happens as the organization learns to adapt to a model of accepted best practices in enrollment management (Langston \& Loreto, 2017; Stanton, Black, Dhaliwal, \& Hutchinson, 2017).

\section{Design of the Study}

To investigate how EM offices within two universities of varying demographics utilized OLT and DDDM to inform their practices, a parallel case study approach was selected for the design of the study. A parallel case study provides a broader detailed investigation of programs or processes and is also bound by time (Creswell, 2014). The research lends itself well to this approach as well as utilizing grounded theory, which uses the investigator(s) as the primary instrument of data collection analyzing a process or action from the view of the participants to form a general theory (Creswell, 2014). Data in grounded theory tends to come from interviews (Merriam \& Tisdell, 2016), which along with focus groups, will be the primary instruments of data gathering in this research. Interviews as a data-gathering tool are used primarily because grounded theory focuses on the view of the participants, and interviews are the stories of people, or for this research study, the participants (Seidman, 2013). While utilizing stories may not seem academic, Bertaux (1981) argued that utilizing interviews in social science research is vital as the subjects in social science are people with the ability to think and talk. These are key characteristics of subjects in social science and key differences between the subjects of social and natural or physical science.

The researcher also conducted focus groups since a focus group. This is a valuable method of collecting data in a qualitative study due to its nature of being a socially constructed group of people with knowledge in the area being studied (Merriam 
\& Tisdell, 2016). As EM offices consist of a wide variety of employees, focus groups were deemed an important way to gather data so all voices can be heard. Furthermore, focus groups give people an opportunity to discuss things in an open, safe environment, which leads to honest and truthful data emerging (Krueger \& Casey, 2015). Moreover, this research was taken before the University of Missouri Institutional Review Board (IRB) to ensure professional and ethical standards are being upheld. IRBs exist on campuses for the enforcement of federal regulations that protect against human rights violations (Creswell, 2014). The researcher included all measures to ensure the safety of all participants in the study as outlined by the IRB (see Appendix A). Appendix A also includes the letter of acceptance from the University of Missouri IRB. After permission from the University of Missouri IRB was received, a gatekeeper permission letter (See Appendix A) was sent to the Vice Provost of Enrollment Management at each institution to gain access to the study participants.

\section{Setting}

The multi-case study consisted of two universities of different types to gain a broader perspective (Hancock \& Algozzine, 2015; G. Thomas, 2015). The Universities included a large, Division 1, Research 1 public university, and a mid-size private university. The sizes and descriptions come from the Carnegie Classification System (Research, 2017).

The first university was a large, public, high research/research one university in the southern United States. The size definition of a large university and the high research definition come from the Carnegie Classification System (Research, 2017), with large being 10,000 or more students. Current enrollment at the large institution included in this 
research is 37,010 (Facts, 2018). High Research/Research 1 University comes from the Carnegie Classification System, with 1 being a university with the Highest Research Activity. There are less than 120 of these in the country (Research, 2017).

The university enrolls 37,010 students per year through a combination of undergraduate, graduate, law school, medical school, and nursing school (Facts, 2018). The university is diverse, with Hispanic students representing $27.8 \%$ of the undergraduate class, which qualifies the institution as a Hispanic Serving Institution (Facts, 2018; HACU, 2017). The undergraduate population accounts for 30,737, and the undergraduate enrollment office will be the focus of this research. The undergraduate enrollment office employs 55 full-time staff in recruiting, processing, and through the visitors center. The researcher identified the institution, through professional networking, as a university that has created their predictive model and has undergone significant change within the office of EM within the last ten years. This university will be an appropriate setting to investigate how the practices examined in this investigation progress from ideas to implementation since their senior leadership has been in place for roughly ten years. The current leadership was instrumental in the creation of their predictive model, and they fully subscribe to the ideas within organizational learning. However, a limitation to this setting is the institution resides in a sate (one of only two states) that is currently growing in high school, graduating students (Hussar \& Bailey, 2016) and one where money is less of an issue. Therefore, some of the processes they have implemented may not be replicable at smaller institutions. However, the knowledge gained from their experiences makes the inquiry valuable. 
The second institution was a mid-size, private university in the upper mid-west, in a large urban center. Mid-size, or medium university, is defined by the Carnegie Classification System (Research, 2017), with the medium being 3,000 - 9,999. Current enrollment at the medium university is 7,266 through a combination of undergraduate, graduate, and law students. Undergraduate students make up 2,724 of the total population, and the undergraduate admissions office will be the focus of this inquiry. This campus is also diverse, specifically when it comes to international students. International students make up $21 \%$ of the undergraduate population, within 100 different countries represented. This institution is a high achieving technical school, with a heavy focus on engineering, science, and architecture, with an average incoming ACT of nearly 29. The undergraduate admissions office employs 22 full-time staff members through a combination of recruiting and processing. Similar to the other case study, through professional networking, the researcher identified the institution as one that has created a predictive model and has undergone change within the last five years. This university will be an appropriate setting to investigate how the practices examined in this investigation progress from ideas to implementation, as the senior leadership has been in place for roughly five years. The current leadership was instrumental in the creation of the predictive model and fully subscribed to the ideas within organizational learning.

Private schools have a history of being early adopters when it comes to strategic EM practices (Bontrager, 2004), making the perspective gained from this institution valuable. These types of schools are usually enrolling classes of less than five hundred, sometimes less than one hundred. To be successful, they should know as much as possible about their students and their likelihood to enroll. This type of institution and the 
knowledge gained from it is valuable, as large schools have to adapt to using this private school model of recruitment, on a larger scale. As such, the private schools have an advantage over public schools, as these universities have been utilizing these strategies for most of their existence (Han, 2014). Conversely, in general, these universities lack the scalability quantity gained from investigating larger schools; however, with this being a larger private school, scalability issues will be minimal.

\section{Interview Protocol}

Interviewed was eight participants at each institution and included the Director of Admission, the Vice President/Provost for EM, a mid-level assistant director, and an admissions representative. The interviews consisted of 10 questions, each focused on a specific element of the research questions. Questions guided the subjects on explaining how their institution used organizational learning to improve their ability to recruit and retain students. Questions also focused on the data-driven decision-making model and how that affects enrollment (Creswell, 2014) (see Appendix B).

Furthermore, as Merriam and Tisdell (2016) discussed, the interview questions were established based on the research questions and determining the best way to organically gain answers to those questions. Since the researcher is an enrollment management professional, questions were piloted amongst internal EM personnel and adjusted accordingly. Questions were asked, answers were noted, and the information gained was documented. Questions were modified to allow more answers that are openended as well as ensuring all research questions were addressed in the interviews.

As outlined by Merriam and Tisdell (2016), the interview was semi-structured, where the questions will be worded flexibly, leading to a more organic conversation. 
Specific information was targeted, but rather than asking more direct and structured questions; this approach allowed the researcher to respond to the situation and to any new ideas or relevant topics that emerge naturally from the situation.

Each participant was purposefully selected as they reflect the average person represented in an enrollment management office (Merriam \& Tisdell, 2016) and to gain information on specific research questions. The director of admission was chosen to provide overall guidance on the change process, as she was the direct change agent. Additionally, this person was chosen to provide insight into the large-scale workings of the office and how leadership directly affects organizational learning. The vice president/provost for EM was included to provide additional insight into overall effects on the office, as well as how the learning done by the admissions office has affected the university on a larger scale. The assistant director and admissions representative were chosen to provide extra insight into the leadership aspects of organizational learning, as well as the day-to-day ramifications of the change. These interviews were audiorecorded, and before beginning the audio recording, each participant read an informed consent form that has been approved by the University of Missouri IRB. As participants are anonymous, signed informed consent forms were not required (See Appendix A).

\section{Focus Group Protocol}

For the focus groups, two were conducted at each institution for a total of four focus groups. One included a mix of enrollment management staff not included in the interviews, and one will consist of current students. This will allow full perspective of the enrollment cycle, from both the institution side as well as the student being recruited side. Focus groups were between five and eight participants, as this is the ideal size for 
qualitative research (Krueger \& Casey, 2015). This allows all participants to share their viewpoints and feel equally included.

To ensure validity (Creswell, 2014), questions for the focus group were created using the researchers own background as an enrollment management professional and tested for validity. Questions were pilot-tested internally within an enrollment management office, both with professional staff and current student workers, to ensure they were understandable and provided answers to the relevant research questions and topics (Krueger \& Casey, 2015). Similarly to the interview questions, focus group questions will be semi-structured, as outlined by Merriam and Tisdell (2016), meaning the questions will be worded flexibly, leading to a more robust conversation (see Appendix B).

The focus group questions with the employees will pertain to the perceived changes and successes, as discussed by those using the new models created by the senior administration. The employees were selected purposefully (Krueger \& Casey, 2015), as discussed previously. The focus group with the students discussed how this universities recruitment differed from others that the students interacted with, and what about this model made them choose that school (Creswell, 2014). Students were selected via organizational recruiting (Krueger \& Casey, 2015) meaning that volunteers from the current student body were accepted. In addition to the random student volunteers, current student workers within the admissions office also volunteered and were randomly selected from the pool of volunteers to join the focus groups as well. These focus group sessions were audio-recorded, and before beginning the audio recording, each participant read an informed consent form that had been approved by the University of Missouri 
IRB. As participants are anonymous, a signed informed consent form was not necessary (See Appendix A).

\section{Document and Artifacts}

Documents and artifacts are key components in the data collection efforts of qualitative research (Merriam \& Tisdell, 2016). In general, documents are materials, while artifacts are things or objects that exist in the environment of the study (Merriam \& Tisdell, 2016). An important category of documents used in this study was records and statistical databases. In the field of education, public documents such as statistics and data are vital to telling the whole story, and specifically, things not observed (Merriam \& Tisdell, 2016). Specific data related to this study was currently not publicly available, which makes its inclusions vital. For this study, the researcher used data collected by the National Student Clearinghouse (NSC) as well as the Integrated Postsecondary Education Data System (IPEDS). This data set included specific information on the number of students who persist and graduate at both institutions and comparative data on a national level. Specific documents were not be included as much as data from the sources mentioned above. The purpose of adding this national data was to validate enrollment data as presented by the university. The overriding theme of this research was how an enrollment office can be successful based on student growth. Data triangulation with internal, institutional data along with the national data assisted with validity (Creswell, 2014).

\section{Data Analysis Procedures}

Data analysis within qualitative research is both ongoing and all-encompassing (Creswell, 2014). The researcher should analyze data throughout the study, as opposed to 
gathering all the data and then analyzing at the end, as in quantitative research.

Additionally, the analysis must consider all aspects of the research and the interactions amongst the various parts. In other words, that data does not live independently but is intertwined (Creswell, 2014). Furthermore, when utilizing a case study, the researcher must pay strict attention to data management, as multiple sources of sometimescontradictory data can lead to confusion and misinterpretation (Merriam \& Tisdell, 2016). Merriam and Tisdell (2016) similarly stated that to begin the data analysis in a case study, and all the evidence must be brought together to be analyzed, both as a whole and as independent parts.

The researcher conducted continuous data analysis throughout the study to triangulate the data collected. After the transcription of the interviews and focus groups, in which the researcher used member-checking (Creswell, 2014) taking the final report and themes gathered from the research to the participants to see if they feel it accurately represented what they were trying to express) to determine the accuracy of the findings, the researcher read the transcripts in their entirety to obtain a broad perspective of the participants' perception and code for themes (Merriam \& Tisdell, 2016). Coding here means organizing the data into segments that correlate to themes as they relate to the research questions (Creswell, 2014). Practices include but are not limited to segmenting sections of text from interviews and focus groups, highlighting, creating theme categories, and creating theme terms that correspond to research questions (Creswell, 2014). The researcher specifically examined topics that are based on past literature and experience, as well as topics that were not anticipated at the outset of the research. Once initial codes are created for topics, codes can then be grouped in a process known as axial 
coding (Merriam \& Tisdell, 2016). This coding is useful in that it goes beyond simple topic grouping and includes grouping based on meaning and thematic consistency.

\section{Limitations and Assumptions}

The major limitation of this study was the lack of accessibility to all students who could participate in the focus groups. The most effective way to gain insight into what practices of enrollment management impact a student's decision to enroll at a university would be to directly interview the students. Hence, the entire purpose of interviews (Creswell, 2014). However, due to the difficulty of gaining access to current students, this study used a mix of current students and student workers in the admissions offices of both institutions. While this student perspective is valuable, most of these students will not be recently admitted, and they carried potential bias due to their employment within the offices of admissions. For a more inclusive focus group, participants would be from the general student population, and all would have been within one semester of being admitted. Therefore, all students interviewed would be recently recruited to the university.

In an ideal environment, this research would have included participation from a mid-sized, regional, public school, to include a more robust sampling of university types. Public institutions in the Midwest are encountering record budget cuts, drops in enrollment, and challenges from all sides as to their value (Calderon \& Mathies, 2013). These schools represent a large percentage of universities in the mid-west, and their perspective would be valuable. However, gaining access to these schools is difficult, as universities in the Midwest have strong competition with other, mid-west, regional, public institutions. 
A final limitation to this study included the researcher's connection to the universities and the leaders interviewed. The primary change agent and leader at both schools were former employers of the researcher, and the researcher worked for nearly seven years at the large university. Potential bias has framed the approach to the research, and consequentially, all data from interviews, focus groups, and document analysis was vetted using member checking to ensure that personal bias does not affect the transcription of the sessions (Creswell, 2014).

\section{Design Controls}

The researcher utilized several controls to reduce bias in the research. Initially, the researcher used only semi-structured open-ended questions in the interview and focus group settings. This allows the participants to share opinions and views without being influenced by the researcher's viewpoints (Merriam \& Tisdell, 2016). Coding of the data was done in a manner that is consistent with current practice and the established literature in the fields of higher education, enrollment management, data-driven decision-making, and organizational learning, including the use of axial coding (Creswell, 2014). To this end, member checking allows bias to minimize by participants reviewing their responses and making changes to convey the correct information and tone (Creswell, 2014). While the researcher does have a personal connection to the leaders at the two institutions being examined, random sampling of focus group participants and interview participants (when possible), reduced the potential of bias.

\section{Definition of Key Terms}

Key terms defined help provide an understanding of the research and its key components. Thus, described are the following terms that guided in this inquiry: 
Enrollment Management: there have been many definitions of enrollment management (EM) over the years of research, with the first coming from Kemerer, Baldridge, and Green (1982). The researchers defined EM as "An assertive approach to ensuring the steady supply of qualified students required to maintain institutional vitality (Kemerer et al., 1982, p. 3)." A slightly more detailed definition will be the primary one used when discussing EM for this study. This definition from Bean and Hossler (1990, p. 300) noted EM is an "organizational concept and systematic set of activities whose purpose is to exert influence over student enrollments."

Four years, large university: The size definitions come from the Carnegie Classification System (Research, 2017), with large being 10,000 or more students. Current enrollment at the large institution included in this research is 37,010 (Facts, 2018).

Four years, medium university: This size definition comes from the Carnegie Classification System (Research, 2017), with the medium being 3,000 - 9,999. Current enrollment at the medium university included in this research is 7,266.

Research 1/High Research University: This definition comes from the Carnegie Classification System, with 1 being a university with the "Highest Research Activity." There are less than 120 of these in the country (Research, 2017).

\section{Significance of the Study}

As stated in the introduction, the number of students who are attending college is declining, and to remain solvent, institutions of higher education must adapt and learn (Hossler \& Kalsbeek, 2013). This research focused on helping institutions, with the end goal of creating a predictive model that will allow them to recruit more effectively and 
efficiently students who will successfully retain and graduate. This is important because if schools can reach students more effectively, the likelihood of successful completion increases (Brotherton, 2001; Kuh, Kinzie, Schuh, \& Whitt, 2011). If students do not select the right college, the school where they have the best chance of being successful, these students are less likely to graduate (Ferris, Finster, \& McDonald, 2004; Mattern, Shaw, \& Kobrin, 2010). This leads to fewer people with college degrees, more loan debt for students, and wasted time for all involved (DesJardins et al., 2006). For those wanting to attend an institution, all parties are best served by a model that gets students to the right place in the most efficient manner (Tucker \& McKnight, 2017). The institutions benefit because they can grow enrollment, increase retention and graduation, and utilize resources elsewhere to help the institution (Poole, Levin, \& Elam, 2017). Students benefit by not wasting time with an extended search process or spending time and money with transferring between institutions. It is important for students to find the right fit for themselves, which will help them graduate in a more efficient and cost-effective time frame (Witteveen \& Attewell, 2017).

Additionally, this research can lead to further inquiries into what makes for successful EM practices, but further, more direct research into organizational learning. Organizational learning is going to become vital to the sustainability of all businesses, not just higher education (Lozano, 2014). If organizations cannot learn to adjust internally, these institutions will not be able to survive, particularly as it applies to higher education.

\section{Summary}

In summation, a case study design was undertaken, utilizing focus groups, interviews, and document analysis to investigate how departments of enrollment 
management have used organizational learning and data-driven decision-making to inform their enrollment practices. The initial focus was replicable best practices from other institutions of higher education. Additionally, research examined how EM offices created a predictive model for future enrollment. There is little research on this topic as a cohesive unit. Current research (Kabakchieva, 2013; Le, Robbins, \& Westrick, 2014) focuses only on specific practices being studies. For example, there is a large volume of research on organizational learning and data-driven decision-making in the business world (Provost \& Fawcett, 2013; Real, Roldán, \& Leal, 2014) and K-12 education (Erdem \& Ucar, 2013; Kaufman, Graham, Picciano, Popham, \& Wiley, 2014), but little involving higher education.

Within enrollment management, there is plenty of research on predictive modeling and best practices (Antons \& Maltz, 2006; Cabrera et al., 1993; Goldstein \& Katz, 2005), but minimal research on replicable efforts without large grants or other funding. This research was important due to the changing landscape of enrollment within higher education, and institutions should place on student enrollment, retention, and graduation. Universities personnel should be willing, and more importantly, can change rapidly. Moreover, personnel should be able to identify needed change. This research attempted to answer the question of how to achieve that knowledge, as well as provide a foundation for further research into organizational learning within higher education. 
SECTION TWO

PRACTITIONER SETTING FOR THE STUDY 


\section{Introduction}

Presented within this section, were the history of the two universities as well as their demographics, the organizational structure of the schools, and specifically the enrollment management departments. Furthermore, presented was the vision and the mission of the universities. Additionally, there was an analysis of the leadership within the universities and implications of this research for these universities, and other, similar schools.

\section{History of the Organizations}

\section{Large Public University}

Large Public University (LPU) is a large, public research university with the main campus located in the southern U.S. The LPU campus compriseses a major research university, a medical school, and a law school. The school, opened its doors in 1925, with four schools and an enrollment of 914. In 1969, by the action of the State Legislature, the school officially became LPU. In 1996, the LPU system was created, encompassing the main campus, the medical school, and the law school. Over the years, this system has grown to include many other off campus locations and specialty schools. Today the university has ten colleges and two schools on the main campus and an enrollment approaching 38,000. LPU is considered a large, public university with the highest research activity possible (Research, 2017).

The students that makeup LPU are a diverse blend of in state and out-of-state as well as many international students (9\%). Additionally, LPU is represented by a wide range of ethnicities, including 27.8\% Hispanic students, $6 \%$ African-American, and 55\% Caucasian. With the growing Hispanic population in the southern U.S., LPU has become 
a Hispanic Serving Institution, one of 250 in the country, gaining this designation from the Department of Education and receiving grant money. To receive this designation, along with some other criteria, universities should have at least $25 \%$ of their undergraduate, full-time enrollment be Hispanic students (Education, 2008).

The initial focus of the university was on technological areas. However, the school's focus expanded and now offers over 150 undergraduate degree programs in everything ranging from art and theatre to business and engineering. The College of Engineering is a top 100 engineering school in the nation (Cunningham, 2014). With the expansion to a university system, the focus on the university has grown, and the LPU System has produced over 200,000 alums since the first graduating class of 1927.

\section{Medium Private University}

Medium Private University (MPU) is a medium-sized, private, doctoral-granting, Research University in the United States midwest, founded in 1940. The focus of MPU is technology-based research and has several locations across the United States, including five campuses in the Chicago area.

The students that makeup MPU are a diverse group, especially when it comes to regions of the world served. MPU has a total enrollment of 7,266, made up of undergraduates, masters students, doctoral students, and law students. Fifteen percent of MPU students are under-represented minorities, and 36\% are female. Additionally, 21\% of undergraduates and $62 \%$ of graduate students are international students, and $60 \%$ of undergraduates are in-state, with $40 \%$ being out of state. This represents a significant shift in the demographics of the campus when approximately three to five years ago, a concentrated effort was made by enrollment management at MPU to reduce dependency 
on in-state students (maxing out at $83 \%$ ). Currently, MPU is mainly focused on STEM fields, science, technology, engineering, and math, as well as having a robust architectural program.

\section{Organizational Analysis}

A ten-person Board of Regents oversees the LPU system and meets approximately every other month, depending on the year. The board oversees the chancellor, who is over the main LPU campus as well as all offsite locations, all part of the LPU System. For this research, the focus is the main undergraduate campus and overseen by the president, who reports directly to the chancellor. The president has nine direct reports who make up the leadership team that guides the day-to-day operations of the university. Overseeing enrollment management is an assistant vice president for enrollment management, followed by an executive director and a managing director, followed by numerous assistant directors and staff. At LPU, the executive director has been in the office the longest and has been the major change agent, implementing the transition to data driven decision making in her ten years of leading the office.

Similarly, an eight-person Board of Trustees oversees MPU, and they directly oversee the president. As they are not a system, they do not have a Chancellor. The president directly oversees the vice president of enrollment, who oversees the assistant vice president of admission. This is a recent change at MPU, as enrollment management, before a year ago, reported to the provost. The provost, who has a background as an engineering professor, and was heavily involved in enrollment management before becoming the provost, recently became the President of MPU. Due to the strong 
relationship between enrollment management and the former provost, the structure changed to have enrollment management report directly to the president.

Additionally, the former provost, now president brought in a new VP of enrollment, who has a background in enrollment management consulting and is very data driven. The vice president of enrollment distributes a great deal of data and reports directly to the president. When researching the background of MPU, the researcher spoke with the AVP of Admission, and she indicated that the VP, her direct supervisor, is excellent with data, that is her background, and focuses her time on data while allowing the AVP to lead the admissions office (Riley, 2018).

At both organizations, there are clear connections to the structural and human resource frames, as discussed by Bolman and Deal (2013), and it is through these two frames that the two organizations will be analyzed. Overall, Bolman and Deal (2013) stated, a frame is nothing more than an idea or set of ideas that allows one more opportunities to see and better understand an organization. The structural frame examines an organization from its functions and reporting levels. Higher education is structured and reliant upon organizational charts and the distribution of labor. The structural frame provides a rational way to approach this analysis.

According to Bolman and Deal (2013), the structural frame is comprised of six assumptions, a significant assumption for this study is specialization, and division of labor increases efficiency by design. The idea of specialization increasing efficiency started in the early $20^{\text {th }}$ century and included some of the original theories that influenced Bolman and Deal's structural frame. A key study edited and contributed by Gulick and Urwick (1937) focused on what they called organization theory. The authors argued 
people differ in skill, and people cannot do two things at once or be in two places at once. Thus, each person possesses a small part of the vast range of knowledge that exists, specialization and division of labor makes effective business sense. Weber (1947) expanded on this work, especially about the idea of a fixed division of labor. In his work, Weber discussed the purposes of the bureaucratic organization and discipline, studying businesses, political organizations, armies, schools, hospitals, and various other structured organizations, coming to the conclusion that division of labor is vital to making a formal organization work as efficiently as possible (Weber, 1947).

In the case of LPU, the structure of the admissions office appears radically different than it did ten years ago when current leadership took over. There are three main areas in their admissions office, the visitors center, recruitment, and processing. Historically, these three areas had been segmented and isolated. Current leadership made it a priority to build a cohesive team environment, which still allowed for specialization of work, but revealed every employee is valuable to each other. For example, processing and recruitment traditionally have minimal contact. To processors, the employees that manage the enormous amounts of paperwork (transcripts, test scores, letter of recommendation, etc. ) that an admissions office receives, recruiters were viewed as fresh college graduates who travel all the time and had fun on the road. To the recruiters, the processors were regarded as stodgy older people who could not keep up with scanning a few documents and never had any fun. One of the priorities of the new leader ten years ago was to show these two areas the value of the other. Therefore, she had the recruiters work for a week staying in the office, processing and had processors take a week on the road with a recruiter. The value and difficulty of each person's job quickly became 
apparent to the other side, and a much better working relationship was established. By breaking down barriers and restructuring the organization, better results were achieved. As Bolman and Deal (2013) stated, "troubles arise and performance suffers from structural deficits, remedied through problem-solving and restructuring (Bolman \& Deal, 2013, p. 45)."

At MPU, a different change in the structure of the organization yielded similar results. Bolman and Deal (2013) discussed the value of specialization and division of labor by noting how in the structural frame it is essential to design the organization to properly allocate work as well as to coordinate various efforts amongst the group. MPU recently modified the structure of their admissions representatives or recruiters as an example. As mentioned above, MPU is a very technical massive school, with a strong focus on STEM fields. One of the things that leadership observed was that the types of students recruited sometimes had a difficult time making connections early in their college careers. Recruiting is typically done territorially; a recruiter will oversee a certain territory, a state, region, or part of a large city such as Chicago. At MPU, they recently altered the role of admissions representatives to include what they call passion projects. This allows recruiters to oversee their territory and additionally choose to focus on an area or student population. Therefore, recruiter X may have the south side of Chicago as well as all first-generation college (FGC) students, meaning they recruit the south side, but they work with and oversee a student group comprising of FGC students once enrolled on campus. This serves the dual purpose of allowing recruiters to have a personal buy-in to students once they are enrolled and attending campus and providing the incoming students with a built-in support group. 
Additionally, this minimizes some of the inherent competition issues that arise in an admissions office. People who work in admissions tend to be competitive, and while that can be positive, sometimes cooperation and success can be limited by competition (Levi, 2014). The current model allows recruiters to work together collaboratively, because one recruiter may not be directly recruiting the students who will be in his passion project group upon enrollment. MPU has seen great success with this model and plans to continue to expand its reach.

\section{Leadership Analysis}

At both institutions, the leadership is responsible for the shift to data-driven decision making. At LPU, the leadership focus will be the current executive director. The Executive Director has been with the LPU Office of Undergraduate Admissions for a little over ten years. Before that, she spent two years in the LPU Advising Center and previously spent nearly five years in admissions at a midwestern state university. When she took over, the admissions office did not effectively use data, and the areas under her were significantly isolated. She needed to change the culture (Levi, 2014) and made that a top priority. At the time she took over and in the years prior, LPU was seen as the state school you went to if you could not get into one of the more academically prestigious schools in the state. Senior leadership at LPU wanted to change that image, as well as work towards the university becoming a Tier 1 Research University. This is the highest ranking a research university can achieve. A significant part of becoming a Tier 1 Research university pertains to the incoming class. For example, more than $25 \%$ of the incoming class must be in the top $10 \%$ of their high school graduating class. 
Additionally, universities must bring in at least $\$ 100$ million in research money each year (Research, 2017).

Furthermore, the leadership wanted the university to increase enrollment, achieving 40,000 total students by the year 2020. Confronted with this challenge, growth in size while also raising quality, the executive director took immediate steps to revolutionize the admissions office. As discussed previously, she removed barriers that had plagued the unit, creating cooperation among teams of recruiters, visitors center staff, and processing (Levi, 2014). They engaged in strategic name buying, strategic, datadriven recruitment planning, and the process of creating a predictive model. They revamped the entire communication flow and created multiple collaborations across campus that had not existed before. She and her team became engaged at the state and national level, helping to lead efforts strategically by being at the table when decisions were made (Northouse, 2016). Nearly all aspects of day-to-day activity at LPU have changed in the last ten years, and the results are positive. They are now on their $12^{\text {th }}$ consecutive semester of record enrollment, and their quality continues to grow with LPU reaching Tier 1 Research University status in 2016 and are on pace to achieve the enrollment goal of 40,000 by 2020 .

At MPU, the leadership team consists of an assistant vice president of admissions, who has been there for nearly five years and who spent over ten years working at MPU, most of them under the executive director mentioned previously. There is also the VP of enrollment discussed earlier, with the background in consulting. At MPU, they have formed a management team (Levi, 2014) that shares the responsibilities for the organization. The VP brought about the shift to data-driven decision making due to her 
background as a consultant and her desire not to be involved in the day-to-day minutiae of the admissions office. Before her arrival, data was not utilized to shape the class, and as a result, MPU was reliant on in-state students, similar to a regional public school.

Futhermore, most of their students were on the low end of the acceptable academic criteria, and low income that was causing retention issues. As a private school, the campus leadership wanted them to have a broader recruitment strategy, both in the United States and internationally, as well as raise their academic profile. Under the leadership of the VP and the AVP, MPU started strategic name-buying, recruitment for different scholarship programs, involved alums, and planned recruitment travel based on data and the strategies mentioned. Results have been immediate and successful, with the quality of the class raising both academically and in the scope of their representation, along with greater involvement from alums and other partners.

In both case studies, the leaders involvement were both transformational and authentic (Northouse, 2016). Transformational leaders are leaders who change people and organizations with whom they are involved. There are aspects of charisma and influence with transformational leaders. Transformational leaders have a lasting and measurable impact on people and organizations (Northouse, 2016). Another interesting way to examine this is through the idea of the person-environment conceptualization model of transformational leadership (Lambert, Tepper, Carr, Holt, \& Barelka, 2012). In this model, the authors argue that the real value of transformational leadership comes from the followers satisfying their psychological needs of workplace stability, varying work experiences, and need activation and satiation. Moreover, transformational leadership is the value in how the followers are changed or fulfilled. The unusual use of this theory, as 
further examined in a recent study by Tepper et al. (2018) is to investigate if the leaders are focused on their followers and fulfilling their needs.

Authentic leadership focuses on the realness or authenticity of a leader. Authentic leaders are seen as genuine, trustworthy, caring, and committed (Northouse, 2016). It is important to note that this does not mean authentic leaders cannot be confrontational or disagree with team members or are nice all the time. It is more of a philosophy of what you see is what you get. Authentic leaders are real, and you should always know where you stand with an authentic leader. To look at this from a follower perspective, multiple recent studies (Leroy, Anseel, Gardner, \& Sels, 2015; Rahimnia \& Sharifirad, 2015; Weiss, Razinskas, Backmann, \& Hoegl, 2018) have revealed authentic leaders help followers to fulfill their basic needs in a work environment. Also, authentic leaders have an increase in output, overall happiness, satisfaction, and mental wellbeing, and the strength of their relationships.

\section{Implications for Research in the Practitioner Setting}

With the ever-growing pressure on enrollment managers to produce growth with quality in their incoming classes, organizations must be able to learn and change. Datadriven decision-making is one strategy that has proven effective in helping enrollment managers be successful. With the knowledge gained from this research and other inquiries into EM success, EM practitioners can begin to create a toolkit for success, while simultaneously helping their organizations change and adapt to today's market. Reviewing the literature, as well as the data findings, will allow enrollment managers to gain a better understanding of their changing profession. Additionally, similar research can help EM leaders understand the why behind the strategies as well as the strategies 
themselves. Finally, this investigation and the relevant literature show that a strong team is necessary, as the only way for an organization to learn and change. People in the organization must be willing and able to learn and change. It is the job of a transformational leader to help their employees, and their organization accomplishes that change. Furthermore, authentic leadership is especially useful in situations of organizational learning and change, as authentic leaders are in tune with their employees and garner a great deal of trust, which is critical for any change efforts.

\section{Summary}

This section provided a brief history of both LPU and MPU. Provided was an analysis of both organizations, utilizing the established structure of the admissions offices of both schools, as well as a basic introduction to the leadership structure. The analysis highlighted recent and past developments that have led to the increased use of data-driven decision-making and organizational learning that has taken place at both institutions, and the resulting success. Furthermore, this section provided leadership analysis on the key leaders at both institutions and how their style of leadership facilities organizational learning, as discussed throughout this research. In conclusion, theorized were the implications of this research as to the impact this research could have on other admissions and EM offices. 
SECTION THREE

SCHOLARLY REVIEW FOR THE STUDY 


\section{Introduction to the Scholarly Review}

Estimates reveal the number of high schools graduating students are declining in the United States in all but two states, Florida and Texas (Hussar \& Bailey, 2016). This highlights that offices of Enrollment Management (EM) within institutions of higher education should learn how to be more effective and efficient when recruiting students. In reviewing the current research on this topic, trends begin to emerge quickly. There is a great deal of literature on national best practices within enrollment management, and how enrollment management is similar to marketing (Berry, 1995; Bontrager, 2004; Hossler \& Kalsbeek, 2013; Payne \& Frow, 2005). Similarly, how to integrate enrollment management to serve the university effectively has been investigated (Bowen, Bok, \& Burkhart, 1998; DesJardins et al., 2006; DesJardins et al., 1999; Hossler \& Kalsbeek, 2013). This research all focuses on either the role of a successful EM office or the strategies that successful EM offices use for decision-making. Conversely, there is a lack of research on how an EM office becomes such an entity that can effectively use these strategies.

It is from this gap in the literature review, whereby this researcher is seeking to examine how an EM office can become effective in utilizing all the strategies the existing research discusses. The focus of this examination are two institutions that have changed at the fundamental level of enrollment management, with little to no outside funding, and have created a predictive model of recruitment and an overall model of sustainability about successful enrollment management. Specifically, this review of literature will provide an understanding of the constructs involved, present evidence of their connectivity, and provide a conceptual basis for the investigation. 
There are some potential lenses in which to view this problem, most notably retention theory, as put forth by Tinto (1987) in the 1980s. Other approaches initially examined were the College Choice model (Paulsen, 1990), Predictive Enrollment Theory (DesJardins, 2002; Goenner \& Pauls, 2006), and Evidence-Based Practice (Lodge \& Matthews, 2017; Slavin, 2002).

\section{Retention Theory}

Historically much of the research on EM and admissions issues was focused through the lens of retention (Tinto, 1987), as it was both an uncomplicated way to measure success and a national statistic that was gathered by all institutions of higher education. Specifically, through this literature review, examined was retention theory as a possible theoretical framework from which to approach this research. Retention theory is based mostly on the work of Tinto, who focused on the individual characteristics of students and what they bring to the table (Tinto, 1987, 2006). Tinto's theories are the foundation of studying retention in education, and there are numerous studies using retention theory as a lens in which to examine EM (Davidson \& Wilson, 2013; Stanton et al., 2017).

Most of the recent research on Tinto's work is focused on retaining specific groups, such as racial and ethnic minorities (Museus, 2014; Strayhorn, 2014), online and distance education students (Freitas, Morgan, \& Gibson, 2015), and males (Voyer \& Voyer, 2014). As colleges and universities become more and more adept at strategic enrollment management and identifying specific populations they wish to target in the recruitment process; the focus has become more on targeted recruitment and retention, rather than admitting anyone who will attend (Berry, 1995; Bowen et al., 1998). This 
leads to the idea of crafting a class (Ehrenberg, Zhang, \& Levin, 2005), which is more about identifying an incoming class of students that have a particular set of characteristics, compared to allowing anyone possible into the university. While the research agreed this is an essential aspect of strategic enrollment management (Bontrager, 2004; Hossler \& Kalsbeek, 2013), when viewed through the lens of retention theory, the focus is students that possess specific characteristics and, not on how the organization can become one that can identify those characteristics.

Furthermore, current research focused on retention theory is primarily concerned with the millennial generation of students and their specific needs, not the organization (Turner \& Thompson, 2014). Moreover, the research regarding millennials and retention focuses on the parent-student relationship (Barton \& Hirsch, 2016), the faculty-student relationship (Romsa, Bremer, \& Lewis, 2017), and the relationships between students and all campus services (Sidelinger, Frisby, \& Heisler, 2016). This research is important and helpful when discussing student success and retention; however, for purposes of this research, it is on student factors. While the analysis does consider interactions with the campus community, this research study will analyze student characteristics and behavior. Another idea that is often considered when looking at retention is the usefulness or success of orientation and first-year success programs (Bell, Gass, Nafziger, \& Starbuck, 2014; Permzadian \& Credé, 2016; Wibrowski, Matthews, \& Kitsantas, 2017). While these inquiries bring us closer to learning about institutional programs as opposed to student characteristics, this research is focused on the enrollment management functions of a university, not the student success functions. Whereas some enrollment offices do 
house orientation, the focus of this research is on the recruitment aspect of the enrollment cycle, not student life and retention.

As stated previously, retention theory is focused on the factors a student possesses that make him or her more or less likely to retain (Davidson \& Wilson, 2013; Tinto, 1987, 2006), rather than how an organization can learn and change to better recruit students. This research study focuses on the internal processes within organizations and how organizations can learn to predict better how a student will make their college choice. The next model examined brings us closer to that idea, as it examines the college choice model, unlike retention theory that centers on the student factors that affect retention, not initial college choice.

\section{College Choice Model}

The College Choice Model comes from various researchers who proposed the three-phase model of choice, most notably Jackson's (1982) work in the 1980s. Jackson suggested the initial three-phase model of choice, the aspiration formation stage, the college search and application phase, and finally, the selection and attendance phase. The aspirational phase is when the student begins to consider what his or her preferences are when it comes to college. While Jackson (1982) stated many factors affect preference, the three leading factors are previous academic achievement, context (peers choices, location, and type of college), and family background. The search and application phase is typified more on exclusion Jackson (1982) noted, observing there are typically a large number of colleges that fit a student's basic criteria list, so exclusions should be made on outlying factors such as the preference factors mentioned above along with the significant cost consideration. With college more expensive today than when Jackson's research was 
conducted, it could be argued that cost is an even more significant preference factor for students today. Paulsen's (1990) work on student enrollment behaviors in the 1980s and 1990s, especially as it relates to cost, continued Jackson's work and began tying its importance to enrollment and the declining student populations. Paulsen focused on the real cost of attending college and the cost-benefit ratio of a college education, which, again, with the rising cost of college only becomes more relevant for today's student (Paulsen, 1990; Paulsen \& John, 2002)

On the third and final phase, Jackson (1982) noted the evaluation or selection phase. One interesting point he made is while research is focused on this area, he argues that this state is somewhat anti-climactic, with the decision essentially being made in the other two phases, albeit often subconsciously. When a student makes a list of the essential factors and begins eliminating options, often a winner emerges, with the choice being more of a result of only one school hitting the marks, as opposed to a conscious decision.

Most of the additional work on college choice uses this three-phase model as its basis. Similarly, Hossler and Gallagher (1987) introduced their own, similar, three-phase model, exploring the role of socioeconomic status in the college choice process, something that Jackson did not consider. As mentioned above, the cost was not the issue in 1982 as it is today, so socioeconomic status and cost become more and more prevalent in the college choice research as time progresses (Skinner, 2018). Other key introductions that Hossler and Gallagher (1987) made include the specific role of parents in the choice process, the value of interactions with higher education organizations (particularly of interest in this research), and how and when higher education institutions distribute 
information to prospective students. In addition to the key insights provided by their study, Hossler and Gallagher set the stage for the next wave of research into college choice by beginning the focus on individual or special populations of prospective students.

There is a large body of research on college choice among special populations such as race and ethnicity (Manski \& Wise, 1983; Perna, 2000, 2006), family income (An \& Sorensen, 2017; Brown, Wohn, \& Ellison, 2016; John, 1990; Paulsen \& John, 2002), and parent education (Chiu et al., 2016; Kansal \& Kaur, 2016; Manski \& Wise, 1983). The current research on choice when related to race and ethnicity and family income tend to overlap. The investigation revealed both students of color and students from low socioeconomic backgrounds are less prepared for higher education that white middle and upper-class students, as well as having less access to higher-level math courses in their high schools (Adelman, 2006). Solorzano and Ornelas (2004) also noted schools with fewer resources, and those that are populated mainly by students of color offer fewer advanced placement courses. The research mentioned above and other research focused on parent education, and family income also tends to be grouped with socio-economic status. The education level of the parent is often a direct reflection of the socio-economic status of the family (Kao \& Thompson, 2003). This issue also becomes intertwined with race, as researchers have noted the increasing stratification of higher education based on race, ethnicity, and social class (Freeman, 1997).

While this model does focus on the deciding phase of the EM life cycle, there is information on student behavior, not organization behavior. Additionally, similar to retention theory, the focus here is on student factors, not organizational factors. 
Furthermore, there is no focus on data output, or the use of quantitative data, in a strategic manner (R. Chapman, 1984; Hossler \& Gallagher, 1987; Paulsen, 1990). As shown, the prevailing theories historically used to evaluate higher education focus on student factors, not organizational ones, as well as lacking a depth of focus on data. The next model to be discussed takes the discussion one-step further and introduces an analytic and historically business-driven process of data analysis called predictive modeling.

\section{Predictive Modeling}

Predictive modeling has its roots in the fields of economics, business, and finance, and has been used in all facets of life, including predicting the success rates of marriages and careers (Eskreis-Winkler et al., 2014). In recent years, predictive modeling has led the way for data mining (Rygielski, Wang, \& Yen, 2002), a similar process, but one that is focused on consumer behavior. Data mining has even made its way into education. However, similar to data mining as a consumer behavior tool, data mining in education is focused on improving the consumer (student) experience rather than predicting behaviors (Romero \& Ventura, 2013). The use of predictive modeling in education was first examined by Desjardins (2002) and Thomas, Dawes, and Reznik (2001). One primary reason for the lack of research in this area is the underlying, arguably incorrect, assumption, which institutions of higher education lack the in-house expertise to create an accurate predictive model (Goenner \& Pauls, 2006).

A predictive model in this context is a statistical model used to predict enrollment, based on information gathered on prospective students and students that enroll (DesJardins et al., 1999). Additionally, some predictive models are used in retention 
rather than enrollment function. For example, some institutions of higher education use predictive models to anticipate students that may be at risk for dropping out (Thammasiri, Delen, Meesad, \& Kasap, 2014), especially in at-risk populations (Márquez-Vera et al., 2016). In recent years, institutions of higher education have doubled down on the inhouse expertise in this area. Offices of Institutional Research, once run by educators, have begun to be taken over by economists and mathematicians, leading to a renaissance in predictive modeling in education (Langston \& Loreto, 2017). While the early use of predictive modeling focused on items such as loyalty and choice (Vianden \& Barlow, 2014), current predictive modeling is being used by strategic enrollment managers to craft their incoming classes in a proactive rather than reactive way (Bontrager, 2004). Big data and the use of predictive analytics has become pervasive in all of higher education, but nowhere more so than in EM (Daniel, 2015). The pace at which the business of education now moves and the sheer volume of data available to enrollment managers makes the use of predictive modeling not a luxury as before, but rather a necessity. However, for this research, predictive modeling does not go far enough. Predictive modeling is an excellent tool to use for an enrollment management office (Bontrager, 2004; D. Hossler \& D. Kalsbeek, 2013). However, it is not a structure in which one can organize an office, it is merely a tool. This research is focused on how an organization can implement a new style of operating to both tell its story and be successful.

\section{Evidence-Based Practice}

Evidence-Based Practice (EBP) is similar to Data-Driven Decision Making (DDDM) and is interchangeably in the literature with DDDM. The critical difference, however, is that EBP is focused on using evidence as a starting point, while still 
including qualitative factors when making decisions (Price \& Kirkwood, 2014). DDDM is focused on using data and data alone, specifically, statistical types of data. EBP is a strong model when evaluating education as a cohesive unit (Biesta, 2007). However, for this research, data is the focus, and EBP is too broad for the purpose undertaken here (Marsh \& Farrell, 2015). Like Predictive Modeling and DDDM, EBP has its roots in the business world as well as in the medical field (Isaacs, 2003). The business world has never relied on anecdotal evidence, requiring concrete and replicable statistical data, while the medical field is known for randomized studies that are purely quantitative. These types of studies are nearly impossible in the area of education, as they involve withholding a variable from one group in the study (Biesta, 2007). As education research usually consists of the administration of a new type of program, it would be considered unethical to withhold services to students (Creswell, 2014).

Education has begun to catch up in the last 50 years, and EBP was one of the first significant steps towards that goal (Biesta, 2007; Levant \& Silverstein, 2005; Slavin, 2002). In general, one of the early concerns with EBP and the use of data in education was the integrity and reliability of that data (Biesta, 2007). However, as educators and education administrators have become more adept with EBP, data use has increased dramatically. Some educators still fight against the use of EBP or DDDM within education, instead relying on their stories and experiences to prove successes (Kowalski \& Lasley, 2010).

\section{Conceptual Frameworks}

Ultimately, this review of the relevant literature led the researcher to organizational learning theory (OLT) and data-driven decision-making (DDDM) as the 
two conceptual frameworks in which to review this investigation, both discussed in detail in the following sections. The critical element of OLT that leads to its use as the conceptual framework is that the data will be focused on organic, internal change. For these new methods of EM to be successful and sustainable, they must come from within the university. It is vital that organizations learn how they can make this change happen themselves, and for that to happen, individuals must understand how organizations learn (Hoppes \& Holley, 2014).

The stated goal of this change process and the desired outcome, according to the inquiry, is for organizations to be able to create a predictive model of their own based on their data and resources. Institutions should evaluate their ability to collect data, analyze data, and then eventually make decisions based on that data if a predictive model can ever be achieved (Bouwma-Gearhart \& Collins, 2015). Additionally, regarding DDDM, institutions are being required to collect and present statistical evidence to justify funding more now than ever before (Hussar \& Bailey, 2016), particularly with the increase in performance-based funding models (Rutherford \& Rabovsky, 2014).

\section{Organizational Learning}

Organizational learning theory (OLT) primarily originates from research in the late 1950 s and early 1960 s. The first mention of organizational learning comes from a 1959 study that examined how air defense workers react to different situations (R. L. Chapman et al., 1959). In this study, military workers were subjected to various scenarios, and their ability to adapt and adjust their work processes was evaluated. Additionally, another early look at organizational learning comes from a 1965 study that attempted to use the world of business and decision making to establish a theory of 
organizational learning (Cangelosi \& Dill, 1965). Other foundational works examined organizational learning include (Duncan, 1974; Jelinek, 1979; Miles, Snow, Meyer, \& Coleman Jr, 1978). These studies all focused on attempting to define organizational learning and how organizations can achieve it. While definitions differ, the principal idea from early research is that it is strategically beneficial for organizations to have a culture that promotes organic learning. Furthermore, early research agrees that organizational learning manifests itself as a change that happens to individual workers that are often unnoticed leading to a noticeable difference in the organization.

None of these studies focused on education, and little research exists that discusses organizational learning in a higher education setting. While these works make up the initial groundwork for organizational learning (Cangelosi \& Dill, 1965; R. L. Chapman et al., 1959; Duncan, 1974; Jelinek, 1979; Miles et al., 1978), there has been a significant amount of other research on the topic. One of the critical factors in examining the history of organizational learning is to understand that the study of OLT is based on the different academic disciplines studying it. Economists have tackled organizational learning in the development of new industries and technologies (Attewell, 1992), as well as research and development (Mowery, 1981).

Organizational learning has been studied in the business world for many years, beginning when Cyert and March (1963) outlined its critical role in the 1960s. The relationship between learning and change has often been examined at a strategic management level (Dodgson, 1991; Loveridge \& Pitt, 1990), with insight given into how managers or leaders can use organizational learning to affect organizational change (Noruzy, Dalfard, Azhdari, Nazari-Shirkouhi, \& Rezazadeh, 2013). Additionally, 
organizational learning is a valuable framework because it is widely recognized that learning organizations are more competitive than those that do not embrace the idea (Chiva, 2017; Coopey, 1995; Tsang, 1997). Since change happens rapidly in the world, organizations should be willing and able to learn through experience. Otherwise, they will cease to exist.

Upon reviewing the literature, three key themes arose that will inform the basis of how organizational learning will be used in this research. First, learning generally has positive consequences, even if the outcomes of learning may be negative (Guinot, Chiva, \& Mallén, 2016; Louis, Louis, Murphy, \& Murphy, 2017). For example, an EM office might employ a specific communication piece to prospective students, and they receive overwhelmingly negative feedback about the marking of a program. The outcome was adverse, but the consequence was a positive learning experience for the organization (Mahr, Lievens, \& Blazevic, 2014). Second, although learning is based on individuals, organizations can learn (Reese \& Hunter, 2016). This means that while most of the specific learning that happens in an organization occurs at the individual level, if enough of individual learning happens, the culture of the organization can change, causing organization-wide learning to occur (Cook \& Yanow, 1993). Third, learning occurs throughout all activities of an organization, and it happens at different speeds and levels (Schilling \& Fang, 2014). For example, in an EM office, the "on the ground" recruiters may recognize a change in how prospective students prefer their marketing very early on; however, the marketing department that is on campus may take longer to learn this lesson. The takeaway is for organizational learning to be successful; individual learning 
must happen and then be shared with others in the organization (Langley, Smallman, Tsoukas, \& Van de Ven, 2013).

Furthermore, the existing literature provides a solid foundation in which to evaluate to what extent organizational learning is occurring in an organization. Argote and Miron-Spektor (2011) provided a framework to assess an organization, as well as details how leaders can ensure OL is happening. The theoretical framework that comes from the work of Argote and Miron-Spektor (2011) revealed organizational learning is a process that occurs over time, and thus, aims to show an ongoing life cycle wherein experience is transformed into knowledge that can change an organization. This knowledge is then incorporated into the environment of the organization, changing future behavior, and possible experience and expertise. It is important to note that organizational learning happens within a specific context that includes the organization and the environment in which the organization exists (Drazin, Glynn, \& Kazanijan, 1999). It is through this lens that the organizations studied here will be evaluated.

The ability of an organization to leverage individual knowledge or experience into organizational learning or change is a complex one (Choo, 1996). One of the major challenges is how to take that individual experience and disseminate it across the organization. As Choo (1996) believes, this is a particular challenge as knowledge and expertise is closely held by individuals to stand out amongst their peers. Senge (1990) expands by saying that organizations that are unable to leverage the experience of their employees in this way and may are unable to suffer a type of organizational learning disability. One of the most successful ways to combat this type of organizational issue is to create an organization that creates knowledge. In the 1990s Nonaka and Takeuchi 
(1995) created a comprehensive model that is widely used as the standard by which organizations dynamically create knowledge. They discuss the process of turning tacit knowledge or knowledge that is personal into explicit knowledge, or knowledge that is formal and easy to transmit between individuals and groups (Nonaka \& Takeuchi, 1995). As they explain, tacit knowledge is deeply personal and of little value to the organization. Through their four modes of knowledge conversion, tacit knowledge is converted to explicit knowledge. The four modes are socialization, externalization, combination, and internalization. In summation, the process involves individuals acquiring their tacit knowledge through sharing experiences (socialization) such as observation and imitation; converting that knowledge into sharable concepts (externalization) through the use of concepts such as metaphors, analogies, and examples; combining the explicit knowledge of a number of individuals in the organization (combination); and finally, taking the newly created explicit knowledge and embodying that into the members of the organization as new tacit knowledge (internalization), thereby creating a cycle of knowledge creation and organizational learning (Nonaka \& Takeuchi, 1995).

The historical perspective of organizational learning focuses on what learning looks like at an organizational level, how it can be achieved, and how it can be sustained and replicated. More recent research on organizational learning focuses on how leaders can create an environment in which organizational learning can grow and flourish as well as the impact on marketing (Mena \& Chabowski, 2015) and global strategy (Hotho, Lyles, \& Easterby-Smith, 2015). This is especially relevant to this research as marketing, and the global expansion of one's brand is essential in the world of strategic enrollment management (VanderSchee, 2009). Of particular interest and relevance to the world of 
admissions and strategic enrollment management is stakeholder marketing. Stakeholder marketing refers to the way that an organization creates value for itself through strengthening relationships with stakeholders (Hult, Mena, Ferrell, \& Ferrell, 2011), in this case, prospective students. This theory resonates with strategic enrollment management a great deal, as previously discussed. With all schools needing to recruit to the individual, or the private school model, this direct relationship building marketing approach is the only way schools can reach students in this current environment. This ties in with organizational learning, as universities have had to learn through experience how to best utilize this type of marketing and adjust their practices accordingly. The researchers expect to learn a great deal about the evolution in marketing, especially as it relates to this type of stakeholder marketing, in the data collection process. While this research is focused mainly on domestic recruitment of prospective students to institutions of higher education, the impact of the international market cannot be ignored. When considering international markets and how organizational learning interacts, there is surprisingly little research (Hotho et al., 2015). However, the research that does exist, again focuses on agency or stakeholder relationships, as once cultures are crossed, connecting directly with an individual or group of individuals becomes even more necessary, as well as the situational learning that comes from working with different cultures (Hotho, Saka-Helmhout, \& Becker-Ritterspach, 2014).

As this research is also focused on leadership and how leaders directly impact organizational learning, it is essential to review the relevant literature regarding leadership and organizational learning. From the literature, a connection between servant leadership and organizational learning is present. Multiple studies have examined how 
servant leaders tend to be the individuals who are most likely to create an environment in which organizational learning can occur (Liu \& Shi, 2018; Song, Park, \& Kang, 2015). As we learn from Greenleaf (Greenleaf, 1970), who first discussed the idea of servant leadership, the central tenant of this leadership style is that the followers are of the primary importance. In servant leadership, the leader exists only to lift and move forward the followers. Thus, a leader who helps create an environment where experience leads to learning, which leads to change would be a servant leader. A servant leader is not afraid of change and welcomes it, as opposed to more rigid leaders who create stagnant environments of consistency (Greenleaf, 1970). Additionally, non-servant leaders tend to be results-oriented and focused more on the outcomes, rather than the processes by which the outcomes are achieved. Organizational learning is all about the methods and therefore meshes well with servant leadership (Kaemar, Harris, Andrews, \& Tepper, 2013).

Furthermore, transformational leadership blends well with the idea of organization learning theory, as transformational leadership is the leadership style most often used in evaluating leaders who are leading an organization through change (Sarros \& Santora, 2001). Transformational leaders shape the behavior of their followers by motivating them to achieve beyond expectations by transforming their attitudes, beliefs, and values as opposed to merely being compliant or following orders (Rafferty \& Griffin, 2004). As discussed previously, for organizations to change, the people within need to change, which is the desired outcome of a transformational leader. Therefore, as the members of an organization change, the organization itself can and will change. As members of an organization are led in this manner, they will often increase their level of 
job performance, which in turn positively affects the organization, leading to lasting change (Jyoti \& Bhau, 2016).

This research also focused on using organizational learning theory (Cangelosi \& Dill, 1965; R. L. Chapman et al., 1959) in conjunction with the idea of strategic renewal (Agarwal \& Helfat, 2009; Baden-Fuller \& Volberda, 1997; Crossan \& Berdrow, 2003), implemented within a higher education setting, specifically EM and more specifically, undergraduate admissions. Strategic renewal and organizational learning theory were first linked by March (1991) when he delved into the idea of using organizational learning to examine the relationship between exploring new ideas compared with exploiting old ideas within an organization. While March (1991) never uses the words "strategic renewal," his conclusion that there needs to be a balance between new and old ideas, with each having pros and cons, leads directly to the concept of strategic renewal.

This idea was expanded by Crossan, Lane, and White (1999), who developed a framework based directly on organizational learning and strategic renewal. Their context stems from the idea that organizational learning is the driving force for strategic renewal of an organization. Renewal, by definition, requires that organizations examine themselves and learn new ways to improve, while simultaneously building on successes (Hurst, 1995). Crossan et al. (1999) noted renewal and organization are connected if done correctly, which to them means encompassing the entire organization to make lasting change. The result of their research was the " $4 \mathrm{I}$ framework of organizational learning, intuiting, interpreting, integrating, and institutionalizing" (Crossan et al., 1999, p. 525), which they use to assist companies with change and renewal. The "four I's" are relevant here for how they describe the process of going from individual learning to 
organizational learning, a key premise in this research. Intuiting in this model describes how an individual worker can unconsciously perceive patterns and opportunities for improvement in his or her daily work life (Dutta \& Crossan, 2005). Once that individual interacts or shares those ideas with another, they enter the interpreting phase. This phase is about the final formalization of the idea to the individual and the explanation of the idea to others in the organization. Once multiple people in the organization begin changing their behavior to coincide with the new idea, integrating happens. In this phase, conversations and joint action are vital to making lasting change. The process will initially be informal, similar to a shared behavior, until institutionalizing happens. This final phase is the formal adoption and codification of the new process within the organization (Lawrence, Mauws, Dyck, \& Kleysen, 2005). Specific tasks and duties are assigned out and organizational changes are made to ensure the continuation of the new idea (Crossan et al., 1999). Consequently, if a leader can make change through individual action and throughout the entire organization, the 4I's are key.

Finally, this leads to the concept of Data Driven Decision Making. Marsh, Pane, and Hamilton (2006) made the connection between DDDM and Organizational Learning Theory. They discussed how DDDM within education is modeled on practices from industry, such as "total quality management, organizational learning, and continuous improvement (Marsh et al., 2006).”

\section{Data-Driven Decision Making}

The concept of data-driven decision-making (DDDM) within education stems from research by Popham et al. (1985). This research focused on the K-12 environment and how states began requiring the use of outcome data in school improvement planning 
and strategic planning (Popham, 1987; Popham et al., 1985). Additionally, the No Child Left Behind Act (NCLB, 2001) provided opportunities and incentives for educators to use data, mostly test scores, to secure funding and improvement for their schools. DDDM has been explored mostly within the business or private enterprise world, as education has been slower to adopt the use of statistics and data than the business world (Bontrager, 2004).

March, Pane, and Hamilton (2006) postulated how there are various types of data, and DDDM practitioner is to take the various types of data and turn them into "actionable knowledge." This knowledge informs various decisions, leading to the successful utilization of DDDM (Marsh et al.). The ability to turn the massive amount of data that an enrollment management (EM) office receives into actionable knowledge is a major reason DDDM was chosen in this inquiry. Today, there is more data available for college admissions offices than ever before, and personnel can become overwhelmed by the data. Being able to organize and utilize the data effectively directly impacts the success of an EM team (Natek \& Zwilling, 2014).

While DDDM may come from industry, the idea of using it in education is not new (Popham, 1987; Popham et al., 1985), with a myriad of reforms starting in the 1980s and peaking with the national No Child Left Behind Act of 2001 (NCLB, 2001). As Kowalski (2010) noted, critics of NCLB have often reacted negatively to the laws requirement for Data-Driven Decision Making, highlighting "they (educators) have argued that basing consequential decisions solely on research data is demeaning and precarious, demeaning because the wisdom of educators is devalued and precarious because research data is fallible (p. 3). This is one of the major issues with using data in 
education because some practitioners feel that their experience and anecdotal evidence is superior to hard data. The use of data in PK-12 education became a necessity for administrators (NCLB, 2001) and educators as they began to be held to standards of accountability (Lew \& Nelson, 2016). It has been only recently that the value of a college education has been held to a higher accountability (Webber, 2016; Williams, 2016).

Recent years have seen an explosion of research into how data can be used within offices of enrollment management to become more successful. As noted earlier, resources are becoming more and more limited within higher education, therefore requiring strategic enrollment managers to be precise in their efforts of data analyzation, which is an important tool (Cox et al., 2017; Picciano, 2012). This change in perspective and focus in the research to the use of data in enrollment management naturally led to an additional focus on technology and how it can be used. The research emphasizes that technology is key for offices of enrollment management that are attempting to become data driven, as the sheer volume of data both available and needed to be successful, requires the use of advanced technology such as robust Customer Relationship Management (CRM) systems, Student Information Systems (SIS), and data analytics tools (Attaran, Stark, \& Stotler, 2018; Hubbard, Datnow, \& Pruyn, 2014).

Furthermore, the previously mentioned changes in the higher education landscape, particularly when it comes to funding, makes the need for a shift toward datadriven decision making more necessary. This is especially true for public schools, as the sharpest decline in funding has been from state governments (Sav, 2016). As previously mentioned, the larger public schools have had to change their recruitment and strategic enrollment management efforts to mirror those of smaller private schools, namely 
focusing on recruiting on an individual basis (Bontrager, 2004). For small private schools, this did not require a large leap in technology, as the volume of students being recruited was small. For the average small to mid-size private school, there are a few hundred applications to manage, easily done with simple technol,ogy. However, for a mid-size to large public school there are thousands of applications with hundreds of thousands of "leads" to manage (IPEDS), requiring robust technology, which costs money (Sav, 2016).

Consequently, this need for a more efficient means in which to store and analyze data led to the most recent revolution regarding data-driven decision-making in higher education. The emergence and reliance on high-end customer relationship management systems or CRMs (Sutin, 2018). CRMs are databases that allow a user to store large amounts of data and create efficiencies in workflows. For example, the modern CRM allows offices of enrollment management to create communication plans, track student behavior, plan admission counselor travel schedules and communicate directly with students (Hwang \& Choi, 2019). The outcome of the system utilization is that large schools, recruiting to large volumes of students, can recruit students more individually without the tedious effort of historic individual recruiting. In the past, this individual recruitment would have required days of effort by an admissions counselor. Now, in minutes, a record can be created, and the transition to recruitment is seamless. This all leads to more efficiency in time and money, built on the framework of data-driven decision-making. The principles of DDDM are important at the initial stage of the CRM implementation. Offices of enrollment management use their available data in several ways. First, they use the data to know which students to focus on by utilizing predictive 
modeling. Second, data is used to know how to best communicate with students and the best times to implement those communications. Additionally, the data can be utilized to know how to best engage students in the recruitment process and how to help the student complete all the necessary paperwork to become a student. Subsequentially, data is essential for enrollment managers to know how to best yield the student or close the deal (Levin, 2015).

Upon completion of this review of literature, organizational learning theory, and data-driven decision-making emerged as the obvious frameworks in which to undertake this inquiry. As the focus of this research is on how EM offices adapt, learn, and grow into organizations that strategically use data, OLT provides a clear and accurate lens through which to examine the growth and change of an EM office. Specifically, viewing change as a type of renewal, provides a strong foundation, as evident in the literature, this type of learning process tends to be tumultuous and challenging (Crossan \& Berdrow, 2003). Furthermore, as the desired result of the learning process is an organization that embraces and strategically utilizes data, DDDM is a well-established model through which to approach this inquiry. The key factor being investigated is how an organization uses data strategically. Considering the literature, organizational learning theory and datadriven decision-making emerged as the conceptual frameworks to be utilized in this inquiry. 


\section{Summary}

In this literature review, discussed were many different models of evaluating strategic enrollment management. Emphasized were various ways of approaching this topic, all with relative value providing different perspectives. The existing literature was clear that organizational learning and data-driven decision-making were the most relevant choices when considering what a current EM professional needs to be successful. Many of the other theories reviewed focused on the student perspective, in either the deciding phase or the retention phase. As this research is examining the EM practices themselves, organizational learning (Cangelosi \& Dill, 1965; R. L. Chapman et al., 1959; Duncan, 1974; Jelinek, 1979; Miles et al., 1978) and data-driven decision making (Popham, 1987;

Popham et al., 1985), were decided upon as the conceptual frameworks. These two conceptual frameworks provide the appropriate lenses in which to approach this issue by the current literature and the perceived gaps in the literature. This research will attempt to decrease the existing gap in the literature by investigating how higher education organizations can learn and make decisions based on the data collected through the EM offices within the institutions. 
SECTION FOUR

CONTRIBUTION TO PRACTICE 


\section{Introduction}

As colleges and universities work to recruit students who will retain and graduate from an ever dwindling pool of prospective students, enrollment management professionals are continually exploring ways to improve their processes. There is an impending severe decline in the number of U.S. high school graduating students that has a direct link to the recession of 2008. Population forecasts show that starting in 2026, and there will be between 40,00 to 100,000 less students graduating each year for at least five years (WICHE, 2019).

To combat this, enrollment management professionals need to adapt their practices and understanding of the field of enrollment management to remain successful. The focus of this inquiry was to answer how data-driven decision-making could help enrollment management professionals continue to recruit successful classes of incoming students. Additionally, this inquiry sought to answer if and how organizational learning can happen in conjunction with the use of data-driven decision making and is organizational learning important for a successful enrollment management office.

In this chapter, presented will be the outcomes of the research through the various interviews and focus group discussions. Revealed will be how the organizations utilize data-driven decision-making and create a culture of organizational learning. Furthermore, demonstrated were the value of these practices and how they relate to successful enrollment management. Additionally, presented are the findings to each research question and a summary of the data as it related to each individual research question as well as a one page executive summary to be provided to the schools that participated in the research. 


\section{Research Questions}

The research questions guiding this study were to investigate current offices of enrollment management to assess their use of and success with data-driven decisionmaking and organizational learning.

1. How does the use of enrollment data for data-driven decision-making affect the organizational learning of the University, and what type of learning is derived?

2. How can an EM office create a data-driven recruitment model through organizational learning and implement it successfully, and does it perform equally amongst different student populations?

3. What impact does the predictive model have on recruitment and retention, and is that impact equal across different student populations?

4. Do institutions that have successful EM offices rely solely on data-driven decision-making, or are there elements of evidence-based practice involved?

\section{Connection to Conceptual/Theoretical Framework}

Data-driven decision-making and organizational learning provided the framework for exploring how different universities offices of enrollment management behave. Specifically, the research sought to learn whether data-driven decision-making can directly impact an organizations ability to learn as well as become a learning organization. Existing research suggested organizations that learn have a competitive edge over those that do not (Chiva, 2017; Coopey, 1995; Tsang, 1997). Furthermore, current research and trends in enrollment management show that data-driven decisionmaking is essential for enrollment management offices to be successful in the current 
competitive climate. March, Pane, and Hamilton (2006) suggested that there are various types of data, and the job of the data-driven practitioner is to take the various types of data and turn them into "actionable knowledge." This knowledge informed various decisions, leading to the successful utilization of DDDM (Marsh et al.). Being able to organize and utilize the data effectively directly impacts the success of an EM team (Natek \& Zwilling, 2014).

Recent years have seen an explosion of research into how data can be used within offices of enrollment management to become more successful. Resources are becoming more and more limited within higher education, therefore requiring strategic enrollment managers to be precise in their efforts of data analyzation, which is an important tool (Cox et al., 2017; Picciano, 2012). The research was clear that the use of data is vital for enrollment management offices. However, within that theory, there is not a clear plan for creating an environment in which to implement change or for an organization to become one that uses data in an efficient manner.

Organizational learning, as a framework, focuses on how an organization changes and learns from within. In the world today of limited resources and high competition, it is vital for enrollment management offices to be able to adapt without large external influxes of resources. Little research exists that discusses organizational learning in a higher education setting. The foundational research in OLT make up the initial groundwork (Cangelosi \& Dill, 1965; R. L. Chapman et al., 1959; Duncan, 1974; Jelinek, 1979; Miles et al., 1978) while focusing on the business and military aspects of OLT, and there has been a significant amount of other research on the topic. Economists have tackled organizational learning in the development of new industries and technologies 
(Attewell, 1992), as well as research and development (Mowery, 1981). The relationship between learning and change has often been examined at a strategic management level (Dodgson, 1991; Loveridge \& Pitt, 1990), with insight given into how managers or leaders can use organizational learning to affect organizational change (Noruzy, Dalfard, Azhdari, Nazari-Shirkouhi, \& Rezazadeh, 2013).

Furthermore, the framework of organizational learning theory provided how the data was analyzed. Argote and Miron-Spektor (2011) provided a framework to assess an organization, as well as details how leaders can ensure OL is happening. The theoretical framework that comes from the work of Argote and Miron-Spektor (2011) revealed organizational learning is a process that occurs over time, and thus, aims to show an ongoing life cycle wherein experience is transformed into knowledge that can change an organization. This knowledge is then incorporated into the environment of the organization, changing future behavior and possible experience and expertise. It is important to note that organizational learning happens within a specific context that includes the organization and the environment in which the organization exists (Drazin, Glynn, \& Kazanijan, 1999). The final means of evaluation and connection to the frameworks comes from the idea of strategic renewal. This idea was expanded by Crossan, Lane, and White (1999) who developed a framework based directly on organizational learning and strategic renewal. Their context stems from the idea that organizational learning is the driving force for strategic renewal of an organization. Crossan et al. (1999) noted renewal and organization are connected if done correctly, which to them means encompassing the entire organization to make lasting change. The 
result of their research was the "4I framework of organizational learning, intuiting, interpreting, integrating, and institutionalizing" (Crossan et al., 1999, p. 525).

\section{Participants and Data Collection}

The research for this project was conducted on the campus of two universities. At each university, a senior leader was interviewed (a vice president or a vice provost, both of enrollment management). Additional interviews at each school consisted of an admissions director, an assistant director, and an admissions representative. Also, on both campuses, two focus groups were conducted, one of current students and one of other enrollment management leaders, for a total of four focus groups and eight interviews. The below chart will explain how each interview and focus group will be referenced in the discussion of the results:

Table 1 Abbreviations of Participants

\begin{tabular}{|l|l|}
\hline VP 1 & Vice President/Provost at school \# 1 \\
\hline VP 2 & Vice President/Provost at school \# 2 \\
\hline Director 1 & Director at school \# 1 \\
\hline Director 2 & Director at school \# 2 \\
\hline AD 1 & Assistant Director at school \# 1 \\
\hline AD 2 & Assistant Director at school \# 2 \\
\hline AC 1 & Admissions Counselor at school \# 1 \\
\hline AC 2 & Admissions Counselor at school \# 2 \\
\hline SFG 1 & Student Focus Group at school \# 1 \\
\hline Student Participant 1,2,3,4,5 & Various student participants in the student focus group \\
\hline SFG 2 & Student Focus Group at school \# 2 \\
\hline
\end{tabular}




\begin{tabular}{|l|l|}
\hline Student Participant 1,2,3,4,5 & Various student participants in the student focus group \\
\hline EMFG 1 & Enrollment Management focus group at school \# 1 \\
EM Participant 1,2,3,4,5 & Various EM professional in the EM focus group \\
\hline EMFG 2 & Enrollment Management focus group at school \# 2 \\
EM Participant 1,2,3,4,5 & Various EM professional in the EM focus group \\
\hline
\end{tabular}

Note: Eight interviews, Four focus groups

Questions were designed by the researcher to assess if and how data was being used in admissions and enrollment management as a whole as well as if and how organizational learning was happening. Additional questions were asked to assess leadership and follower behaviors, cultural impact and importance, and the effectiveness of recruitment strategies developed by the enrollment management offices of each university. Interviews and focus groups were recorded and transcribed for ease of analysis. The transcripts were provided to the participants for member checking to ensure accuracy.

\section{Presentation of the Data}

In this section, the results of the study are presented. The analysis procedure addressed the four research questions as well as evaluating the results through the framework of organizational learning. The primary means of evaluation will be the lifecycle process assessment posited by Argote and Miron-Spektor (2011) in conjunction with the tacit to explicit knowledge conversion theory presented by Nonaka and Takeuchi (1995). Answers to the research questions were found by analysis of the transcripts of the multiple focus groups and interviews. 


\section{Research Question One}

How does the use of enrollment data for data-driven decision-making affect the organizational learning of the university, and what type of learning is derived?

At both institutions, it was clear that data was behind almost every decision made in the enrollment management offices. As VP 2 said, "data is the backbone of all decision making in our department." Director 2 supported this idea, "we use data to make every single strategic decision as a management team as well as empower our admissions counselors to use data to determine what high schools they are going to visit." This theme of using data in both strategic planning and day-to-day operations was repeated at all levels at both institutions, in every interview. A direct example of this comes from AC 1, who outlines her planning process for fall recruitment season. She outlines how she starts with lists of her current class of students she is going to be focusing on, reports on the territory itself, the various high schools, relationships with counselors, what types of visits each high school offers, and internally created profiles using predictive modeling that allows her to strategically plan and organize her travel.

Research question one focuses on how the use of data-driven decision-making affects the organizations ability to learn. As one evaluates this through the lens of process assessment, the relationship becomes clear. Argote and Minon-Spektor (2011) argued initially, and experience is transformed to sharable knowledge. The recruitment profiles mentioned previously are one example of this idea. Using previous experience from the admissions and enrollment management team, the EM office has created a predictive model. This model is based on using experience with students and understanding how their behavior in the recruitment process will transform into enrollment behavior. For 
example, Director 2 explained, through experience, they know that if a student engages in certain recruitment events or with certain marketing pieces or if they engage in certain ways with an admissions counselor, their likelihood to enroll goes up. This past information then informs future behavior, and the organization has learned what works and what does not work.

The next phase of the process assessment involves taking that learned knowledge of the individual and sharing it organization wide. An example of this comes from the EMFG2. EM Participant 1 noted through communication with the admissions team; they were recently able to re-evaluate their awarding process and be more efficient in the awarding of aid. In the middle of their awarding cycle for the previous enrollment year, the enrollment goals were changed. This led to many necessary conversations between admissions and financial aid so that they could meet those goals, which had changed not only in overall quantity of students desired but a change in the quality of the incoming class. Basically the goal had been to maintain or grow slightly with a keen eye toward quality or the credentials of the incoming class. In February the goal was changed to increase quantity dramatically and increase quality. Only through their collaborations and use of data on what types of awarding would most likely result in certain behaviors, were they able to achieve those goals. As EM Participant 1 said, "we created a model that allowed us to offer three thousand more scholarships than previously but at the same cost or even slightly less the previous years." By using the data and determining that smaller awards to more students would allow them grow as desired, they were able to increase the class without spending more money. 
This leads to the final aspect of the process assessment cycle, wherein the environment of sharing and learning becomes the culture. Nearly every interview and focus group discussed culture and the importance of it. Director 1 said it clearly, "Culture eats strategy for breakfast." When asked to explain, she explained "that you can have all the data in the world and create perfect strategy, but if you don't have a team to execute it, you won't be successful." This theme was repeated in the EM1 focus group who talked about the impact of their new leader and how the culture of open communication has been key to their success. EM1 Participant 1 noted that "Previously, we had been siloed and didn't understand how our work impacted enrollment management as a whole. By taking down those walls and allowing us all to see how everything works together, we all moved forward together." Another example of how the use of data can help build culture comes from AD 2. She talked about how when she first started; she was amazed at how much data was used in the decision-making and strategic planning processes. She said "More so than anywhere else I have worked, we used data for everything and I couldn't imagine how we could use data more." Now, after being there for almost three years, she reports, "We continue to make more and more decisions using data, and just when I think we can't find any more data points to use, we do and use them to successfully guide our strategic planning." It has now become so engrained in the culture, that the possibility of success without using data is hardly considered. What was once a place that VP 2 described as a place that "wasn't using data at all in my opinion, and was relying more on anecdotal evidence or doing what had always been done" is now so data-driven, employees can not imagine a world without it. 
In conclusion, it is clear that utilizing data to enable data-driven decision making can and does lead to organizational learning. It is also clear that the resulting shifts in culture are vital to success. All participants interviewed and involved in the non-student focus groups noted the importance of culture. By creating a culture where ideas can be shared openly and effectively, whereby leaders listen to ideas and respect the experience of the members of the organization, organizational change can and does happen. The interesting component of this research question is that data alone is not enough, nor is well intentioned leadership. If an organization possesses solid data but has no leadership willing to create culture of communication and mutual respect, very little success will come from the data. An organization could create excellent strategy in this situation, but the carrying out of that strategy would falter. Conversely, if an organization has amazing culture they may be great at the closing the deal portion of recruitment, but their strategy would not be putting them in the best situations to be successful. A merger of the two is essential to success.

\section{Research Question Two}

How can an enrollment management office create a data-driven recruitment model through organizational learning and implement it successfully?

The answer to this question that emerged from the data is grounded in theory, less framed from practice. It is clear from these two universities that they have set up their offices along the path of the process assessment life-cycle model used in this research as an assessment tool. No one at either university said they did so intentionally, but it is clear from the evaluation process, that has indeed happened. Both universities created an environment in which individual learning can become organizational knowledge and 
practice, which in turn creates a culture of collaboration and communication. The results of this research and the impressive success these two vastly different universities have achieved, make it clear that following this model can lead to success. However, it is not as simple as knowing one path to success. There are many paths to success, as well as other universities and organizations that have started down this path and not been successful. In answering this question, the researcher will focus on specific practices and ideas that emerged from the research that can help other enrollment management offices achieve success.

A great example of this comes from AD 2. She explained a situation with an out of state recruiter, specifically focusing on California and the Phoenix area of Arizona. Initially, after reviewing the historic data, a new recruiter position was created to serve California and Arizona. Based on past engagement and enrollment, it was determined that the recruiter would spend the majority of their time in southern California and Phoenix, with little to no travel in Northern California. Marketing campaigns would go to all regions, but specific regional travel was focused as described. Over three years, enrollment grew significantly in southern California, grew slightly in northern California, and remained the same in Phoenix. It was then decided to stop traveling to Phoenix and travel more to northern California. After another three year time period, it was discovered that enrollment continued to grow significantly in southern California, was now growing significantly in northern California, and was remaining the same in Phoenix. The organization learned that on the ground, recruitment efforts were not as important in the Phoenix market, and they could re-allocate the same amount of resources (travel to northern California instead of Arizona) and achieve greater results. Only through the 
process of the recruiter sharing their experiences, which in turn became institutional knowledge was this change possible. This is a direct example of creating a data-driven recruitment plan through the organizational learning process.

Another example comes in the form of scholarship strategy. VP 2 told me about a situation with their scholarship strategy this year. They know that their average SAT score is an 1169 , and it is around that score that they yield the most students. As they work to increase their academic quality of their incoming class, it would be easy to offer large awards to the students that score $1500+$. However, from the use of data, they know those students are not likely to enroll, no matter how much money is offered. Therefore, they decided to focus on students in the 1200-1300 range, and they revised their top scholarship program accordingly. Instead of giving a larger amount to fewer very high scoring students, who yield at a low rate, they gave a lower amount to the $1200-1300$ range students and doubled their yield by spending the same to a little less in scholarship dollars. This is another example of the organization learning through data and implementing a successful change.

Furthermore, when considering this question and how an organization can learn and adapt using data, it is also important to look at different student populations. In this case, specifically comparing how the use of data can affect transfer students compared to first time first-year students. Historically, data on transfer students has been lacking compared to what is available for first-year students. This is due to several factors, the largest one being that there are repositories of data for freshman, such as the ACT or SAT, while there is nothing like that for transfer. However, in this research, it seems clear that using data-driven decision-making is just as valuable for transfer students as it is for 
freshman; it is just harder to get that data. Nearly every person interviewed expressed a desire to have more data on transfer students. Director 2 mentioned that he would like to have more data on transfer students to nail down where they are currently located and when they are interested in transferring as well as opening better channels of communication so that customized recruitment plans could be created. In the EMFG 2, participant 2 talked about how personalized and customized messaging is for freshman as they have so much data on them. For transfer students, that is not the case, as once they fall out of the freshman funnel, unless they are actively engaging with the university, it is hard to keep track of them and their needs. Director 1 also weighed in on transfer students, re-emphasizing the desire to use a data-driven approach, but noting the lack of data. She noted "there is a general lack of data on this side, and it is very messy as the data we do have is not under one place."

Furthermore, the lack of a concentrated area in which to preform search, meaning a platform in which a university can purchase transfer prospects, make recruiting transfer students difficult. Director 1 also mentioned the changing landscape of transfer students for her school specifically, talking about how they are getting more and more students transferring from 4 year schools, not just community colleges. This opens a whole new realm of issues, as you have to figure out what caused their prior poor experience and how you can avoid that upon transfer. In general, there is a definite desire to use a data-driven model for transfers, and, when available, it is successful. However, the overall lack of available transfer data makes this difficult and is a needed change in the industry.

In conclusion, it is clear that quality, trustable data was key to creating an environment of data-driven decision-making. Nearly all participants discussed how one of 
the biggest barriers to relying on data is getting clean data and how that process can take years. One of the biggest problems is getting consistent data across a large organization like a college or university. However, it is also clear that once an enrollment management team has data they trust, the strategic benefits are enormous. Data-driven enrollment managers calculate return on investment on all of their recruitment efforts, which is vital in this climate of reduced budgets. There are numerous examples of how data allows a team to do more with less, which is a significant finding and a strong argument for the necessity of data-driven decision making in enrollment management.

\section{Research Question Three}

What impact does the predictive model have on recruitment and retention, and is that impact equal across different student populations?

Both universities utilize an internally created predictive model in their recruitment process. The predictive models created by these two universities take into account demographic data on the students as well as engagement activities. For example, the more a student interacts with the university in the recruitment process (coming to events, responding to emails, answering phone calls, etc...), the more likely the student is to enroll. This is calculated based on noting interactions over many years of recruitment and then following up with those students to see if they enrolled. Over time a pattern emerges that can be then used to create a mathematic probability or predictive value score. These scores are used by the university in a variety of ways, many of which were identified in this research.

An important point to make at the outset of the discussion on predictive modeling is the importance of clean data and having people in place to interpret the data. Both VP1 
and VP2 mentioned this repeatedly. Both of them specifically described how they revamped their data teams, going through a process of hiring a strong data analysis team and making sure the data being pulled in admissions aligned with the data being pulled from institutional research. VP 2 noted, "What gets measured is what gets done," about the importance of tracking items that you wish to move the needle on, and the only way you can do this is to have good data.

In the EMFG 1, participant 1 and participant 2 talked about using the predictive model as an enrollment management team. They talked about how that was one of the biggest changes with the current leader. Before that leader, admissions had a model score, but it was not shared across the EM team. Now, they all have access to it and use it cohesively as one unit. For example, admissions uses it to develop a specific recruitment strategy for a student; financial aid will use it to help determine scholarship strategy, while marketing uses it to inform communication flow. AC 1 noted how she uses the predictive model to set her entire recruitment strategy, focusing on those students who are most likely to enroll and allocating her time accordingly. This sentiment was echoed by AC 2 as well, noting "everything I do to plan travel starts with historical data and the predictive model."

Another example of the use of predictive modeling comes from VP 1 and ties in the idea of retention. As mentioned previously, the goal is to recruit retainable students, not just admissible students. VP 1 reported about some data analysis he does regarding whether a student will persist or not return and he has it narrowed down to one variable, how they performed on a math placement exam. As he told me "if a student doesn't pass 
the math placement exam and goes into a remedial class, they have about a $70 \%$ chance of continuing. If they pass and go into Calculus immediately, retention is about $80 \%$.

Furthermore, if they have a high grade in the remedial course and continue, they are up to $90 \%$, but if they get a low grade in the remedial it falls to $50 \%$." When he first looked at the data he said "I laughed and told the math faculty we just needed to get rid of remedial math." Obviously that is not the answer, but he talked about the danger of incorrect interpretations of data and how they can use this data to strengthen their remedial math program to better prepare students for calculus and beyond. His point is important as it was echoed across most of the interviews and focus groups, interpretation matters. It is not enough to have clean data; you must have a person or team to analyze it as well as develop strategy.

In conclusion, predictive modeling is important and helpful to enrollment managers when used properly and effectively. This is one area where it would be extremely possible to get overwhelmed in too much data if a solid strategy was not in place to process, analyze, and strategize using the predictive model. Both of these schools do an effective job in gathering clean, usable, and replicable data. Both schools have teams in place with years of experience in creating the models as well as developing strategy from the predictive models. In this case, it is important to note that if a school does not have such teams in place, the use of a predictive model could be of little to no benefit and possibly even harmful. If a school were to collect the wrong data or make incorrect strategic decisions from incomplete data, recruitment strategy could be skewed in a way that would not have happened without the use of the model. As with all tools in 
an enrollment manager's toolbox, this one especially must be used with knowledge and practice.

\section{Research Question Four}

Do institutions that have successful enrollment management offices rely solely on datadriven decision-making, or are there elements of evidence-based practice involved?

For as long as enrollment management has been around, it has existed on a spectrum with art on one end and science on the other. In recent years, with more research focused on understanding enrollment management, more formal training for those involved in the field, and more professional development and sharing of ideas available, the field has moved closer to the science end of the spectrum. One of the unique results from this research was how much anecdotal evidence or elements of evidence-based practice remain in effect at these schools. Nearly everyone mentioned how the relationship side of recruitment is still an important and vital factor and cannot be entirely replaced with data.

VP 1 highlighted how important it is to know the students and discern what matters to them from both a recruitment and retention standpoint. He also discussed how the student experience has become more important during his time in enrollment management. 'It's almost like their experience is what matters, not just programs, but student satisfaction... knowing that if a student does certain things that will lead to more happiness, more student success, and ultimately more retention.”

The idea of feel and the use of evidence-based practice was clear when talking to the student focus groups. Over and over, I heard about "feel" and the "visit experience." SFG 1 participant 1 told me "I came to campus for a visit and fell in love with the 
students, how they explained their programs and how they felt about campus and the whole campus life." This was particularly interesting as admissions offices are more and more turning to utilizing current students in their recruitment practices, not just admissions, and other campus professionals. SFG 2 participant 1 agreed when noting "I came for an overnight campus visit and fell in love. As an out of state student I didn't want to leave my family, but I didn't have much choice after my visit." This also reiterates the power of the campus visit, which is a known commodity among admission professionals. Perhaps the most powerful statement came from SFG 2 participant 2 who summed up her recruitment experience "every other school I visited made me feel like I would be lucky to go there, this school made me feel like they felt lucky to have me." This idea of creating a family was indicated as being as equally or in some cases more important than even financial considerations. SFG1 participant 2 highlighted "I had three offers, one in state and two out of state. I visited all three, and even though this school cost a little more, after all scholarships, I just felt at home here and couldn't see myself at the other two schools."

This aligns to evidence based practice, as there is no measureable data on feel and comfort. However, experienced and creative enrollment managers can put some data behind this idea. While one cannot create an ideal visit experience that is custom to every student, an enrollment manger could survey and talk to prospective and current students to see what matters most to them. Additionally, there are companies that have begun doing market research and creating mindset or profiles on prospective students that can help universities create even more customized marketing based on the specific interests of students. Examples of this comes from Director 1 and AC 1. They both told me about 
a relatively new initiative in their office that they call "passion projects." In short, the director has allowed each admissions counselor to pick one group of students that is important to them to focus on, on top of their assigned territory. For example, AC 1 was a first generation student, so she chose to work with prospective students who are first generation. A purely data-driven approach would have broken up the territories based on demographics, and a specific counselor would work with all students in a specific territory. While they use that approach for general recruitment, for the passion projects a counselor gets to focus on a group they are passionate about. This leads to an positive experience for the student and is personally rewarding for the admissions counselor. AC 1 called it "a non-monetary bonus," a job perk that she gets just from doing her job. That type of approach is a definite mix of data-driven decision making and evidence based practice. One thing is clear from the research; data alone is not enough. A school can know everything possible about a student, deliver a timely and impressive financial aid and scholarship award, have top ranked academic and co-curricular programs, and still miss out on students. The campus visit matters, as does every interaction a student has with a given university.

In conclusion, to be a successful enrollment manager, one must employ elements of both data-driven decision-making and evidence based practice. The student experience still matters inordinately and while one can customize this based on data to some extent, the execution and how you make a student feel will ultimately be what makes a student decide to attend the university. A data driven recruitment plan will not be successful if a university does not have the right people in place to genuinely care about the incoming students. Conversely, having people who care about the prospective students but do not 
know where or how to recruit effectively, will also not lead to success. The successful enrollment management office of the future will rely on data-driven decision making. In turn, the data will inform the practices of a well-intentioned recruitment team who genuinely cares about the needs of incoming students and who can successfully communicate that care by creating a family type environment where the student feels connected and wanted. In short, data informs behavior and the enthusing genuine environment. Maximum performance of enrollment management is enhanced through the blending of the art and science of data decision making.

\section{Conclusion}

From this research, it is suggested data-driven decision-making can be an effective means to create a successful enrollment management office. Through a practice of data-driven decision-making, organizational learning can transpire, which will allow an organization to maintain success and build a culture that sustains that success. Three key themes emerged from the research that will assistance informing best practices for other colleges and universities to implement ideas from this research. These themes are: organizations must have good data; a strong culture can emerge; and, mix of art and science.

\section{Theme One: Organizations must have good data}

First, to have an office that utilizes data-driven decision-making, an organization must have good data. Before a leader attempts to implement the practices of data-driven decision-making, the data must be clean, replicable, and usable. This is especially important if a leader is attempting to create change and move an organization to a more data-driven approach. As VP 2 noted, when she first began, the campus community was 
skeptical of data coming out of admissions, as it had been incorrect for years. She first began the process by cleaning all of their data and then working with others on campus to confirm their data, including brining in institutional research, so admissions data matched data at a university level. This gave admissions credibility across campus when she began presenting ideas and changes to a more data-driven approach. Furthermore, a proper team must be in place to analyze the data and build successful strategy from it. Having clean data is a key first step; however an organization needs the data analytics to take full advantage and become data-driven decision makers.

\section{Theme Two: A strong culture can emerge}

The second key theme that arose from the data relates to culture. Through a process of organizational learning, a strong culture can emerge. If a leader sets the culture to be one that is data-driven, the leader must be willing to listen to and learn from the others on the team. This creates an environment of sharing and communication, which will lead to all members of the organization feel comfortable sharing their experiences, which equals more data. This culture of openness and team first are key to success for any enrollment management office, as this translates to the student experience. If an office is plagued with negative culture, that will reflect in the recruitment process. As noted, students must feel comfortable and like they fit on a campus, and negative internal culture inevitably leads to a negative perception from the prospective students. The best strategy in the world will not work with negative culture. A great example of this goes back to the passion projects mentioned by Director 1 and AC 1. They have set this culture in their office where data is vital but just as vital is finding things you are passionate about and working those as well. By allowing the counselors to engage in work related 
activities that are connected to groups or ideas they are passionate about, work seems less like work, they have grown in enrollment, counselors report greater job satisfaction, and culture is created and improved.

\section{Theme Three: Mix of art and science}

The third and final theme that emerged from the research has to do with the idea of enrollment management being a mix of art and science. Historically, enrollment management was mostly art. There has not always been the large amounts of data available on students that there is today, and there was little to no formal training on how to be an enrollment manager. In 2018, the University of Southern California created the first Masters Degree in Enrollment Management. Since then there are more and more professional development opportunities available for enrollment managers. There is much more data available to use in the recruitment process. However, even with all of that being true, there is still an element of art to the job of enrollment manager. The "feel" of a campus and a visit experience still matters a great deal to students. By utilizing data on what matters to students, an enrollment manager can get some grasp of an idea on how to create an excellent student experience. However, every student is different, and what they want out of their recruitment experience is different. The successful enrollment manager must create a team that can respond to these difference in a consistent and genuine manner. The entire campus must be on board, as well. If the student has a positve experience with admissions, but then a different department on campus or an academic unit creates a negative experience, that is what the student will remember. The successful enrollment manger is a bridge-builder across a university who can successfully 
communicate the idea that recruitment is a campus wide job, not just a job for admissions. 


\title{
Recommendations
}

\section{The recommendations are as follow and will be disseminated through a one page}

\author{
executive summary, a pdf image of the summary is included below.
}

\section{Executive Summary}

As the number of graduating high school students continues to decline, the importance of efficiently recruiting students who will retain and graduate is more important than ever before. Population research suggests that there will be a nearly $5 \%$ decline in the number of graduating high school students in the next 10 years. University enrollment management offices must be prepared for this decline and adjust their practices accordingly.

\section{Methodology}

Interviews and focus groups were conducted with current admission and enrollment management staff as well as current students. Interview and focus group questions were designed to understand how successful colleges and universities use data and data-driven decision-making within enrollment management. Additionally, organizational learning theory was assessed, to see how leaders can create a culture where change can happen from within, eliminating the need for large influxes of external funding to create change.

\section{Results}

- Enrollment management staff report the use of data in nearly all decision making and strategic planning

- Enrollment management staff report that a culture of openness and communication where leadership fosters comfort with sharing of ideas is instrumental in EM success

- Only when employees feel comfortable sharing ideas and experiences, can those experiences lead to an addition to organizational knowledge and in turn, organizational learning

- Students report that a "feel" for fit and the human aspect of recruitment still matters, in some cases being the most important aspect of their decision.

Strategies

- Schools should shift recruitment efforts to more of a private school model, meaning personal attention, individualized recruitment efforts, and personalized experiences.

- Schools should utilize data in a data-driven decision-making model to effectively and efficiently recruit students. Use of data is essential to strategic planning and implementation of that strategic plan for all of enrollment management

- Examples include but are not limited to territory management, distribution of recruitment team, scholarship and financial aid modeling and strategies, efficiency of transcription and credit transfer for transfer students, and communication and marketing strategies

- Schools can no longer rely on brand recognition alone or one size fits all recruitment. Too much data exists and there is too much competition for a declining pool of students to rely on old strategies or to keep doing what you have always done before

- A combination of data use and strong personal recruitment is essential for success Key takeaway quote

"Every other school I visited or talked to made me feel like I was lucky they were considering me, this school made me feel like they were lucky to have me." 
SECTION FIVE

CONTRIBUTION TO SCHOLARSHIP 
Editorial Board,

Higher Education Quarterly

Dear Board;

Please accept this research article submission for review in the next issue of Higher Education Quarterly. The use of data-driven decision making and how it can contribute to organizational learning is a gap in the research. Due to the increased scrutiny of higher education outcomes and the decrease in funds available as a whole to higher education, this article is timely and supports the enrollment management community with implications for practice and recommendations that is a pillar of Higher Education Quarterly.

The article adheres to the content guidelines specific to a research article and includes an abstract, related research, and methodology. Analysis of evidence and implications related to the research are also included in the narrative. The manuscript is less than the maximum 35 pages specified in the publication guidelines. Format submission rules are also followed, as the research article complies with the style and reference directives using the 6th edition of the Publication Manual of the American Psychological Association (APA).

Specifically, the researcher conducted interviews and focus groups at two universities in the United States, a mid-sized private university, and a large, public university. This article reviews current literature surrounding enrollment management, data-driven decision-making, predictive modeling, and organizational learning theory as they related to the recruitment and retention of prospective students into institutions of higher education in the United States. This study promotes awareness and the importance of the use of data and the creation of a culture by leaders to enhance the student recruitment experience and relate it to enrollment management best practices.

Thank you, Justin D. Gragg, Ed.D.

13102 Anthony Drive, St. George, KS 66535

Jdgragg1@gmail.com 


\title{
Title Page
}

\section{Title}

\section{AN EXAMINATION OF ORGANIZATIONAL LEARNING AND DATA DRIVEN DECISION MAKING WITHIN TWO UNIVERSITY ENROLLMENT MANAGEMENT PROGRAMS}

\section{Running title}

DDDM and OLT in EM

\author{
Author \\ Justin D. Gragg \\ Dr. Barbara N. Martin

\section{Author's affiliations}

The authors are affiliated with the University of Missouri as the degree granting institution as well as the University of Central Missouri as the degree is part of a multischool cooperative program.

\section{Main Text File}

1. Title, abstract, and keywords;

\section{Title}

AN EXAMINATION OF ORGANIZATIONAL LEARNING AND DATA DRIVEN

DECISION MAKING WITHIN TWO UNIVERSITY ENROLLMENT

\section{MANAGEMENT PROGRAMS}

\section{Abstract}

According to the United States Department of Education (Hussar \& Bailey, 2016), students graduating between 2018 and 2028 will decrease by 
approximately $4.5 \%$. Historically, recruitment has not worked in collaboration with retention. The focus of this research is to address the declining number of students, and effective strategies universities can use to recruit students who will progress and graduate. Three themes emerged from the research that will help to inform best practices for colleges and universities. The three themes are: that an organization must have good, usable data and the resources needed to analyze that data; an organization should strive to create a culture of openness, communication, and teamwork to sustain success; and enrollment management is a mix of art and science, with the successful enrollment manager being a person who can blend the use of data with creating a well-rounded, meaningful, and impactful student recruitment experience.

\section{Keywords}

Enrollment management, retention, recruitment, data-driven decision-making, organizational learning theory, culture

\section{Main Text}

\section{Introduction}

As the number of United States high school graduates continue to decline, research into the most effective ways to recruit and retain college students becomes increasingly valuable. According to the United States Department of Education (Hussar \& Bailey, 2016), students graduating between now and 2028 will decrease by approximately $4.5 \%$. Thus, all universities will be competing within the same declining pool of students. Although there has been extensive research on best practices in college 
admission, most of this focuses on qualitative studies with small sample sizes at nonrepresentative or outlier type institutions (Bontrager, 2004). Historically, when focusing on enrollment numbers, college admission offices have recruited students in the door and then let other departments at the university worry about retaining them. In today's competitive market, this is no longer a feasible option. Institutions of higher education should focus on recruiting students who they can retain and graduate (Elliott \& Healy, 2001). Similarly, rising costs associated with college means admissions offices can no longer recruit students who only stay in college a short time. When students do not graduate, the overall net revenue of the university is affected, and schools will struggle to remain fiscally viable (Hussar \& Bailey, 2016).

The focus of this research was to address the declining number of students, and the effective strategies universities can use to recruit students who will progress and graduate. Researchers Kretchmar and Memory (2010) and Seeman and O’Hara, (2006) concurred that to retain students is to attempt to understand students beyond the numbers, looking at students as more than a GPA or test score, and understand what the students are desiring in a college and be prepared to sell the student on that experience. Today's students have more choice and power than ever before in their college search, and universities should adapt if they want to remain competitive (Kretchmar \& Memory, 2010; Seeman \& O'Hara, 2006; Stewart \& Heaney, 2013). Therefore, this study will approach the overarching question, How can universities and enrollment management (EM) offices effectively use data to recruit and retain students? Furthermore, how can enrollment management offices learn and adapt to new strategies and make themselves available to learn and create new strategies? 


\section{Statement of the Problem}

Within the review of the literature, the identified problem is that there is little to no research on how higher education organizations learn and adapt to use data-driven decision-making. The current research on the use of data-driven decision-making is in the K-12 environment. Legislation has forced K-12 to be more data-driven(Greene \& Forster, 2003; NCLB, 2001). However, the research is lacking when it comes to higher education, as most of the literature discusses the need for using data (Mandinach, 2012; Marsh, Pane, \& Hamilton, 2006), how to collect and disseminate data (Price \& Kirkwood, 2014), and how to use it to inform practice from an institutional level (Sailesh, Lu, \& Al Aali, 2016), not within enrollment management (Hora, Bouwma-Gearhart, \& Park, 2017). Additionally, there is a literature gap when it comes to using this data within a learning organization.

Often in higher education, there are examples of universities creating a new program due to an initiative from the administration. Consequently, that is not a new process that comes from organizational learning (Cangelosi \& Dill, 1965; R. L. Chapman, Kennedy, Newell, \& Biel, 1959), but rather a system that comes from a directive. Researchers Levitt and March (1988) revealed when organizations learn through experience and make changes based on evidence; those changes tend to be lasting and are more profound. While research exists on successful EM strategies and initiatives (Bontrager, 2004; DesJardins, Ahlburg, \& McCall, 2006; Dolence, 1998), there remains a gap in researching ways for those programs to occur organically and without special funding (Jose, Kurian, \& Biju, 2016; Miller \& Bell, 2016). 
In an attempt to place this issue into perspective through a review of the literature, the researcher noticed several challenges facing admissions offices, and these challenges have not changed over time. First, as technology and the needs of students change, admissions offices must be willing and able to adapt to the needs of the student (Lindbeck \& Fodrey, 2010). The days of the Field of Dreams model of recruitment, "the we are here, and students will find us" model, are over. Even the most selective of schools are having to recruit in ways they never had to in previous years (Hill \& Winston, 2010). Another major challenge is how to replicate best practices at other schools that do not have the same resources. Most research on best practices focuses on programs that have above average resources, regarding personnel and monetary (Bontrager, 2004).

Furthermore, a third challenge is meeting students where they are currently (Ford, 2011; Levine \& Dean, 2013). For example, ten years ago, email was the most effective way to reach students, ten years prior, phone calls and traditional mail, and in present time, texting is what students want. Compounding this problem, students want different types of communication for different types of information. For example, students tend to prefer text communication for data based information such as dates of events; however they still tend to prefer email or phone calls for informational types of communications (Gikas \& Grant, 2013). This study will attempt to examine how peer institutions handle these challenges and effectively reach students (Ford, 2011; Lindbeck \& Fodrey, 2010; Mora, 2003; Seeman \& O'Hara, 2006).

Additionally, this research addressed how to connect with special populations of students (Cegler, 2012). Special populations can include first-generation students (Irlbeck, Adams, Akers, Burris, \& Jones, 2014), honor students (Nichols \& Chang, 2013), 
students in a specific major or field, such as STEM (Moakler \& Kim, 2014) or liberal arts (Baker \& Baldwin, 2015), athletes (Magnusen, Kim, Perrewé, \& Ferris, 2014), dual credit students (Lile, Ottusch, Jones, \& Richards, 2017), transfer students (Foote, Kranzow, \& Hinkle, 2015), and any number of other groups. Unfortunately, these groups present their own recruiting challenges. Different strategies are needed for diverse populations; one size fits all recruitment is no longer relevant (Cegler, 2012; Young \& Johnson, 2004). Finally, additional research is needed that focuses on the best practices that are replicable across various types of institutions and is cost-effective (Francis, 2014).

In this day of reduced funding through all higher education (John, 1990;

Tschechtelin, 2011), institutions cannot wait to create change when they receive special funding or new programs. Institutions must create an environment where change can happen organically through organizational learning (Ponnuswamy \& Manohar, 2016), and with evident, data-driven results. Data utilization is the most effective and efficient way to display that something works, at least in the current environment of higher education (Hossler \& Kalsbeek, 2013). Therefore, examining how a higher education EM office, specifically an undergraduate admissions office, can learn to use data to create and integrate a predictive model successfully is the focus of this inquiry.

\section{Purpose of the Study}

The purpose of the study using two universities of various size and student population was to examine if and how organizational learning takes place within the EM functions of each school. Use of data-driven decision-making will be considered especially as it relates to success in EM. For purposes of this study, EM success will be 
defined using metrics the institutions themselves have identified. For example, most public institutions are focused on growing enrollment with academic quality (Baum, Kurose, \& McPherson, 2013). This means these schools want to increase the size of their incoming student population while also increasing the academic profile of their incoming class. Using standardized test scores, high school GPA, and class rank from high school class or other individual measures schools have created can be measured.

Contrary to this, smaller private schools are often less concerned with enrollment growth and more focused on the academic quality of their incoming class (Bontrager, 2004). However, with recent budget cuts to higher education, nearly all institutions are looking to grow enrollment (Calderon \& Mathies, 2013). Therefore, for this research, the primary indicator of EM success will be the percentage growth of the incoming freshman and transfer class.

The researchers focused on what types of collected data and what kinds of decisions are made from that data. Specifically, the focus was to compare various types of organizational learning that comes from multiple types of data, as well as decisionmaking models used based on specific data being collected. The examination of the use of certain types of data-driven decision making that lead to particular outcomes will also occur with specific regard to the mission of the institution. For example, how do institutions focused on maintaining enrollment compared to growing enrollment make different types of decisions? Do they gather different types of data?

\section{Research Questions}

The following research questions guided this research:

1. How does the use of enrollment data for data-driven decision-making affect 
the organizational learning of the University, and what type of learning is derived?

2. How can an EM office create a data-driven recruitment model through organizational learning and implement it successfully, and does it perform equally amongst different student populations?

3. What impact does the predictive model have on recruitment and retention, and is that impact equal across different student populations?

4. Do institutions that have successful EM offices rely solely on data-driven decision-making, or are there elements of evidence-based practice involved?

\section{Introduction to the Scholarly Review}

Estimates reveal the number of high schools graduating students are declining in the United States in all but two states, Florida and Texas (Hussar \& Bailey, 2016). This highlights that offices of Enrollment Management (EM) within institutions of higher education should learn how to be more effective and efficient when recruiting students. In reviewing the current research on this topic, trends begin to emerge quickly. There is a great deal of literature on national best practices within enrollment management, and how enrollment management is similar to marketing (Berry, 1995; Bontrager, 2004; Hossler \& Kalsbeek, 2013; Payne \& Frow, 2005). Similarly, how to integrate enrollment management to serve the university effectively has been investigated (Bowen, Bok, \& Burkhart, 1998; DesJardins et al., 2006; DesJardins et al., 1999; Hossler \& Kalsbeek, 2013). This research all focuses on either the role of a successful EM office or the strategies that successful EM offices use for decision-making. Conversely, there is a lack 
of research on how an EM office becomes such an entity that can effectively use these strategies.

It is from this gap in the literature review, whereby these researchers are seeking to examine how an EM office can become effective in utilizing all the strategies the existing research discusses. The focus of this examination were two institutions that have changed at the fundamental level of enrollment management, with little to no outside funding, and have created a predictive model of recruitment and an overall model of sustainability about successful enrollment management. Specifically, this review of literature provided an understanding of the constructs involved, present evidence of their connectivity, and provide a conceptual basis for the investigation.

There are some potential lenses in which to view this problem, most notably retention theory, as put forth by Tinto (1987) in the 1980s. Other approaches initially examined were the College Choice model (Paulsen, 1990), Predictive Enrollment Theory (DesJardins, 2002; Goenner \& Pauls, 2006), and Evidence-Based Practice (Lodge \& Matthews, 2017; Slavin, 2002).

\section{Retention Theory}

Historically much of the research on EM and admissions issues was focused through the lens of retention (Tinto, 1987), as it was both an uncomplicated way to measure success and a national statistic that was gathered by all institutions of higher education. Specifically, through this literature review, examined was retention theory as a possible theoretical framework from which to approach this research. Retention theory is based mostly on the work of Tinto, who focused on the individual characteristics of students and what they bring to the table (Tinto, 1987, 2006). Tinto's theories are the 
foundation of studying retention in education, and there are numerous studies using retention theory as a lens in which to examine EM (Davidson \& Wilson, 2013; Stanton et al., 2017).

Most of the recent research on Tinto's work is focused on retaining specific groups, such as racial and ethnic minorities (Museus, 2014; Strayhorn, 2014), online and distance education students (Freitas, Morgan, \& Gibson, 2015), and males (Voyer \& Voyer, 2014). As colleges and universities become more and more adept at strategic enrollment management and identifying specific populations they wish to target in the recruitment process; the focus has become more on targeted recruitment and retention, rather than admitting anyone who will attend (Berry, 1995; Bowen et al., 1998). This leads to the idea of crafting a class (Ehrenberg, Zhang, \& Levin, 2005), which is more about identifying an incoming class of students that have a particular set of characteristics, compared to allowing anyone possible into the university. While the research agreed this is an essential aspect of strategic enrollment management (Bontrager, 2004; Hossler \& Kalsbeek, 2013), when viewed through the lens of retention theory, the focus is students that possess specific characteristics and, not on how the organization can become one that can identify those characteristics.

Furthermore, current research focused on retention theory is primarily concerned with the millennial generation of students and their specific needs, not the organization (Turner \& Thompson, 2014). Moreover, the research regarding millennials and retention focuses on the parent-student relationship (Barton \& Hirsch, 2016), the faculty-student relationship (Romsa, Bremer, \& Lewis, 2017), and the relationships between students and all campus services (Sidelinger, Frisby, \& Heisler, 2016). This research is important and 
helpful when discussing student success and retention; however, for purposes of this research, it is on student factors. While the analysis does consider interactions with the campus community, this research study will analyze student characteristics and behavior. Another idea that is often considered when looking at retention is the usefulness or success of orientation and first-year success programs (Bell, Gass, Nafziger, \& Starbuck, 2014; Permzadian \& Credé, 2016; Wibrowski, Matthews, \& Kitsantas, 2017). While these inquiries bring us closer to learning about institutional programs as opposed to student characteristics, this research is focused on the enrollment management functions of a university, not the student success functions. Whereas some enrollment offices do house orientation, the focus of this research is on the recruitment aspect of the enrollment cycle, not student life and retention.

As stated previously, retention theory is focused on the factors a student possesses that make him or her more or less likely to retain (Davidson \& Wilson, 2013; Tinto, 1987, 2006), rather than how an organization can learn and change to better recruit students. This research study focuses on the internal processes within organizations and how organizations can learn to predict better how a student will make their college choice. The next model examined brings us closer to that idea, as it examines the college choice model, unlike retention theory that centers on the student factors that affect retention, not initial college choice.

\section{College Choice Model}

The College Choice Model comes from various researchers who proposed the three-phase model of choice, most notably Jackson's (1982) work in the 1980s. Jackson suggested the initial three-phase model of choice, the aspiration formation stage, the 
college search and application phase, and finally, the selection and attendance phase. The aspirational phase is when the student begins to consider what his or her preferences are when it comes to college. While Jackson (1982) stated many factors affect preference, the three leading factors are previous academic achievement, context (peers choices, location, and type of college), and family background. The search and application phase is typified more on exclusion Jackson (1982) noted, observing there are typically a large number of colleges that fit a student's basic criteria list, so exclusions should be made on outlying factors such as the preference factors mentioned above along with the significant cost consideration. With college more expensive today than when Jackson's research was conducted, it could be argued that cost is an even more significant preference factor for students today. Paulsen's (1990) work on student enrollment behaviors in the 1980s and 1990s, especially as it relates to cost, continued Jackson's work and began tying its importance to enrollment and the declining student populations. Paulsen focused on the real cost of attending college and the cost-benefit ratio of a college education, which, again, with the rising cost of college only becomes more relevant for today's student (Paulsen, 1990; Paulsen \& John, 2002)

On the third and final phase, Jackson (1982) noted the evaluation or selection phase. One interesting point he made is while research is focused on this area, he argues that this state is somewhat anti-climactic, with the decision essentially being made in the other two phases, albeit often subconsciously. When a student makes a list of the essential factors and begins eliminating options, often a winner emerges, with the choice being more of a result of only one school hitting the marks, as opposed to a conscious decision. 
Most of the additional work on college choice uses this three-phase model as its basis. Similarly, Hossler and Gallagher (1987) introduced their own, similar, three-phase model, exploring the role of socioeconomic status in the college choice process, something that Jackson did not consider. As mentioned above, the cost was not the issue in 1982 as it is today, so socioeconomic status and cost become more and more prevalent in the college choice research as time progresses (Skinner, 2018). Other key introductions that Hossler and Gallagher (1987) made include the specific role of parents in the choice process, the value of interactions with higher education organizations (particularly of interest in this research), and how and when higher education institutions distribute information to prospective students. In addition to the key insights provided by their study, Hossler and Gallagher set the stage for the next wave of research into college choice by beginning the focus on individual or special populations of prospective students.

There is a large body of research on college choice among special populations such as race and ethnicity (Manski \& Wise, 1983; Perna, 2000, 2006), family income (An \& Sorensen, 2017; Brown, Wohn, \& Ellison, 2016; John, 1990; Paulsen \& John, 2002), and parent education (Chiu et al., 2016; Kansal \& Kaur, 2016; Manski \& Wise, 1983). The current research on choice when related to race and ethnicity and family income tend to overlap. The investigation revealed both students of color and students from low socioeconomic backgrounds are less prepared for higher education that white middle and upper-class students, as well as having less access to higher-level math courses in their high schools (Adelman, 2006). Solorzano and Ornelas (2004) also noted schools with fewer resources, and those that are populated mainly by students of color offer fewer 
advanced placement courses. The research mentioned above and other research focused on parent education, and family income also tends to be grouped with socio-economic status. The education level of the parent is often a direct reflection of the socio-economic status of the family (Kao \& Thompson, 2003). This issue also becomes intertwined with race, as researchers have noted the increasing stratification of higher education based on race, ethnicity, and social class (Freeman, 1997).

While this model does focus on the deciding phase of the EM life cycle, there is information on student behavior, not organization behavior. Additionally, similar to retention theory, the focus here is on student factors, not organizational factors.

Furthermore, there is no focus on data output, or the use of quantitative data, in a strategic manner (R. Chapman, 1984; Hossler \& Gallagher, 1987; Paulsen, 1990). As shown, the prevailing theories historically used to evaluate higher education focus on student factors, not organizational ones, as well as lacking a depth of focus on data. The next model to be discussed takes the discussion one-step further and introduces an analytic and historically business-driven process of data analysis called predictive modeling.

\section{Predictive Modeling}

Predictive modeling has its roots in the fields of economics, business, and finance, and has been used in all facets of life, including predicting the success rates of marriages and careers (Eskreis-Winkler et al., 2014). In recent years, predictive modeling has led the way for data mining (Rygielski, Wang, \& Yen, 2002), a similar process, but one that is focused on consumer behavior. Data mining has even made its way into education. However, similar to data mining as a consumer behavior tool, data mining in education is 
focused on improving the consumer (student) experience rather than predicting behaviors (Romero \& Ventura, 2013). The use of predictive modeling in education was first examined by Desjardins (2002) and Thomas, Dawes, and Reznik (2001). One primary reason for the lack of research in this area is the underlying, arguably incorrect, assumption, which institutions of higher education lack the in-house expertise to create an accurate predictive model (Goenner \& Pauls, 2006).

A predictive model in this context is a statistical model used to predict enrollment, based on information gathered on prospective students and students that enroll (DesJardins et al., 1999). Additionally, some predictive models are used in retention rather than enrollment function. For example, some institutions of higher education use predictive models to anticipate students that may be at risk for dropping out (Thammasiri, Delen, Meesad, \& Kasap, 2014), especially in at-risk populations (Márquez-Vera et al., 2016). In recent years, institutions of higher education have doubled down on the inhouse expertise in this area. Offices of Institutional Research, once run by educators, have begun to be taken over by economists and mathematicians, leading to a renaissance in predictive modeling in education (Langston \& Loreto, 2017). While the early use of predictive modeling focused on items such as loyalty and choice (Vianden \& Barlow, 2014), current predictive modeling is being used by strategic enrollment managers to craft their incoming classes in a proactive rather than reactive way (Bontrager, 2004). Big data and the use of predictive analytics has become pervasive in all of higher education, but nowhere more so than in EM (Daniel, 2015). The pace at which the business of education now moves and the sheer volume of data available to enrollment managers makes the use of predictive modeling not a luxury as before, but rather a necessity. 
However, for this research, predictive modeling does not go far enough. Predictive modeling is an excellent tool to use for an enrollment management office (Bontrager, 2004; D. Hossler \& D. Kalsbeek, 2013). However, it is not a structure in which one can organize an office, it is merely a tool. This research is focused on how an organization can implement a new style of operating to both tell its story and be successful.

\section{Evidence-Based Practice}

Evidence-Based Practice (EBP) is similar to Data-Driven Decision Making (DDDM) and is interchangeably in the literature with DDDM. The critical difference, however, is that EBP is focused on using evidence as a starting point, while still including qualitative factors when making decisions (Price \& Kirkwood, 2014). DDDM is focused on using data and data alone, specifically, statistical types of data. EBP is a strong model when evaluating education as a cohesive unit (Biesta, 2007). However, for this research, data is the focus, and EBP is too broad for the purpose undertaken here (Marsh \& Farrell, 2015). Like Predictive Modeling and DDDM, EBP has its roots in the business world as well as in the medical field (Isaacs, 2003). The business world has never relied on anecdotal evidence, requiring concrete and replicable statistical data, while the medical field is known for genuinely randomized studies that are purely quantitative. These types of studies are nearly impossible in the area of education, as they involve withholding a variable from one group in the study (Biesta, 2007). As education research usually consists of the administration of a new type of program, it would be considered unethical to withhold services to students (Creswell, 2014).

Education has begun to catch up in the last 50 years, and EBP was one of the first significant steps towards that goal (Biesta, 2007; Levant \& Silverstein, 2005; Slavin, 
2002). In general, one of the early concerns with EBP and the use of data in education was the integrity and reliability of that data (Biesta, 2007). However, as educators and education administrators have become more adept with EBP, data use has increased dramatically. Some educators still fight against the use of EBP or DDDM within education, instead relying on their stories and experiences to prove successes (Kowalski \& Lasley, 2010).

\section{Conceptual Frameworks}

Ultimately, this review of the relevant literature led the researchers to organizational learning theory (OLT) and data-driven decision-making (DDDM) as the two conceptual frameworks in which to review this investigation, both discussed in detail in the following sections. The critical element of OLT that leads to its use as the conceptual framework is that the data will be focused on organic, internal change. For these new methods of EM to be successful and sustainable, they must come from within the university. It is vital that organizations learn how they can make this change happen themselves, and for that to happen, individuals must understand how organizations learn (Hoppes \& Holley, 2014).

The stated goal of this change process and the desired outcome, according to the inquiry, is for organizations to be able to create a predictive model of their own, based on their data and resources. Institutions should evaluate their ability to collect data, analyze data, and then eventually make decisions based on that data if a predictive model can ever be achieved (Bouwma-Gearhart \& Collins, 2015). Additionally, regarding DDDM, institutions are being required to collect and present statistical evidence to justify funding 
more now than ever before (Hussar \& Bailey, 2016), particularly with the increase in performance-based funding models (Rutherford \& Rabovsky, 2014).

\section{Organizational Learning}

Organizational learning theory (OLT) primarily originates from research in the late 1950s and early 1960s. The first mention of organizational learning comes from a 1959 study that examined how air defense workers react to different situations (R. L. Chapman et al., 1959). In this study, military workers were subjected to various scenarios, and their ability to adapt and adjust their work processes was evaluated. Additionally, another early look at organizational learning comes from a 1965 study that attempted to use the world of business and decision making to establish a theory of organizational learning (Cangelosi \& Dill, 1965). Other foundational works examined organizational learning include (Duncan, 1974; Jelinek, 1979; Miles, Snow, Meyer, \& Coleman Jr, 1978). These studies all focused on attempting to define organizational learning and how organizations can achieve it. While definitions differ, the principal idea from early research is that it is strategically beneficial for organizations to have a culture that promotes organic learning. Furthermore, early research agrees that organizational learning manifests itself as a change that happens to individual workers that are often unnoticed leading to a noticeable difference in the organization.

None of these studies focused on education, and little research exists that discusses organizational learning in a higher education setting. While these works make up the initial groundwork for organizational learning (Cangelosi \& Dill, 1965; R. L. Chapman et al., 1959; Duncan, 1974; Jelinek, 1979; Miles et al., 1978), there has been a significant amount of other research on the topic. One of the critical factors in examining 
the history of organizational learning is to understand that the study of OLT is based on the different academic disciplines studying it. Economists have tackled organizational learning in the development of new industries and technologies (Attewell, 1992), as well as research and development (Mowery, 1981).

Organizational learning has been studied in the business world for many years, beginning when Cyert and March (1963) outlined its critical role in the 1960s. The relationship between learning and change has often been examined at a strategic management level (Dodgson, 1991; Loveridge \& Pitt, 1990), with insight given into how managers or leaders can use organizational learning to affect organizational change (Noruzy, Dalfard, Azhdari, Nazari-Shirkouhi, \& Rezazadeh, 2013). Additionally, organizational learning is a valuable framework because it is widely recognized that learning organizations are more competitive than those that do not embrace the idea (Chiva, 2017; Coopey, 1995; Tsang, 1997). Since change happens rapidly in the world, organizations should be willing and able to learn through experience. Otherwise, they will cease to exist.

Upon reviewing the literature, three key themes arose that will inform the basis of how organizational learning will be used in this research. First, learning generally has positive consequences, even if the outcomes of learning may be negative (Guinot, Chiva, \& Mallén, 2016; Louis, Louis, Murphy, \& Murphy, 2017). For example, an EM office might employ a specific communication piece to prospective students, and they receive overwhelmingly negative feedback about the marking of a program. The outcome was adverse, but the consequence was a positive learning experience for the organization (Mahr, Lievens, \& Blazevic, 2014). Second, although learning is based on individuals, 
organizations can learn (Reese \& Hunter, 2016). This means that while most of the specific learning that happens in an organization occurs at the individual level, if enough of individual learning happens, the culture of the organization can change, causing organization-wide learning to occur (Cook \& Yanow, 1993). Third, learning occurs throughout all activities of an organization, and it happens at different speeds and levels (Schilling \& Fang, 2014). For example, in an EM office, the "on the ground" recruiters may recognize a change in how prospective students prefer their marketing very early on; however, the marketing department that is on campus may take longer to learn this lesson. The takeaway is for organizational learning to be successful; individual learning must happen and then be shared with others in the organization (Langley, Smallman, Tsoukas, \& Van de Ven, 2013).

Furthermore, the existing literature provides a solid foundation in which to evaluate to what extent organizational learning is occurring in an organization. Argote and Miron-Spektor (2011) provided a framework to assess an organization, as well as details how leaders can ensure OL is happening. The theoretical framework that comes from the work of Argote and Miron-Spektor (2011) revealed organizational learning is a process that occurs over time, and thus, aims to show an ongoing life cycle wherein experience is transformed into knowledge that can change an organization. This knowledge is then incorporated into the environment of the organization, changing future behavior, and possible experience and expertise. It is important to note that organizational learning happens within a specific context that includes the organization and the environment in which the organization exists (Drazin, Glynn, \& Kazanijan, 1999). It is through this lens that the organizations studied here will be evaluated. 
The ability of an organization to leverage individual knowledge or experience into organizational learning or change is a complex one (Choo, 1996). One of the major challenges is how to take that individual experience and disseminate it across the organization. As Choo (1996) believes, this is a particular challenge as knowledge and expertise is closely held by individuals to stand out amongst their peers. Senge (1990) expands by saying that organizations that are unable to leverage the experience of their employees in this way and may are unable to suffer a type of organizational learning disability. One of the most successful ways to combat this type of organizational issue is to create an organization that creates knowledge. In the 1990s Nonaka and Takeuchi (1995) created a comprehensive model that is widely used as the standard by which organizations dynamically create knowledge. They discuss the process of turning tacit knowledge or knowledge that is personal into explicit knowledge, or knowledge that is formal and easy to transmit between individuals and groups (Nonaka \& Takeuchi, 1995). As they explain, tacit knowledge is deeply personal and of little value to the organization. Through their four modes of knowledge conversion, tacit knowledge is converted to explicit knowledge. The four modes are socialization, externalization, combination, and internalization. In summation, the process involves individuals acquiring their tacit knowledge through sharing experiences (socialization) such as observation and imitation; converting that knowledge into sharable concepts (externalization) through the use of concepts such as metaphors, analogies, and examples; combining the explicit knowledge of a number of individuals in the organization (combination); and finally, taking the newly created explicit knowledge and embodying that into the members of the 
organization as new tacit knowledge (internalization), thereby creating a cycle of knowledge creation and organizational learning (Nonaka \& Takeuchi, 1995).

The historical perspective of organizational learning focuses on what learning looks like at an organizational level, how it can be achieved, and how it can be sustained and replicated. More recent research on organizational learning focuses on how leaders can create an environment in which organizational learning can grow and flourish as well as the impact on marketing (Mena \& Chabowski, 2015) and global strategy (Hotho, Lyles, \& Easterby-Smith, 2015). This is especially relevant to this research as marketing, and the global expansion of one's brand is essential in the world of strategic enrollment management (VanderSchee, 2009). Of particular interest and relevance to the world of admissions and strategic enrollment management is stakeholder marketing. Stakeholder marketing refers to the way that an organization creates value for itself through strengthening relationships with stakeholders (Hult, Mena, Ferrell, \& Ferrell, 2011), in this case, prospective students. This theory resonates with strategic enrollment management a great deal, as previously discussed. With all schools needing to recruit to the individual, or the private school model, this direct relationship building marketing approach is the only way schools can reach students in this current environment. This ties in with organizational learning, as universities have had to learn through experience how to best utilize this type of marketing and adjust their practices accordingly. The researchers expect to learn a great deal about the evolution in marketing, especially as it relates to this type of stakeholder marketing, in the data collection process. While this research is focused mainly on domestic recruitment of prospective students to institutions of higher education, the impact of the international market cannot be ignored. When 
considering international markets and how organizational learning interacts, there is surprisingly little research (Hotho et al., 2015). However, the research that does exist, again focuses on agency or stakeholder relationships, as once cultures are crossed, connecting directly with an individual or group of individuals becomes even more necessary, as well as the situational learning that comes from working with different cultures (Hotho, Saka-Helmhout, \& Becker-Ritterspach, 2014).

As this research is also focused on leadership and how leaders directly impact organizational learning, it is essential to review the relevant literature regarding leadership and organizational learning. From the literature, a connection between servant leadership and organizational learning is present. Multiple studies have examined how servant leaders tend to be the individuals who are most likely to create an environment in which organizational learning can occur (Liu \& Shi, 2018; Song, Park, \& Kang, 2015). As we learn from Greenleaf (Greenleaf, 1970), who first discussed the idea of servant leadership, the central tenant of this leadership style is that the followers are of the primary importance. In servant leadership, the leader exists only to lift and move forward the followers. Thus, a leader who helps create an environment where experience leads to learning, which leads to change would be a servant leader. A servant leader is not afraid of change and welcomes it, as opposed to more rigid leaders who create stagnant environments of consistency (Greenleaf, 1970). Additionally, non-servant leaders tend to be results-oriented and focused more on the outcomes, rather than the processes by which the outcomes are achieved. Organizational learning is all about the methods and therefore meshes well with servant leadership (Kaemar, Harris, Andrews, \& Tepper, 2013). 
Furthermore, transformational leadership blends well with the idea of organization learning theory, as transformational leadership is the leadership style most often used in evaluating leaders who are leading an organization through change (Sarros \& Santora, 2001). Transformational leaders shape the behavior of their followers by motivating them to achieve beyond expectations by transforming their attitudes, beliefs, and values as opposed to merely being compliant or following orders (Rafferty \& Griffin, 2004). As discussed previously, for organizations to change, the people within need to change, which is the desired outcome of a transformational leader. Therefore, as the members of an organization change, the organization itself can and will change. As members of an organization are led in this manner, they will often increase their level of job performance, which in turn positively affects the organization, leading to lasting change (Jyoti \& Bhau, 2016).

This research also focused on using organizational learning theory (Cangelosi \& Dill, 1965; R. L. Chapman et al., 1959) in conjunction with the idea of strategic renewal (Agarwal \& Helfat, 2009; Baden-Fuller \& Volberda, 1997; Crossan \& Berdrow, 2003), implemented within a higher education setting, specifically EM and more specifically, undergraduate admissions. Strategic renewal and organizational learning theory were first linked by March (1991) when he delved into the idea of using organizational learning to examine the relationship between exploring new ideas compared with exploiting old ideas within an organization. While March (1991) never uses the words "strategic renewal," his conclusion that there needs to be a balance between new and old ideas, with each having pros and cons, leads directly to the concept of strategic renewal. 
This idea was expanded by Crossan, Lane, and White (1999), who developed a framework based directly on organizational learning and strategic renewal. Their context stems from the idea that organizational learning is the driving force for strategic renewal of an organization. Renewal, by definition, requires that organizations examine themselves and learn new ways to improve, while simultaneously building on successes (Hurst, 1995). Crossan et al. (1999) noted renewal and organization are connected if done correctly, which to them means encompassing the entire organization to make lasting change. The result of their research was the "4I framework of organizational learning, intuiting, interpreting, integrating, and institutionalizing” (Crossan et al., 1999, p. 525), which they use to assist companies with change and renewal. The "four I's" are relevant here for how they describe the process of going from individual learning to organizational learning, a key premise in this research. Intuiting in this model describes how an individual worker can unconsciously perceive patterns and opportunities for improvement in his or her daily work life (Dutta \& Crossan, 2005). Once that individual interacts or shares those ideas with another, they enter the interpreting phase. This phase is about the final formalization of the idea to the individual and the explanation of the idea to others in the organization. Once multiple people in the organization begin changing their behavior to coincide with the new idea, integrating happens. In this phase, conversations and joint action are vital to making lasting change. The process will initially be informal, similar to a shared behavior, until institutionalizing happens. This final phase is the formal adoption and codification of the new process within the organization (Lawrence, Mauws, Dyck, \& Kleysen, 2005). Specific tasks and duties are assigned out and organizational changes are made to ensure the continuation of the new 
idea (Crossan et al., 1999). Consequently, if a leader can make change through individual action and throughout the entire organization, the 4I's are key.

Finally, this leads to the concept of Data Driven Decision Making. Marsh, Pane, and Hamilton (2006) made the connection between DDDM and Organizational Learning Theory. They discussed how DDDM within education is modeled on practices from industry, such as "total quality management, organizational learning, and continuous improvement (Marsh et al., 2006).”

\section{Data-Driven Decision Making}

The concept of data-driven decision-making (DDDM) within education stems from research by Popham et al. (1985). This research focused on the K-12 environment and how states began requiring the use of outcome data in school improvement planning and strategic planning (Popham, 1987; Popham et al., 1985). Additionally, the No Child Left Behind Act (NCLB, 2001) provided opportunities and incentives for educators to use data, mostly test scores, to secure funding and improvement for their schools. DDDM has been explored mostly within the business or private enterprise world, as education has been slower to adopt the use of statistics and data than the business world (Bontrager, 2004).

March, Pane, and Hamilton (2006) postulated how there are various types of data, and DDDM practitioner is to take the various types of data and turn them into "actionable knowledge." This knowledge informs various decisions, leading to the successful utilization of DDDM (Marsh et al.). The ability to turn the massive amount of data that an enrollment management (EM) office receives into actionable knowledge is a major reason DDDM was chosen in this inquiry. Today, there is more data available for college 
admissions offices than ever before, and personnel can become overwhelmed by the data. Being able to organize and utilize the data effectively directly impacts the success of an EM team (Natek \& Zwilling, 2014).

While DDDM may come from industry, the idea of using it in education is not new (Popham, 1987; Popham et al., 1985), with a myriad of reforms starting in the 1980s and peaking with the national No Child Left Behind Act of 2001 (NCLB, 2001). As Kowalski (2010) noted, critics of NCLB have often reacted negatively to the laws requirement for Data-Driven Decision Making, highlighting "they (educators) have argued that basing consequential decisions solely on research data is demeaning and precarious, demeaning because the wisdom of educators is devalued and precarious because research data is fallible (p. 3). This is one of the major issues with using data in education because some practitioners feel that their experience and anecdotal evidence is superior to hard data. The use of data in PK-12 education became a necessity for administrators (NCLB, 2001) and educators as they began to be held to standards of accountability (Lew \& Nelson, 2016). It has been only recently that the value of a college education has been held to a higher accountability (Webber, 2016; Williams, 2016).

Recent years have seen an explosion of research into how data can be used within offices of enrollment management to become more successful. As noted earlier, resources are becoming more and more limited within higher education, therefore requiring strategic enrollment managers to be precise in their efforts of data analyzation, which is an important tool (Cox et al., 2017; Picciano, 2012). This change in perspective and focus in the research to the use of data in enrollment management naturally led to an additional focus on technology and how it can be used. The research emphasizes that technology is 
key for offices of enrollment management that are attempting to become data driven, as the sheer volume of data both available and needed to be successful, requires the use of advanced technology such as robust Customer Relationship Management (CRM) systems, Student Information Systems (SIS), and data analytics tools (Attaran, Stark, \& Stotler, 2018; Hubbard, Datnow, \& Pruyn, 2014).

Furthermore, the previously mentioned changes in the higher education landscape, particularly when it comes to funding, makes the need for a shift toward datadriven decision making more necessary. This is especially true for public schools, as the sharpest decline in funding has been from state governments (Sav, 2016). As previously mentioned, the larger public schools have had to change their recruitment and strategic enrollment management efforts to mirror those of smaller private schools, namely focusing on recruiting on an individual basis (Bontrager, 2004). For small private schools, this did not require a large leap in technology, as the volume of students being recruited was small. For the average small to mid-size private school, there are a few hundred applications to manage, easily done with simple technol,ogy. However, for a mid-size to large public school there are thousands of applications with hundreds of thousands of "leads" to manage (IPEDS), requiring robust technology, which costs money (Sav, 2016).

Consequently, this need for a more efficient means in which to store and analyze data led to the most recent revolution regarding data-driven decision-making in higher education. The emergence and reliance on high-end customer relationship management systems or CRMs (Sutin, 2018). CRMs are databases that allow a user to store large amounts of data and create efficiencies in workflows. For example, the modern CRM 
allows offices of enrollment management to create communication plans, track student behavior, plan admission counselor travel schedules and communicate directly with students (Hwang \& Choi, 2019). The outcome of the system utilization is that large schools, recruiting to large volumes of students, can recruit students more individually without the tedious effort of historic individual recruiting. In the past, this individual recruitment would have required days of effort by an admissions counselor. Now, in minutes, a record can be created, and the transition to recruitment is seamless. This all leads to more efficiency in time and money, built on the framework of data-driven decision-making. The principles of DDDM are important at the initial stage of the CRM implementation. Offices of enrollment management use their available data in several ways. First, they use the data to know which students to focus on by utilizing predictive modeling. Second, data is used to know how to best communicate with students and the best times to implement those communications. Additionally, the data can be utilized to know how to best engage students in the recruitment process and how to help the student complete all the necessary paperwork to become a student. Consequentially, data is essential for enrollment managers to know how to best yield the student, or close the deal (Levin, 2015).

Upon completion of this review of literature, organizational learning theory, and data-driven decision-making emerged as the obvious frameworks in which to undertake this inquiry. As the focus of this research is on how EM offices adapt, learn, and grow into organizations that strategically use data, OLT provides a clear and accurate lens through which to examine the growth and change of an EM office. Specifically, viewing change as a type of renewal, provides a strong foundation, as evident in the literature, this 
type of learning process tends to be tumultuous and challenging (Crossan \& Berdrow, 2003). Furthermore, as the desired result of the learning process is an organization that embraces and strategically utilizes data, DDDM is a well-established model through which to approach this inquiry. The key factor being investigated is how an organization uses data strategically. Considering the literature, organizational learning theory and datadriven decision-making emerged as the conceptual frameworks to be utilized in this inquiry.

\section{Methodological Details}

To investigate how EM offices within two universities of varying demographics utilized OLT and DDDM to inform their practices, a parallel case study approach was selected for the design of the study. A parallel case study provides a broader detailed investigation of programs or processes and is also bound by time (Creswell, 2014). The research lends itself well to this approach as well as utilizing grounded theory, which uses the investigator(s) as the primary instrument of data collection analyzing a process or action from the view of the participants to form a general theory (Creswell, 2014). Data in grounded theory tends to come from interviews (Merriam \& Tisdell, 2016), which along with focus groups, will be the primary instruments of data gathering in this research. Interviews as a data-gathering tool are used primarily because grounded theory focuses on the view of the participants, and interviews are the stories of people, or for this research study, the participants (Seidman, 2013). While utilizing stories may not seem academic, Bertaux (1981) argued that utilizing interviews in social science research is vital as the subjects in social science are people with the ability to think and talk. These 
are key characteristics of subjects in social science and key differences between the subjects of social and natural or physical science.

The researchers conducted four focus groups. This is a valuable method of collecting data in a qualitative study due to its nature of being a socially constructed group of people with knowledge in the area being studied (Merriam \& Tisdell, 2016). As EM offices consist of a wide variety of employees, focus groups were deemed an important way to gather data so all voices can be heard. Furthermore, focus groups give people an opportunity to discuss things in an open, safe environment, which leads to honest and truthful data emerging (Krueger \& Casey, 2015).

\section{Setting}

The multi-case study consisted of two universities of different types to gain a broader perspective (Hancock \& Algozzine, 2015; G. Thomas, 2015). The Universities include a large, Division 1, Research 1 public university, and a mid-size private university. The sizes and descriptions come from the Carnegie Classification System (Research, 2017).

The first university was a large, public, high research/research one university in the southern United States. The size definition of a large university and the high research definition come from the Carnegie Classification System (Research, 2017), with large being 10,000 or more students. Current enrollment at the large institution included in this research is 37,010 (Facts, 2018). High Research/Research 1 University comes from the Carnegie Classification System, with 1 being a university with the Highest Research Activity. There are less than 120 of these in the country (Research, 2017). 
The university enrolls 37,010 students per year through a combination of undergraduate, graduate, law school, medical school, and nursing school (Facts, 2018). The university is diverse, with Hispanic students representing $27.8 \%$ of the undergraduate class, which qualifies the institution as a Hispanic Serving Institution (Facts, 2018; HACU, 2017). The undergraduate population accounts for 30,737, and the undergraduate enrollment office will be the focus of this research. The undergraduate enrollment office employs 55 full-time staff in recruiting, processing, and through the visitors center. The researcher identified the institution, through professional networking, as a university that has created their predictive model and has undergone significant change within the office of EM within the last ten years. This university will be an appropriate setting to investigate how the practices examined in this investigation progress from ideas to implementation since their senior leadership has been in place for roughly ten years. The current leadership was instrumental in the creation of their predictive model, and they fully subscribe to the ideas within organizational learning. However, a limitation to this setting is the institution resides in a state (one of only two states) that is currently growing in high school graduating students (Hussar \& Bailey, 2016) and one where money is less of an issue. Therefore, some of the processes they have implemented may not be replicable at smaller institutions. However, the knowledge gained from their experiences makes the inquiry valuable.

The second institution was a mid-size, private university in the upper mid-west, in a large urban center. Mid-size, or medium university, is defined by the Carnegie Classification System (Research, 2017), with the medium being 3,000 - 9,999. Current enrollment at the medium university is 7,266 through a combination of undergraduate, 
graduate, and law students. Undergraduate students make up 2,724 of the total population, and the undergraduate admissions office will be the focus of this inquiry. This campus is also diverse, specifically when it comes to international students. International students make up $21 \%$ of the undergraduate population, within 100 different countries represented. This institution is a high achieving technical school, with a heavy focus on engineering, science, and architecture, with an average incoming ACT of nearly 29. The undergraduate admissions office employs 22 full-time staff members through a combination of recruiting and processing. Similar to the other case study, through professional networking, the researcher identified the institution as one that has created their predictive model and has undergone change within the last five years. This university will be an appropriate setting to investigate how the practices examined in this investigation progress from ideas to implementation, as the senior leadership has been in place for roughly five years. The current leadership was instrumental in the creation of the predictive model and fully subscribed to the ideas within organizational learning.

Private schools have a history of being early adopters when it comes to strategic EM practices (Bontrager, 2004), making the perspective gained from this institution valuable. These types of schools are usually enrolling classes of less than five hundred, sometimes less than one hundred. To be successful, they should know as much as possible about their students and their likelihood to enroll. This type of institution and the knowledge gained from it is valuable, as large schools have to adapt to using this private school model of recruitment, on a larger scale. As such, the private schools have an advantage over public schools, as these universities have been utilizing these strategies for most of their existence (Han, 2014). Conversely, in general, these universities lack the 
scalability quantity gained from investigating larger schools; however, with this being a larger private school, scalability issues will be minimal.

\section{Interview Protocol}

Interviewed were eight participants at each institution and included the Director of Admission, the Vice President/Provost for EM, a mid-level assistant director, and an admissions representative. The interviews consisted of 10 questions, each focused on a specific element of the research questions. Questions will guide the subjects on explaining how their institution used organizational learning to improve their ability to recruit and retain students. Questions focused on the data-driven decision-making model and how that affects enrollment (Creswell, 2014) (see Appendix B).

Furthermore, as Merriam and Tisdell (2016) discussed, the interview questions were established based on the research questions. Since the researcher is an enrollment management professional, questions were piloted amongst internal EM personnel and adjusted accordingly. Questions were asked, answers were noted, and the information gained was documented. Questions were modified to allow more answers that are openended as well as ensuring all research questions were addressed in the interviews.

As outlined by Merriam and Tisdell (2016), the interviews were semi-structured, whereby the questions will be worded flexibly, leading to a more organic conversation. Specific information was targeted, but rather than asking more direct and structured questions; this approach allowed the researchers to respond to the situation and to any new ideas or relevant topics that emerge naturally from the situation.

Each participant was purposefully selected as they reflect the average person represented in an enrollment management office (Merriam \& Tisdell, 2016) and to gain 
information on specific research questions. The director of admission was chosen to provide overall guidance on the change process, as she was the direct change agent. Additionally, this person was chosen to provide insight into the large-scale workings of the office and how leadership directly affects organizational learning. The vice president/provost for EM was included to provide additional insight into overall effects on the office, as well as how the learning done by the admissions office has affected the university on a larger scale. The assistant director and admissions representative were chosen to provide extra insight into the leadership aspects of organizational learning, as well as the day-to-day ramifications of the change. These interviews were audiorecorded, and before beginning the audio recording, each participant will be read an informed consent form that has been approved by the University of Missouri IRB.

\section{Focus Group Protocol}

For the focus groups, two were conducted at each institution for a total of four focus groups. One included a mix of enrollment management staff not included in the interviews, and one consisted of current students. This allowed full perspective of the enrollment cycle, from both the institution side as well as the student being recruited side. Focus groups were between five and eight participants, as this is the ideal size for qualitative research (Krueger \& Casey, 2015). This allows all participants to share their viewpoints and feel equally included.

To ensure validity (Creswell, 2014), questions for the focus group were created using the researchers own background as an enrollment management professional and tested for validity. Questions were pilot-tested internally within an enrollment management office, both with professional staff and current student workers, to ensure 
they were understandable and provided answers to the relevant research questions and topics (Krueger \& Casey, 2015). Similarly to the interview questions, focus group questions were semi-structured, as outlined by Merriam and Tisdell (2016), meaning the questions will be worded flexibly, leading to a more robust conversation.

The focus group questions with the employees pertained to the perceived changes and successes, as discussed by those using the new models created by the senior administration. The employees were selected purposefully (Krueger \& Casey, 2015), as discussed previously. The focus group with the students discussed how this universities recruitment differed from others that the students interacted with, and what about this model made them choose that school (Creswell, 2014). Students were selected via organizational recruiting (Krueger \& Casey, 2015) meaning that volunteers from the current student body were accepted. In addition to the random student volunteers, current student workers within the admissions office also volunteered and were randomly selected from the pool of volunteers to join the focus groups as well. These focus group sessions were audio-recorded, and before beginning the audio recording, each participant signed an informed consent form.

\section{Document and Artifacts}

Documents and artifacts are key components in the data collection efforts of qualitative research (Merriam \& Tisdell, 2016). In general, documents are materials, while artifacts are things or objects that exist in the environment of the study (Merriam \& Tisdell, 2016). An important category of documents used in this study was records and statistical databases. In the field of education, public documents such as statistics and data are vital to telling the whole story, and specifically, things not observed (Merriam \& 
Tisdell, 2016). For this study, the researcher used data collected by the National Student Clearinghouse (NSC) as well as the Integrated Postsecondary Education Data System (IPEDS). This data set included specific information on the number of students who persist and graduate at both institutions and comparative data on a national level. Specific documents werel not be included as much as data from the sources mentioned above. The purpose of adding this national data was to validate enrollment data as presented by the university. The overriding theme of this research was how an enrollment office can be successful based on student growth. Data triangulation with internal, institutional data along with the national data assisted with validity (Creswell, 2014).

\section{Data Analysis Procedures}

Data analysis within qualitative research was both ongoing and all-encompassing (Creswell, 2014). The researchers analyzed data throughout the study, as opposed to gathering all the data and then analyzing at the end, as in quantitative research. Additionally, the analysis considered all aspects of the research and the interactions amongst the various parts. In other words, data does not live independently but is intertwined (Creswell, 2014). Furthermore, when utilizing a case study, the researcher must pay strict attention to data management, as multiple sources of sometimescontradictory data can lead to confusion and misinterpretation (Merriam \& Tisdell, 2016). Merriam and Tisdell (2016) similarly stated that to begin the data analysis in a case study, and all the evidence must be brought together to be analyzed, both as a whole and as independent parts.

After the transcription of the interviews and focus groups, in which the researcher used member-checking (Creswell, 2014), the researcher read the transcripts in their 
entirety to obtain a broad perspective of the participants' perception and code for themes (Merriam \& Tisdell, 2016). Coding here means organizing the data into segments that correlate to themes as they relate to the research questions (Creswell, 2014). Practices included but are not limited to segmenting sections of text from interviews and focus groups, highlighting, creating theme categories, and creating theme terms that correspond to research questions (Creswell, 2014). The researcher specifically examined topics that are based on past literature and experience, as well as topics that were not anticipated at the outset of the research. Once initial codes are created for topics, codes can then be grouped in a process known as axial coding (Merriam \& Tisdell, 2016). This coding is useful in that it goes beyond simple topic grouping and includes grouping based on meaning and thematic consistency.

\section{Limitations and Assumptions}

The major limitation of this study was the lack of accessibility to all students who could participate in the focus groups. The most effective way to gain insight into what practices of enrollment management impact a students decision to enroll at a university would be to directly interview the students. Hence, the entire purpose of interviews (Creswell, 2014). However, due to the difficulty of gaining access to current students, this study used a mix of current students and student workers in the admissions offices of both institutions. While this student perspective is valuable, most of these students will not be recently admitted, and they carried potential bias due to their employment within the offices of admissions. For a more inclusive focus group, participants would be from the general student population, and all would have been within one semester of being 
admitted. Therefore, all students interviewed would be recently recruited to the university.

In an ideal environment, this research would have included participation from a mid-sized, regional, public school, to include a more robust sampling of university types. Public institutions in the Midwest are encountering record budget cuts, drops in enrollment, and challenges from all sides as to their value (Calderon \& Mathies, 2013). These schools represent a large percentage of universities in the mid-west, and their perspective would be valuable. However, gaining access to these schools is difficult, as universities in the Midwest have strong competition with other, mid-west, regional, public institutions.

A final limitation to this study included the researchers connection to the universities and the leaders interviewed. The primary change agent and leader at both schools are former employers of the researcher, and the researcher worked for nearly seven years at the large university. Potential bias has framed the approach to the research, and consequentially, all data from interviews, focus groups, and document analysis will be vetted using member checking to ensure that personal bias does not affect the transcription of the sessions (Creswell, 2014).

\section{Design Controls}

The researcher utilized several controls to reduce bias in the research. Initially, the researcher used only semi-structured open-ended questions in the interview and focus group settings. This allows the participants to share opinions and views without being influenced by the researchers viewpoints (Merriam \& Tisdell, 2016). Coding of the data was done in a manner that was consistent with current practice and the established 
literature in the fields of higher education, enrollment management, data-driven decisionmaking, and organizational learning, including the use of axial coding (Creswell, 2014).

To this end, member checking allows bias to minimize by participants reviewing their responses and making changes to convey the correct information and tone (Creswell, 2014). While the researcher does have a personal connection to the leaders at the two institutions being examined, random sampling of focus group participants and interview participants (when possible), reduced the potential of bias.

\section{Presentation of the Data}

The research for this project was conducted on the campus of two universities. At each university, a senior leader was interviewed (a vice president and a vice provost, both of enrollment management). Additional interviews at each school consisted of an admissions director, an assistant director, and an admissions representative. Also, on both campuses, two focus groups were conducted, one of current students and one of other enrollment management leaders, for a total of four focus groups and eight interviews. The below chart will explain how each interview and focus group will be referenced in the discussion of the results:

\begin{tabular}{|l|l|}
\hline VP 1 & Vice President/Provost at school \# 1 \\
\hline VP 2 & Vice President/Provost at school \# 2 \\
\hline Director 1 & Director at school \# 1 \\
\hline Director 2 & Director at school \# 2 \\
\hline AD 1 & Assistant Director at school \# 1 \\
\hline AD 2 & Assistant Director at school \# 2 \\
\hline AC 1 & Admissions Counselor at school \# 1 \\
\hline
\end{tabular}




\begin{tabular}{|c|c|}
\hline AC 2 & Admissions Counselor at school \# 2 \\
\hline SFG 1 & Student Focus Group at school \# 1 \\
\hline Student Participant $1,2,3,4,5$ & Various student participants in the student focus group \\
\hline SFG 2 & Student Focus Group at school \# 2 \\
\hline Student Participant $1,2,3,4,5$ & Various student participants in the student focus group \\
\hline EMFG 1 & Enrollment Management focus group at school \# 1 \\
\hline EM Participant $1,2,3,4,5$ & Various EM professional in the EM focus group \\
\hline EMFG 2 & Enrollment Management focus group at school \# 2 \\
\hline EM Participant $1,2,3,4,5$ & Various EM professional in the EM focus group \\
\hline
\end{tabular}

Questions were designed by the researcher to assess if and how data was being used in admissions and enrollment management as a whole as well as if and how organizational learning was happening. Additional questions were asked to assess leadership and follower behaviors, cultural impact and importance, and the effectiveness of recruitment strategies developed by the enrollment management offices of each university. Interviews and focus groups were recorded and transcribed for ease of analysis. The transcripts were provided to the participants for member checking to ensure accuracy.

The analysis procedure addressed the four research questions as well as evaluating the results through the framework of organizational learning. The primary means of evaluation will be the life-cycle process assessment posited by Argote and Miron-Spektor (2011) in conjunction with the tacit to explicit knowledge conversion theory presented by Nonaka and Takeuchi (1995). 


\section{Research Question One}

How does the use of enrollment data for data-driven decision-making affect the organizational learning of the university, and what type of learning is derived?

At both institutions, it was clear that data is behind almost every decision made in the enrollment management offices. As VP 2 said, "data is the backbone of all decision making in our department." Director 2 backed up this idea, "we use data to make every single strategic decision as a management team as well as empower our admissions counselors to use data to determine what high schools they are going to visit." This theme of using data in both strategic planning and day-to-day operations was repeated at all levels at both institutions, in every interview. A direct example of this comes from AC 1, who outlines her planning process for fall recruitment season. She outlines how she starts with lists of her current class of students she is going to be focusing on, reports on the territory itself, the various high schools, relationships with counselors, what types of visits each high school offers, and internally created profiles using predictive modeling that allows her to strategically plan and organize her travel.

Research question one focuses on how the use of data-driven decision-making affects the organizations ability to learn. As one evaluates this through the lens of process assessment, the relationship becomes clear. Argote and Minon-Spektor (2011) argued that initially, experience is transformed to sharable knowledge. The recruitment profiles mentioned previously are one example of this idea. Using previous experience from the admissions and enrollment management team, the EM office has created a predictive model. This model is based on using experience with students and understanding how their behavior in the recruitment process will transform into enrollment behavior. For 
example, Director 2 explained that, through experience, they know that if a student engages in certain recruitment events or with certain marketing pieces or if they engage in certain ways with an admissions counselor, their likelihood to enroll goes up. This past information then informs future behavior, and the organization has learned what works and what does not.

The next phase of the process assessment involves taking that learned knowledge of the individual and sharing it organization wide. An example of this comes from the EMFG2. EM Participant 1 told me that through communication with the admissions team, they were recently able to re-evaluate their awarding process and be more efficient in the awarding of aid. In the middle of their awarding cycle for the previous enrollment year, the enrollment goals were changed. This led to many necessary conversations between admissions and financial aid so that they could meet those goals, which had changed not only in overall quantity of students desired but a change in the quality of the incoming class. The goal had been to maintain or grow slightly with a keen eye toward quality, or the credentials of the incoming class. In February the goal was changed to increase quantity dramatically and increase quality. Only through their collaborations and use of data on what types of awarding would most likely result in certain behaviors, were they able to achieve those goals. As EM Participant 1 said, "we created a model that allowed us to offer three thousand more scholarships than previously but at the same cost or even slightly less the previous years." By using the data and determining that smaller awards to more students would allow them grow as desired, they were able to increase the class without spending more money. 
This leads to the final aspect of the process assessment cycle, wherein the environment of sharing and learning becomes the culture. Nearly every interview and focus group discussed culture and the importance of it. Director 1 said it plainly, "Culture eats strategy for breakfast." When asked to explain, she explained "that you can have all the data in the world and create perfect strategy, but if you don't have a team to execute it, you won't be successful." This theme was repeated in the EM1 focus group who talked about the impact of their new leader and how the culture of open communication has been key to their success. EM1 Participant 1 noted that "Previously, we had been siloed and didn't understand how our work impacted enrollment management as a whole. By taking down those walls and allowing us all to see how everything works together, we all moved forward together." Another example of how the use of data can help build culture comes from AD 2. She talked about how when she first started; she was amazed at how much data was used in the decision-making and strategic planning processes. She said "More so than anywhere else I have worked, we used data for everything and I couldn't imagine how we could use data more." Now, after being there for almost three years, she reports, "We continue to make more and more decisions using data, and just when I think we can't find any more data points to use, we do and use them to successfully guide our strategic planning." It has now become so engrained in the culture, that the possibility of success without using data is hardly considered. What was once a place that VP 2 described as a place that "wasn't using data at all in my opinion, and was relying more on anecdotal evidence or doing what had always been done" is now so data-driven, employees cannot imagine a world without data. 
In conclusion, it is clear that utilizing data to enable data-driven decision making can and does lead to organizational learning. It is also clear that the resulting shifts in culture are vital to success. All participants interviewed and involved in the non-student focus groups noted the importance of culture. By creating a culture where ideas can be shared openly and effectively, meaning leaders listen to ideas and respect the experience of the members of the organization, organizational change can and does happen. The interesting component of this research question is that data alone is not enough, nor is well intentioned leadership. If an organization possesses solid data but has no leadership willing to create culture of communication and mutual respect, very little success will come from the data. An organization could create excellent strategy in this situation, but the carrying out of that strategy would falter. Conversely, if an organization has amazing culture they may be great at the closing the deal portion of recruitment, but their strategy would not be putting them in the best situations to be successful. A merger of the two is essential to success.

\section{Research Question Two}

How can an enrollment management office create a data-driven recruitment model through organizational learning and implement it successfully?

The answer to this question that emerges from the data is quite simple, in theory, less simple in practice. It is clear from these two universities that they have set up their offices along the path of the process assessment life-cycle model used in this research as an assessment tool. No one at either university said they did so intentionally, but it is clear from the evaluation process, that has indeed happened. Both universities created an environment in which individual learning can become organizational knowledge and 
practice, which in turn creates a culture of collaboration and communication. The results of this research and the impressive success these two vastly different universities have achieved, make it clear that following this model can lead to success. However, it is not as simple as knowing one path to success. There are many paths to success, as well as other universities and organizations that have started down this path and not been successful. In answering this question, the researcher will focus on specific practices and ideas that emerged from the research that can help other enrollment management offices achieve success.

A great example of this comes from AD 2. She explained a situation with an out of state recruiter, specifically focusing on California and the Phoenix area of Arizona. Initially, after reviewing the historic data, a new recruiter position was created to serve California and Arizona. Based on past engagement and enrollment, it was determined that the recruiter would spend the majority of their time in southern California and Phoenix, with little to no travel in Northern California. Marketing campaigns would go to all regions, but specific regional travel was focused as described. Over three years, enrollment grew significantly in southern California, grew slightly in northern California, and remained the same in Phoenix. It was then decided to stop traveling to Phoenix and travel more to northern California. After another three year time period, it was discovered that enrollment continued to grow significantly in southern California, was now growing significantly in northern California, and was remaining the same in Phoenix. The organization learned that on the ground, recruitment efforts were not as important in the Phoenix market, and they could re-allocate the same amount of resources (travel to northern California instead of Arizona) and achieve greater results. Only through the 
process of the recruiter sharing their experiences, which in turn became institutional knowledge was this change possible. This is a direct example of creating a data-driven recruitment plan through the organizational learning process.

Another example comes in the form of scholarship strategy. VP 2 told me about a situation with their scholarship strategy this year. They know that their average SAT score is an 1169, and it is around that score that they yield the most students. As they work to increase their academic quality of their incoming class, it would be easy to offer large awards to the students that score $1500+$. However, from the use of data, they know those students are not likely to enroll, no matter how much money is offered. Therefore, they decided to focus on students in the 1200-1300 range, and they revised their top scholarship program accordingly. Instead of giving a larger amount to fewer very high scoring students, who yield at a low rate, they gave a lower amount to the 1200-1300 range students and doubled their yield by spending the same to a little less in scholarship dollars. This is another example of the organization learning through data and implementing a successful change.

Furthermore, when considering this question and how an organization can learn and adapt using data, it is also important to look at different student populations. In this case, specifically comparing how the use of data can affect transfer students compared to first time first-year students. Historically, data on transfer students has been lacking compared to what is available for first-year students. This is due to several factors, the largest one being that there are repositories of data for freshman, such as the ACT or SAT, while there is nothing like that for transfer. However, in this research, it seems clear that using data-driven decision-making is just as valuable for transfer students as it is for 
freshman; it is just harder to get that data. Nearly every person I interviewed expressed a desire to have more data on transfer students. Director 2 mentioned that he would like to have more data on transfer students to nail down where they are currently located and when they are interested in transferring as well as opening better channels of communication so that customized recruitment plans could be created. In the EMFG 2, participant 2 talked about how personalized and customized messaging is for freshman as they have so much data on them. For transfer students, that is not the case, as once they fall out of the freshman funnel, unless they are actively engaging with the university, it is hard to keep track of them and their needs. Director 1 also weighed in on transfer students, re-emphasizing the desire to use a data-driven approach, but noting the lack of data. She says "there is a general lack of data on this side and it is very messy as the data we do have is not under one place."

Furthermore, the lack of a concentrated area in which to preform search, meaning a platform in which a university can purchase transfer prospects, make recruiting transfer students difficult. Director 1 also mentioned the changing landscape of transfer students for her school specifically, talking about how they are getting more and more students transferring from 4 year schools, not just community colleges. This opens a whole new realm of issues, as you have to figure out what caused their poor experience and how you can avoid that upon transfer. In general, there is a definite desire to use a data-driven model for transfers, and, when available, it is successful. However, the overall lack of available transfer data makes this difficult and is a needed change in the industry.

In conclusion, it is clear that quality, trustable data is key to creating an environment of data-driven decision-making. Nearly all participants discussed how one of the biggest barriers to relying on data is getting clean data and how that process can take years. One of 
the biggest problems is getting consistent data across a large organization like a college or university. However, it is also clear that once an enrollment management team has data they trust, the strategic benefits are enormous. Data-driven enrollment managers calculate return on investment on all of their recruitment efforts, which is vital in this climate of reduced budgets. There are numerous examples of how data allows a team to do more with less, which is a key finding and a strong argument for the necessity of data-driven decision making in enrollment management.

\section{Research Question Three}

What impact does the predictive model have on recruitment and retention, and is that impact equal across different student populations?

Both universities utilize an internally created predictive model in their recruitment process. The predictive models created by these two universities take into account demographic data on the students as well as engagement activities. For example, the more a student interacts with the university in the recruitment process (coming to events, responding to emails, answering phone calls, etc...), the more likely the student is to enroll. This is calculated based on noting interactions over many years of recruitment and then following up with those students to see if they enrolled. Over time a pattern emerges that can be then used to create a mathematic probability or predictive value score. These scores are used by the university in a variety of ways, many of which were mentioned in the research.

An important point to make at the outset of the discussion on predictive modeling is the importance of clean data and having people in place to interpret the data. Both VP1 and VP2 mentioned this repeatedly. Both of them specifically described how they 
revamped their data teams, going through a process of hiring a strong data analysis team and making sure the data being pulled in admissions aligned with the data being pulled from institutional research. VP 2 noted, "What gets measured is what gets done," about the importance of tracking items that you wish to move the needle on, and the only way you can do this is to have good data.

In the EMFG 1, participant 1 and participant 2 talked about using the predictive model as an enrollment management team. They talked about how that was one of the biggest changes with the current leader. Before that leader, admissions had a model score, but it was not shared across the EM team. Now, they all have access to it and use it cohesively as one unit. For example, admissions uses it to develop a specific recruitment strategy for a student, financial aid will use it to help determine scholarship strategy, and marketing uses it to inform communication flow. AC 1 noted how she uses the predictive model to set her entire recruitment strategy, focusing on those students who are most likely to enroll and allocating her time accordingly. This sentiment was echoed by AC 2 as well, noting "everything I do to plan travel starts with historic data and the predictive model."

Another example of the use of predictive modeling comes from VP 1 and ties in the idea of retention. As mentioned previously, the goal is to recruit retainable students, not just admissible students. VP 1 told me about some data analysis he does in regards to whether a student will persist or not return, and he has it narrowed down to one variable, how they performed on a math placement exam. As he told me "if a student doesn't pass the math placement exam and goes into a remedial class, they have about a $70 \%$ chance of continuing. If they pass and go into Calculus immediately, retention is about $80 \%$. 
Furthermore, if they have a high grade in the remedial course and continue, they are up to $90 \%$, but if they get a low grade in the remedial it falls to $50 \%$." When he first looked at the data he said "I laughed and told the math faculty we just needed to get rid of remedial math." Obviously that is not the answer, but he talked about the danger of incorrect interpretations of data and how they can use this data to strengthen their remedial math program to better prepare students for calculus and beyond. His point is important here as it was echoed across most of the interviews and focus groups, interpretation matters. It is not enough to have clean data; you must have a person or team to analyze it as well as develop strategy.

In conclusion, predictive modeling is important and helpful to enrollment managers when used properly and effectively. This is one area where it would be extremely possible to get bogged down in too much data if a solid strategy was not in place to process, analyze, and strategize using the predictive model. Both of these schools do a great job in gathering clean, usable, and replicable data. Both schools have teams in place with years of experience in creating the models as well as developing strategy from the predictive models. In this case, it is important to note that if a school does not have such teams in place, the use of a predictive model could be of little to no benefit and possibly even harmful. If a school were to collect the wrong data or make incorrect strategic decisions from incomplete data, recruitment strategy could be skewed in a way that would not have happened without the use of the model. As with all tools in an enrollment managers toolbox, this one especially must be used with experience and practice. 


\section{Research Question Four}

Do institutions that have successful enrollment management offices rely solely on datadriven decision-making, or are there elements of evidence-based practice involved?

For as long as enrollment management has been around, it has existed on a spectrum with art on one end and science on the other. In recent years, with more research focused on understanding enrollment management, more formal training for those involved in the field, and more professional development and sharing of ideas available, the field has moved closer to the science end of the spectrum. One of the results that surprised me the most from this research was how much anecdotal evidence or elements of evidence-based practice remain in effect at these schools. Nearly everyone I spoke with mentioned how the relationship side of recruitment is still an important and vital factor and can not be entirely replaced with data.

VP 1 talked to me about how important it is to know the students and know what matters to them from both a recruitment and retention standpoint. He also talked about how the student experience has become more important during his time in enrollment management. "It's almost like their experience is what matters, not just programs, but student satisfaction... knowing that if a student does certain things that will lead to more happiness, more student success, and ultimately more retention.”

The idea of feel and the use of evidence-based practice was clear when talking to the student focus groups. Over and over, I heard about "feel" and the "visit experience." SFG 1 participant 1 told me "I came to campus for a visit and fell in love with the students, how they explained their programs and how they felt about campus and the whole campus life." This was particularly interesting as admissions offices are more and 
more turning to utilizing current students in their recruitment practices, not just admissions, and other campus professionals. SFG 2 participant 1 said the same thing "I came for an overnight campus visit and fell in love. As an out of state student I didn't want to leave my family, but I didn't have much choice after my visit." This also reiterates the power of the campus visit, which is a known commodity among admission professionals. Perhaps the most powerful statement came from SFG 2 participant 2 who summed up her recruitment experience like this "every other school I visited made me feel like I would be lucky to go there, this school made me feel like they felt lucky to have me." This idea of creating a family was indicated as being as equally or in some cases more important than even financial considerations. SFG1 participant 2 told me "I had three offers, one in state and two out of state. I visited all three, and even though this school cost a little more, after all scholarships, I just felt at home here and couldn't see myself at the other two schools."

This ties in to evidence based practice, as there is no data on feel and comfort. However, experienced and creative enrollment managers can put some data behind this idea. While one can not create an ideal visit experience that is custom to every student, an enrollment manger could survey and talk to prospective and current students to see what matters most to them. Additionally, there are companies that have begun doing market research and creating mindset or profiles on prospective students that can help universities create even more customized marketing based on the specific interests of students. A great example of this comes from Director 1 and AC 1. They both told me about a relatively new initiative in their office that they call "passion projects." In short, the director has allowed each admissions counselor to pick one group of students that is 
important to them to focus on, on top of their assigned territory. For example, AC 1 was a first generation student, so she chose to work with prospective students who are first generation. A purely data-driven approach would have broken up the territories based on demographics, and a specific counselor would work with all students in a specific territory. While they use that approach for general recruitment, for the passion projects a counselor gets to focus on a group they are passionate about. This leads to an amazing experience for the student and is personally rewarding for the admissions counselor. AC 1 called it "a non-monetary bonus," a job perk that she gets just from doing her job. That type of approach is a definite mix of data-driven decision making and evidence based practice. One thing is clear from the research; data alone is not enough. A school can know everything possible about a student, deliver a timely and impressive financial aid and scholarship award, have top ranked academic and co-curricular programs, and still miss out on students. The campus visit matters, as does every interaction a student has with a given university.

In conclusion, to be a successful enrollment manager, one must employ elements of both data-driven decision-making and evidence based practice. The student experience still matters a great deal, and while one can customize this based on data to some extent, the execution and how you make a student feel will ultimately be what seals the deal. A data driven recruitment plan will not be successful if you do not have the right people in place to genuinely care about the incoming students. Conversely, having people who care about the prospective students but do not know where or how to recruit effectively, will also not lead to success. The successful enrolment management office of the future will rely on data-driven decision making informing the practices of a well-intentioned 
recruitment team who genuinely cares about the needs of incoming students and who can successfully communicate that care by creating a family type environment where the student feels connected and wanted. In short, data informs behavior, and the environment and genuineness matters. Art and science blended for maximum performance and success.

\section{Conclusions}

From this research, it appears that data-driven decision-making can be an effective means to create a successful enrollment management office. Through a practice of data-driven decision-making, organizational learning can happen, which will allow an organization to maintain success and build a culture that sustains that success. Three key themes emerged from the research that will help to inform best practices for other colleges and universities to implement ideas from this research.

First, to have an office that utilizes data-driven decision-making, an organization must have good data. Before a leader attempts to implement the practices of data-driven decision-making, the data must be clean, replicable, and usable. This is especially important if a leader is attempting to create change and move an organization to a more data-driven approach. As VP 2 noted, when she first began, the campus community was skeptical of data coming out of admissions, as it had been incorrect for years. She first began the process by cleaning all of their data and then working with others on campus to confirm their data, including brining in institutional research, so admissions data matched data at a university level. This gave admissions credibility across campus when she began presenting ideas and changes to a more data-driven approach. Furthermore, a proper team must be in place to analyze the data and build successful strategy from it. Having clean 
data is a key first step; however an organization needs the data analytics to take full advantage and become data-driven decision makers.

The second key theme that arose from the data relates to culture. Through a process of organizational learning, a strong culture can emerge. If a leader sets the culture to be one that is data-driven, the leader must be willing to listen to and learn from the others on the team. This creates an environment of sharing and communication, which will lead to all members of the organization feel comfortable sharing their experiences, which equals more data. This culture of openness and team first are key to success for any enrollment management office, as this translates to the student experience. If an office is plagued with negative culture, that will reflect in the recruitment process. As noted, students must feel comfortable and like they fit on a campus, and negative internal culture inevitably leads to a negative perception from the prospective students. The best strategy in the world will not work with negative culture. A great example of this goes back to the passion projects mentioned by Director 1 and AC 1. They have set this culture in their office where data is vital but just as vital is finding things you are passionate about and working those as well. By allowing the counselors to engage in work related activities that are connected to groups or ideas they are passionate about, work seems less like work, they have grown in enrollment, counselors report greater job satisfaction, and culture is created and improved.

The third and final theme that emerged from the research has to do with the idea of enrollment management being a mix of art and science. Historically, enrollment management was mostly art. There has not always been the large amounts of data available on students that there is today, and there was little to no formal training on how 
to be an enrollment manager. In 2018, the University of Southern California created the first Masters Degree in Enrollment Management. There are more and more professional development opportunities available for enrollment managers. There is much more data available to use in the recruitment process. However, even with all of that being true, there is still an element of art to the job of enrollment manager. The "feel" of a campus and a visit experience still matters a great deal to students. By utilizing data on what matters to students, an enrollment manager can get some grasp of an idea on how to create an excellent student experience. However, every student is different, and what they want out of their recruitment experience is different. The successful enrollment manager must create a team that can respond to these difference in a consistent and genuine manner. The entire campus must be on board, as well. If the student has a great experience with admissions, but then a different department on campus or an academic unit creates a negative experience, which is what the student will remember. The successful enrollment manger is a bridge-builder across a university who can successfully communicate the idea that recruitment is a campus wide job, not just a job for admissions. 


\section{Journal Article References}

Adelman, C. (2006). The toolbox revisited: Paths to degree completion from high school through college. Washington, DC.

Agarwal, R., \& Helfat, C. E. (2009). Strategic renewal of organizations. Organization Science, 20(2), 281-293.

An, B. P., \& Sorensen, K. N. (2017). Family stucture changes during high school and college selectivity. Research in Higher Education, 1-28.

Antons, C. M., \& Maltz, E. N. (2006). Expanding the role of institutional research at small private universities: A case study in enrollment management using data mining. New Directions for Institutional Research, 2006(131), 69-81.

Argote, L., \& Miron-Spektor, E. (2011). Organizational learning: From experience to knowledge. Organization science, 22(5), 1123-1137.

Attaran, M., Stark, J., \& Stotler, D. (2018). Opportunities and challenges for big data analytics in US higher education: A conceptual model for implementation. Industry and Higher Education, 32(3), 169-182.

Attewell, P. (1992). Technology diffusion and organizational learning: The case of business computing. Organization Science, 3(1), 1-19.

Baden-Fuller, C., \& Volberda, H. W. (1997). Strategic renewal: How large complex organizations prepare for the future. International Studies of Management \& Organization, 27(2), 95-120.

Baker, V. L., \& Baldwin, R. G. (2015). A case study of liberal arts colleges in the $21 \mathrm{st}$ century: Understanding organizational change and evolution in higher education. Innovative Higher Education, 40(3), 247-261.

Barton, A. L., \& Hirsch, J. K. (2016). Permissive parenting and mental health in college students: Mediating effects of academic entitlement. 64(1), 1-8.

Baum, S., Kurose, C., \& McPherson, M. (2013). An overview of American higher education. The Future of Children, 23(1), 17-39.

Bean, J., \& Hossler, D. (1990). The strategic management of college enrollments. San Franciso, CA: Jossey-Bass.

Bell, B. J., Gass, M. A., Nafziger, C. S., \& Starbuck, J. D. (2014). The state of knowledge of outdoor orientation programs: Current practices, research, and theory. 37(1), $31-45$. 
Berry, L. L. (1995). Relationship marketing of services-growing interest, emerging perspectives. Journal of the Academy of marketing science, 23(4), 236-245.

Bertaux, D. (1981). Bibliography and society: The life history approach in the social sciences. Thousand Oaks, CA: Sage.

Biesta, G. (2007). Why "what works" won't work: Evidence-based practice and the democratic deficit in educational research. Educational Theory, 57(1), 1-22.

Bolman, L. G., \& Deal, T. E. (2013). Reframing organizations: Artistry, choice, and leadership. San Francisco, CA: John Wiley \& Sons.

Bontrager, B. (2004). Strategic enrollment management: Core strategies and best practices. College and University, 79(4), 9.

Bouwma-Gearhart, J., \& Collins, J. (2015). What we know about data-driven decision making in higher education: Informing educational policy and practice. Paper presented at the Proceedings of International Academic Conferences.

Bowen, W. G., Bok, D., \& Burkhart, G. (1998). A report card on diversity: lessons for business from higher education. Harvard Business Review, 77(1), 138-149.

Brotherton, P. (2001). It takes a campus to graduate a student: A look at seven academic retention programs and what makes them effective. Diverse Issues in Higher Education, 18(18), 34.

Brown, M. G., Wohn, D. Y., \& Ellison, N. (2016). Without a map: College access and the online practices of youth from low-income communities. Computers \& Education, 92, 104-116.

Cabrera, A. F., Nora, A., \& Castaneda, M. B. (1993). College persistence: Structural equations modeling test of an integrated model of student retention. The Journal of Higher Education, 64(2), 123-139.

Calderon, A., \& Mathies, C. (2013). Institutional research in the future: Challenges within higher education and the need for excellence in professional practice. New Directions for Institutional Research, 2013(157), 77-90.

Cangelosi, V. E., \& Dill, W. R. (1965). Organizational learning: Observations toward a theory. Administrative Sciences Quarterly, 175-203.

Cegler, T. D. (2012). Targeted recruitment of GLBT students by colleges and universities. Journal of College Admission, 215, 18-23.

Chapman, R. (1984). Toward a theory of college choice: A model of college search and choice behavior. Alberta, Canada: University of Alberta. 
Chapman, R. L., Kennedy, J. L., Newell, A., \& Biel, W. C. (1959). The systems research laboratory's air defense experiments. Management Science, 5(3), 250-269.

Chart, T. O. (2018). Texas Tech University Organizational Chart. Retrieved from http://www.ttu.edu/administration/president/org-chart_10-3.pdf

Chiu, J., Economos, J., Markson, C., Raicovi, V., Howell, C., Morote, E.-S., \& Inserra, A. (2016). Which matters most? Perceptions of family income or parental education on academic achievement. New York Journal of Student Affairs, 16(2), 3-16.

Chiva, R. (2017). The learning organization and the level of consciousness. The Learning Organization, 24(3).

Choo, C. W. (1996). The knowing organization: How organizations use information to construct meaning, create knowledge and make decisions. International Journal of Information Management, 16(5), 329-340.

Cook, S. D., \& Yanow, D. (1993). Culture and organizational learning. Journal of Management Inquiry, 2(4), 373-390.

Coopey, J. (1995). The learning organization, power, politics and ideology introduction. Management \& Learning, 26(2), 193-213.

Cox, B. E., Reason, R. D., Tobolowsky, B. F., Brower, R. L., Patterson, S., Luczyk, S., \& Roberts, K. (2017). Lip service or actionable insights? Linking student experiences to institutional assesment and data-driven decision making in higher education. The Journal of Higher Education, 88(6), 835-862.

Creswell, J. W. (2014). Research Design: Qualitative, quantitative, and mixed method approaches. Thousand Oaks, CA: Sage Publications.

Crossan, M. M., \& Berdrow, I. (2003). Organizational learning and strategic renewal. Strategic Management Journal, 24(11), 1087-1105.

Crossan, M. M., Lane, H. W., \& White, R. E. (1999). An organizational learning framework: From intuition to institution. Academy of Management Review, 24(3), 522-537.

Cunningham, A. (2014, March 12, 2014). Tech programs ranked in Top 100 in United States. The Daily Toreador.

Cyert, R. M., \& March, J. G. (1963). A behavioral theory of the firm. Englewood Cliffs, NJ: Prentice-Hall. 
Daniel, B. (2015). Big data and analytics in higher education: Opportunities and challenges. British Journal of Educational Technology, 46(5), 904-920.

Davidson, C., \& Wilson, K. (2013). Reassessing Tinto's concepts of social and academic integration in student retention. Journal of College Student Retention: Research, Theory \& Practice, 15(3), 329-346.

DeShields Jr, O. W., Kara, A., \& Kaynak, E. (2005). Determinants of business student satisfaction and retention in higher education: applying Herzberg's two-factor theory. International journal of educational management, 19(2), 128-139.

DesJardins, S. L. (2002). An analytic strategy to assist institutional recruitment and marketing efforts. Research in Higher Education, 43(5), 531-553.

DesJardins, S. L., Ahlburg, D. A., \& McCall, B. P. (2006). An integrated model of application, admission, enrollment, and financial aid. The Journal of Higher Education, 77(3), 381-429.

DesJardins, S. L., Dundar, H., \& Hendel, D. D. (1999). Modeling the college application decision process in a land-grant university. Economics of Education Review, 18(1), 117-132.

Dodgson, M. (1991). Technology learning, technology strategy and competitive pressures. British Journal of Management, 2(3), 133-149.

Dolence, M. G. (1998). Strategic enrollment management. Hand-book for the College Admissions Profession, 71-91.

Drazin, R., Glynn, M. A., \& Kazanijan, R. K. (1999). Multilevel theorizing about creativity in organizations: A sensemaking perspective. Academy of Management Review, 24(2), 286-307.

Duncan, R. B. (1974). Modifications in decision structure in adapting to the environment: Some implications for organizational learning. Decision Sciences, 5(4), 705-725.

Dutta, D. K., \& Crossan, M. M. (2005). The nature of entrepreneurial opportunities: Understanding the process using the 4I organizational learning framework. Entrepreneurship Theory and Practice, 29(4), 425-449.

Education, D. o. (2008). Higher Education Act. Retrieved from https://www.hacu.net/hacu/Higher_Education_Act_(HEA).asp

Ehrenberg, R. G., Zhang, L., \& Levin, J. (2005). Crafting a class: The trade off between merit scholarships and enrolling lower-income students. The Review of Higher Education, 29(2), 195-212. 
Elliott, K. M., \& Healy, M. A. (2001). Key factors influencing student satisfaction related to recruitment and retention. Journal for Marketing for Higher Education, 10(4), $1-11$.

Erdem, M., \& Ucar, I. H. (2013). Learning organization perceptions in elementary education in terms of teachers and the effect of learning organization on organizational commitment. Educational Sciences: Theory and Practice, 13(3), 1527-1534.

Eskreis-Winkler, L., Shulman, E. P., Beal, S. A., \& Duckworth, A. L. (2014). The grit effect: Predicting retention in the military, the workplace, school and marriage. Frontiers in Psychology, 5.

Facts, T. Q. (2018). Quick Facts. Retrieved from http://www.ttu.edu/about/

Ferris, E., Finster, M., \& McDonald, D. (2004). Academic fit of student-athletes: An analysis of NCAA division IA graduation rates. Research in Higher Education, $45(6), 555-575$.

Foote, S. M., Kranzow, J., \& Hinkle, S. E. (2015). Focusing on the forgotten: An examination of the influences and innovative practices that affect community college transfer student success. In Examining the Impact of Community Colleges on the Global Workforce (pp. 94-124): IGI Global.

Ford, W. G. (2011). Evaluating the effectiveness of college web sites for prospective students. Journal of College Admission, 212, 26-31.

Francis, D. E. (2014). Lean and the learning organization in higher education. Canadian Journal of Educational Administration and Policy, 157, 1-23.

Freeman, K. (1997). Increasing African Americans' participation in higher education: African American high-school students' perspectives. Journal of Higher education, 68(5), 523-550.

Freitas, S. I., Morgan, J., \& Gibson, D. (2015). Will MOOCs transform learning and teaching in higher education? Engagement and course retention in online learning provision. British Journal of Educational Technology, 46(3), 455-471.

Fuller, B., Wright, J., Gesicki, K., \& Kang, E. (2007). Gauging growth: How to judge no child left behind? Educational Researcher, 36(5), 268-278.

Gikas, J., \& Grant, M. M. (2013). Mobile computing devices in higher education: Student perspectives on learning with cellphones, smartphones \& social media. The Internet and Higher Education, 19, 18-26. 
Goenner, C. F., \& Pauls, K. (2006). A predictive model of inquiry to enrollment. Research in Higher Education, 47(8), 935-956.

Goldstein, P. J., \& Katz, R. N. (2005). Academic Analytics: The uses of management information and technology in higher education. Washington D.C.: Educause Center for Applied Research.

Greene, J. P., \& Forster, G. (2003). Public high school graduation and college readiness rates in the United States. Education Working Paper No. 3. Center for Civic Innovation.

Greenleaf, R. K. (1970). The Servant as Leader. The Robert K. Greenleaf Center, Indianapolis, IN.

Guinot, J., Chiva, R., \& Mallén, F. (2016). Linking altruism and organizational learning capability: A study from excellent human resources management organizations in Spain. Journal of Business Ethics, 138(2), 349-364.

Gulick, L., \& Urwick, L. (Eds.). (1937). Papers on the science of administration. New York, NY: Columbia University Press.

HACU. (2017). Hispanic Serving Institutions Defintions. Retrieved from https://www.hacu.net/hacu/HSI_Definition1.asp

Han, P. (2014). A literature review on college choice and marketing strategies for recruitment. Family and Consumer Sciences Research Journal, 43(2), 120-130.

Hancock, D. R., \& Algozzine, B. (2015). Doing case study research: A practical guide for beginning researchers. New York, NY: Teachers College Press.

Hanushek, E., \& Lindseth, A. (2009). Performance-based funding. Education Week, 28, 28.

Hill, C. B., \& Winston, G. C. (2010). Low-income students and highly selective private colleges: Geography, searching and recruiting. Economics of Education Review, 29(4), 495-503.

Hoppes, C. R., \& Holley, K. A. (2014). Organizational trust in times of challenge: The impact on faculty and administrators. Innovative Higher Education, 39(3), 201216.

Hora, M. T., Bouwma-Gearhart, J., \& Park, H. J. (2017). Data driven decision-making in the era of accountability: Fostering faculty data cultures for learning. The Review of Higher Education, 40(3), 391-426. 
Hossler, D., \& Gallagher, K. S. (1987). Studying student college choice: A three-phase model and the implications for policymakers. College and University, 62(3), 207221.

Hossler, D., \& Kalsbeek, D. (2013). Enrollment management and managing enrollments: Revisiting the context for institutional strategy. Strategic Enrollment Management Quarterly, 1(1), 5-25.

Hotho, J. J., Lyles, M. A., \& Easterby-Smith, M. (2015). The mutual impact of global strategy and organizational themes and future directions. Global Strategy Journal, $5,85-112$.

Hotho, J. J., Saka-Helmhout, A., \& Becker-Ritterspach, F. (2014). Bringing context and structure back into situated learning. Management learning, 45(1), 57-80.

Hubbard, L., Datnow, A., \& Pruyn, L. (2014). Multiple initiatives, multiple challenges: The promise and pitfalls of implementing data. Studies in Educational Evaluation, $42,54-62$.

Hult, G. T. M., Mena, J. A., Ferrell, O. C., \& Ferrell, L. (2011). Stakeholder marketing: A definition and conceptual framework. AMS Review, 1(1), 44-65.

Hurst, D. K. (1995). Crisis \& renewal: Harvard Business School Press Boston, MA.

Hussar, W. J., \& Bailey, T. M. (2016). Projections of education statistics to 2023. NCES 2015-073. National Center for Education Statistics, 42, 1-157.

Hwang, Y.-S., \& Choi, Y. K. (2019). Higher education service quality and student satisfaction, institutional image, and behavioral intention. Social Behavior and Personality: An International Journal, 47(2), 1-12.

IIT. (2018). Quick Facts. Retrieved from https://web.iit.edu/about/quick-facts

IPEDS. IES/NCES National Center for Education Statistics. Retrieved from https://nces.ed.gov/ipeds/datacenter/

Irlbeck, E., Adams, S., Akers, C., Burris, S., \& Jones, S. (2014). First generation college students: Motivations and support systems. Journal for Agricultural Education, 55(2), 154-166.

Isaacs, M. L. (2003). Data-driven decision making: The engine of accountability. Professional School Counseling, 6(4), 288-295.

Jackson, G. A. (1982). Public efficiency and private choice in higher education. Educational Evaluation and Policy Analysis, 4(2), 237-247. 
Jelinek, M. (1979). Institutionalizing innovation: A study of organizational learning systems: Greenwood.

John, E. P. S. (1990). Price response in enrollment decisions: An analysis of the high school and beyond sophomore cohort. Research in Higher Education, 31(2), 161176.

Jose, M., Kurian, P. S., \& Biju, V. (2016). Progression analysis of students in a higher education institution using big data open source predictive modeling tool. Paper presented at the Big Data and Smart City (ICBDSC), 2016 3rd MEC International Conference.

Jyoti, J., \& Bhau, S. (2016). Transformational leadership and job performance: A study of higher education. Journal of Services Research, 15(2), 77-110.

Kabakchieva, D. (2013). Predicting student performance by using data mining methods for classification. Cybernetics \& Information Technologies, 13(1), 61-72.

Kaemar, M., Harris, K., Andrews, M., \& Tepper, B. J. (2013). Ethical leadership and subordinate outcomes: The mediating role of organizational politics and the moderating role of political skill. Journal of Business Ethics, 115, 33-44.

Kansal, A. K., \& Kaur, H. (2016). A study of career orientation of female college students in relation to parental education and employment status. International Education and Research Journal, 2(9).

Kao, G., \& Thompson, J. S. (2003). Racial and ethnic stratification in educational achievement and attainment. Annual review of sociology, 29(1), 417-442.

Kaufman, T. E., Graham, C. R., Picciano, A. G., Popham, J. A., \& Wiley, D. (2014). Data-driven decision making in the K-12 classroom. In Handbook of Research on Educational Communications \& Technology (pp. 337-346). New York, NY: Springer Press.

Kemerer, F. R., Baldridge, J. V., \& Green, K. C. (1982). Strategies for effective enrollment management: Amer Assn of State Colleges \&.

Kowalski, T. (2010). Handbook of Data-Based Decision Making in Education. New York, NY: Routledge Press.

Kretchmar, J., \& Memory, A. (2010). One college's journey into the unconscious mind of its prospective students: How a new research methodology is helping us recruit. Journal of College Admission, 207, 8-15.

Krueger, R. A., \& Casey, M. A. (2015). Focus groups: A practical guide for applied research: Sage publications. 
Kuh, G. D., Kinzie, J., Schuh, J. H., \& Whitt, E. J. (2011). Student success in college: Creating conditions that matter. San Franciso, CA: John Wiley \& Sons.

Lambert, L. S., Tepper, B. J., Carr, J. C., Holt, D. T., \& Barelka, A. J. (2012). Forgotten but not gone: An examination of fit between leader consideration and initiating structure needed and received. Journal of Applied Psychology, 97(5), 913.

Langley, A., Smallman, C., Tsoukas, H., \& Van de Ven, A. H. (2013). Process studies of change in organization and management: Unveiling temporality, activity, and flow. Academy of Management Journal, 56(1), 1-13.

Langston, R., \& Loreto, D. (2017). Seamless integration of predictive analytics and CRM within an undergraduate admissions recruitment and marketing plan. Strategic Enrollment Management Quarterly, 4(4), 161-172.

Lawrence, T. B., Mauws, M. K., Dyck, B., \& Kleysen, R. F. (2005). The politics of organizational learning: Integrating power into the 4I framework. Academy of Management Review, 30(1), 180-191.

Le, H., Robbins, S. B., \& Westrick, P. (2014). Predicting student enrollment and persistence in college STEM fields using an expanded PE fit framework: A largescale multilevel study. Journal of Applied Psychology, 99(5), 915.

Leroy, H., Anseel, F., Gardner, W. L., \& Sels, L. (2015). Authentic leadership, authentic followership, basic need satisfaction, and work role performance: A cross-level study. Journal of Management, 41(6), 1677-1697.

Levant, R., \& Silverstein, L. (2005). Gender is neglected in both evidence-based practices and "treatment as usual". Washington D.C.: APA Books.

Levi, D. (2014). Group dynamics for teams. New York, NY: Sage Publications.

Levin, R. (2015). Strategic Alignment: Recruiting students in a highly decentralized environment. College and University, 91(1), 47-54.

Levine, A., \& Dean, D. R. (2013). It's only technology if it happens after you are born. Journal of College Admission, 220, 6-12.

Levitt, B., \& March, J. G. (1988). Organizational learning. Annual Review for Sociology, 14(1), 319-338.

Lew, M. M., \& Nelson, R. F. (2016). New teachers' challenges: how culturally responsive teaching, classroom management, \& assessment literacy are intertwined. Multicultural Education, 23(3/4), 7. 
Lile, J. R., Ottusch, T. M., Jones, T., \& Richards, L. N. (2017). Understanding collegestudent roles: Perspectives of participants in a high school/community college dual-enrollment program. Community College Journal of Research and Practice, $1-17$.

Lindbeck, R., \& Fodrey, B. (2010). Using technology in undergraduate admission: A student perspective. Journal of College Admission, 208, 10-17.

Liu, P., \& Shi, J. (2018). Transmission of service from leader to followers: A parallel multiple mediator model. Social Behavior and Personality, 46(5), 769-782.

Lodge, J., \& Matthews, K. (2017). Evidence-based practice in higher education: Is it possible? Is it feasible? Paper presented at the Higher Education Research and Development Society of Australasia (HERDSA).

Lotkowski, V. A., Robbins, S. B., \& Noeth, R. J. (2004). The role of academic and nonacademic factors in improving college retention. . Retrieved from American College Testing ACT Inc:

Louis, K. S., Louis, K. S., Murphy, J., \& Murphy, J. (2017). Trust, caring and organizational learning: the leader's role. Journal of Educational Administration, 55(1), 103-126.

Loveridge, R., \& Pitt, M. (1990). The strategic management of technological innovation. Chichester, UK: Wiley

Lozano, R. (2014). Creativity and organizational learning as means to foster sustainability. Sustainability Development, 22(3), 205-216.

Magnusen, M. J., Kim, Y., Perrewé, P. L., \& Ferris, G. R. (2014). A critical review and synthesis of student-athlete college choice factors: Recruiting effectiveness in NCAA sports. International Journal of Sports Science \& Coaching, 9(6), 12651286.

Mahr, D., Lievens, A., \& Blazevic, V. (2014). The value of customer cocreated knowledge during the innovation process. Journal of Product Innovation Management, 31(3), 599-615.

Mandinach, E. B. (2012). A perfect time for data use: Using data-driven decision making to inform practice. Educational Psychologist, 47(2), 71-85.

Manski, C. F., \& Wise, D. A. (1983). College choice in America: Harvard University Press.

March, J. G. (1991). Exploration and exploitation in organizational learning. Organization Science, 2(1), 71-87. 
Márquez-Vera, C., Cano, A., Romero, C., Noaman, A. Y. M., Mousa Fardoun, H., \& Ventura, S. (2016). Early dropout prediction using data mining: a case study with high school students. Expert Systems, 33(1), 107-124.

Marsh, J. A., \& Farrell, C. C. (2015). How leaders can support teachers with data-driven decision making: A framework for understanding capacity building. Educational Management Administration \& Leadership, 43(2), 269-289.

Marsh, J. A., Pane, J. F., \& Hamilton, L. S. (2006). Making sense of data-driven decision making in education. Rand Education, OP-170-EDU, 1-16.

Mattern, K. D., Shaw, E. J., \& Kobrin, J. L. (2010). Academic fit: is the right school the best school or is the best school the right school? Journal of Advanced Academics, 21(3), 368-391.

Mena, J. A., \& Chabowski, B. R. (2015). The role of organizational learning in stakeholder marketing. Journal of the Academy of marketing science, 43, 429452.

Merriam, S. B., \& Tisdell, E. J. (2016). Qualitative research: A guide to design and implementation. San Francisco, CA: John Wiley \& Sons.

Miao, K. (2012). Performance-Based funding of higher education: A detailed look at best practices in 6 states. Center for American Progress, 1-12.

Miles, R. E., Snow, C. C., Meyer, A. D., \& Coleman Jr, H. (1978). Organizational strategy, structure, and process. Academy of Management Review, 3(3), 546-562.

Miller, N. B., \& Bell, B. (2016). Analytics to action: Predictive model outcomes and a communication strategy for student persistence. The Journal of Continuing Higher Education, 64(1), 16-29.

Moakler, M. W., \& Kim, M. M. (2014). College major choice in STEM: Revisiting confidence and demographic factors. The Career Development Quarterly, 62(2), 128-142.

Mora, V. J. (2003). Applications of GIS in admissions and targeting recruiting efforts. New Directions for Institutional Research, 2003(120), 15-21.

Mowery, D. C. (1981). The emergence and growth of industrial research in American manufacturing, 1899-1945. Stanford, CA: Stanford University.

Museus, S. D. (2014). The culturally engaging campus environments (CECE) model: A new theory of success among racially diverse college student populations. In Higher education: Handbook of theory and research (pp. 189-227). New York, NY: Springer. 
Natek, S., \& Zwilling, M. (2014). Student data mining solution-knowledge management system related to higher education institutions. Expert Systems with Applications, 41(14), 6400-6407.

NCLB. (2001). United States Department of Education. Public Law, 107-110.

Nichols, T. J., \& Chang, K.-L. (2013). Factors influencing honors college recruitment, persistence, and satisfaction at an upper-midwest land grant university. Journal of the National Collegiate Honors Council, 14(2), 105-127.

Nonaka, I., \& Takeuchi, H. (1995). The knowledge-creating company: How Japanese companies create the dynamics of innovation. New York, NY: Oxford University Press.

Northouse, P. G. (2016). Leadership Theory and Practice. Thousand Oaks, CA: Sage Publications, Inc.

Noruzy, A., Dalfard, V. M., Azhdari, B., Nazari-Shirkouhi, S., \& Rezazadeh, A. (2013). Relations between transformational leadership, organizational learning, knowledge management, organizational innovation, and organizational performance: an empirical investigation of manufacturing firms. The International Journal of Advanced Manufacturing Technology, 1-13.

Paulsen, M. B. (1990). College Choice: Understanding student enrollment behavior. ASHE-ERIC Higher Education Report No. 6. George Washington University School of Education and Human Development, Washington, DC: ERIC.

Paulsen, M. B., \& John, E. P. S. (2002). Social class and college costs: Examining the financial nexus between college choice and persistence. The Journal of Higher Education, 73(2), 189-236.

Payne, A., \& Frow, P. (2005). A strategic framework for customer relationship management. Journal of Marketing, 69(4), 167-176.

Permzadian, V., \& Credé, M. (2016). Do first-year seminars improve college grades and retention? A quantitative review of their overall effectiveness and an examination of moderators of effectiveness. Review of Educational Research, 86(1), 277-316.

Perna, L. W. (2000). Differences in the decision to attend college among African Americans, Hispanics, and Whites. The Journal of Higher Education, 71(2), 117141.

Perna, L. W. (2006). Studying college access and choice: A proposed conceptual model. In J. C. Smart (Ed.), Higher Education: Handbook of theory and research (Vol. XXI, pp. 99-157). New York: Springer Press. 
Picciano, A. G. (2012). The evolution of big data and learning analytics in american higher education. Journal of Asynchronous Learning Networks, 16(3), 9-20.

Ponnuswamy, I., \& Manohar, H. L. (2016). Impact of learning organization culture on performance in higher education institutions. Studies in Higher Education, 41(1), 21-36.

Poole, S. M., Levin, M. A., \& Elam, K. (2017). Getting out of the rankings game: a better way to evaluate higher education institutions for best fit. Journal for Marketing in Higher Education, 1-20. doi:10.1080/08841241.2017.1311981

Popham, W. J. (1987). The merits of measurement-driven instruction. The Phi Delta Kappan, 68(9), 679-682.

Popham, W. J., Cruse, K. L., Rankin, S. C., Sandifer, P. D., \& Williams, P. L. (1985). Measurement-driven instruction: It's on the road. The Phi Delta Kappan, 66(9), 628-634.

Price, L., \& Kirkwood, A. (2014). Using technology for teaching and learning in higher education: A critical review of the role of evidence in informing practice. Higher Education Research \& Development, 33(3), 549-564.

Provost, F., \& Fawcett, T. (2013). Data science and its relationship to big data and datadriven decision making. Big Data, 1(1), 51-59.

Rafferty, A. E., \& Griffin, M. A. (2004). Dimensions of transformational leadership: Conceptual and empirical extensions. The Leadership Quarterly, 15(3), 329-354.

Rahimnia, F., \& Sharifirad, M. S. (2015). Authentic leadership and employee well-being: The mediating role of attachment insecurity. Journal of Business Ethics, 132(2), 363-377.

Real, J. C., Roldán, J. L., \& Leal, A. (2014). From entrepreneurial orientation and learning orientation to business performance: analysing the mediating role of organizational learning and the moderating effects of organizational size. British Journal of Management, 25(2), 186-208.

Reese, C., \& Hunter, D. (2016). What about the middle man? The impact of middle level managers on organizational learning. Journal of Management, 4(1), 17-25.

Regents, T. B. o. (2018). Board of Regents. Retrieved from https://www.texastech.edu/board-of-regents/past-meetings.php

Research, C. f. P. (2017). Carnegie Classification of Institutions of Higher Education. Retrieved from http://carnegieclassifications.iu.edu/definitions.php 
Riley, T. (2018, March 24, 2018) /Interviewer: J. D. Gragg.

Romero, C., \& Ventura, S. (2013). Data mining in education. Wiley Interdisciplinary Reviews: Data Mining and Knowledge Discovery, 3(1), 12-27.

Romsa, K., Bremer, K. L., \& Lewis, J. (2017). The evolution of student-faculty interactions: What matters to millennial college students? College Student Affairs Journal, 35(2), 85-99.

Rutherford, A., \& Rabovsky, T. (2014). Evaluating impacts of performance funding policies on student outcomes in higher education. The ANNALS of the American Academy of Political and Social Science, 655(1), 185-208.

Rygielski, C., Wang, J.-C., \& Yen, D. C. (2002). Data mining techniques for customer relationship management. Technology in Society, 24(4), 483-502.

Sailesh, S. B., Lu, K. J., \& Al Aali, M. (2016). Context driven data mining to classify students of higher educational institutions. Adaptation Interaction, 21, 217-248.

Sarros, J. C., \& Santora, J. C. (2001). The transformational-transactional leadership model in practice. Leadership \& Organization Development Journal, 22(8), 383394.

Sav, T. G. (2016). Declining state funding and efficiency effects on public higher education: Government really does matter. International Atlantic Economic Society, 22, 397-408.

Schilling, M. A., \& Fang, C. (2014). When hubs forget, lie, and play favorites: Interpersonal network structure, information distortion, and organizational learning. Strategic Management Journal, 35(7), 974-994.

Seeman, E. D., \& O'Hara, M. (2006). Customer relationship management in higher education: Using information systems to improve the student-school relationship. Campus-Wide Information Systems, 23(1), 24-34.

Senge, P. M. (1990). The Fifth Discipline: The art and Practice of the Learning Organization. New York, NY: Doubleday Currency.

Sidelinger, R. J., Frisby, B. N., \& Heisler, J. (2016). Students' out of the classroom communication with instructors and campus services: Exploring social integration and academic involvement. Learning and Individual Differences, 47, 167-171.

Skinner, B. T. (2018). Choosing college in the 2000s: An updated analysis using the conditional logistic choice model. Research in Higher Education, 1-31. 
Slavin, R. E. (2002). Evidence-based education policies: Transforming educational practice and research. Educational Researcher, 31(7), 15-21.

Solorzano, D. G., \& Ornelas, A. (2004). A critical race analysis of Latina/o and African American advanced placement enrollment in public high schools. The High School Journal, 87(3), 15-26.

Song, C., Park, K., \& Kang, S.-W. (2015). Servant leadership and team performance: The mediating role of knowledge sharing climate. Social Behavior and Personality, 43(10), 1749-1760.

Stanton, A., Black, T., Dhaliwal, R., \& Hutchinson, C. (2017). Building partnerships to enhance student well-being and strategic enrollment management. Strategic Enrollment Management Quarterly, 4(4), 156-160.

Stewart, J., \& Heaney, A. (2013). Admission rhetoric and academic self-efficacy: The importance of first communications for conditionally admitted students. Journal of College Admission, 219, 26-31.

Strayhorn, T. L. (2014). What role does grit play in the academic success of black male collegians at predominantly white institutions? Journal of African American Studies, 18(1), 1-10.

Sutin, S. E. (2018). Reforming higher education from within: Lessons learned from other mature sectors of the economy. Global Trends in Higher Education Financing, International Journal of Educational Development, 58, 18-25.

Tapp, A., Hicks, K., \& Stone, M. (2004). Direct and database marketing and customer relationship management in recruiting students for higher education. International Journal of Nonprofit Voluntary Sector Marketing, 9(4), 335-345.

Tepper, B. J., Dimotakis, N., Lambert, L. S., Koopman, J., Matta, F. K., Man Park, H., \& Goo, W. (2018). Examining follower responses to transformational leadership from a dynamic, person-environment fit perspective. Academy of Management Journal, 61(4), 1343-1368.

Thammasiri, D., Delen, D., Meesad, P., \& Kasap, N. (2014). A critical assessment of imbalanced class distribution problem: The case of predicting freshmen student attrition. Expert Systems with Applications, 41(2), 321-330.

Thomas, E., Dawes, W., \& Reznik, G. (2001). Using predictive modeling to target student recruitment: Theory and practice. AIR Professional File, 78(11).

Thomas, G. (2015). How to do your case study. Thousand Oaks, CA: Sage. 
Tinto, V. (1975). Dropout from higher education: A theoretical synthesis of recent research. Review of Educational Research, 89-125.

Tinto, V. (1987). Leaving college: Rethinking the causes and cures of student attrition. Chicago, IL: University of Chicago Press.

Tinto, V. (2006). Research and practice of student retention: What next? Journal of College Student Retention: Research, Theory \& Practice, 8(1), 1-19.

Tsang, E. W. (1997). Organizational learning and the learning organization: a dichotomy between descriptive and prescriptive research. Human Relations, 50(1), 73-89.

Tschechtelin, J. D. (2011). Increased enrollment + student success - funding = ? New Directions for Community Colleges(156), 49-59.

TTU. (2018). Undergraduate Admissions Office. Retrieved from https://www.depts.ttu.edu/admissions/staff/departmental/index.php

Tucker, L., \& McKnight, O. (2017). Assessing the validity of college success indicators for the at-risk student: toward developing a best-practice model. Journal of College Student Retention: Research, Theory \& Practice. doi:1521025117696822

Turner, P., \& Thompson, E. (2014). College retention initiatives meeting the needs of millennial freshman students. College student journal, 48(1), 94-104.

VanderSchee, B. A. (2009). Embracing enrollment management: A comprehensive approach to college student marketing. Academy of Marketing Studies Journal, $13(1)$.

Vianden, J., \& Barlow, P. J. (2014). Showing the love: Predictors of student loyalty to undergraduate institutions. Journal of Student Affairs Research and Practice, 51(1), 16-29.

Voyer, D., \& Voyer, S. D. (2014). Gender differences in scholastic achievement: A metaanalysis. Psychological Bulletin, 140(4), 1174.

Webber, D. A. (2016). Are college costs worth it? How ability, major, and debt affect the returns to schooling. Economics of Education Review, 53, 296-310.

Weber, M. (1947). The theory of social and economic organization (T. Parsons, Trans.). New York, NY: Free Press.

Weiss, M., Razinskas, S., Backmann, J., \& Hoegl, M. (2018). Authentic leadership and leaders' mental well-being: An experience sampling study. The Leadership Quarterly, 29(2), 309-321. 
Wibrowski, C. R., Matthews, W. K., \& Kitsantas, A. (2017). The role of a skills learning support program on first-generation college students' self-regulation, motivation, and academic achievement: A longitudinal study. Journal of College Student Retention: Research, Theory, Practice, 19(3), 317-332.

WICHE. (2019). Retrieved from https://knocking.wiche.edu/

Williams, J. (2016). A critical exploration of changing definitions of public good in relation to higher education. Studies in Higher Education, 41(4), 619-630.

Witteveen, D., \& Attewell, P. (2017). The college completion puzzle: A hidden markov model approach. Research in Higher Education, 58(4), 449-467.

Young, J. W., \& Johnson, P. M. (2004). The impact of an SES-based model on a college's undergraduate admissions outcomes. Research in Higher Education, 45(7), 777-797. 
SECTION SIX

SCHOLARLY PRACTICE REFLECTION 
As I reflect on my time in this program, I think of both the relationships I have gained as well as the knowledge. I planned on gaining knowledge since that was the whole point of starting a doctoral program, but the networking and friends were a bonus. I began the program excited to learn, especially more about leadership, as that is my passion and what my Masters degree was based. I appreciated the on-site component; however, it was that part I was most hesitant about in the beginning. I initially planned to keep to myself, knowing that I did not enjoy group projects from experience. Initially, that was what I did until the wicked problem forced me to branch out and take an active role. I gained so much from the high quality group work and witness every style and frame of leadership we were studying in those short four weeks of summer one. Upon meeting and joining my group for the fall and spring semesters, I found friends and colleagues for life. I never would have expected to find a best friend, especially one so different from me. I learned at least as much from the group interactions and my colleagues as I did from all of the reading and writing. Both were valuable, but one was expected, and one was not. It seems to me, upon reflection, that the unexpected outcome will leave a larger impact on my life.

As a result, this program has made me a better leader. The one thing I learned and saw in practice is that "one size fits all leadership" does not work. I knew that in theory and felt like I did a good job of implementing that into my daily life. However, through the group work throughout the program, I learned a new level of compromise, team work, and how, within a single project, leadership can switch amongst participants multiple times, and that is okay and can be beneficial. Additionally, I hope that I have became a better writer and I know I became a better researcher. I use data daily in my current job as 
a data analyst. I would have never thought I could be happy with that as a career, but the research for this dissertation and the resulting analysis showed that I truly enjoy the data aspects of enrollment management.

From my research, I was happy to learn that data, culture, art, and science, along with numbers and feelings, are important in the recruitment of students. Data was becoming more and more important, but equally important is the human aspect that must not be ignored.

My final reflection is that while I initially thought this dissertation was more of a hoop to jump through, I found value in it beyond just the accomplishment of completing it. I think I discovered many ideas and practices that can be useful to colleges and universities and help in informing best practices. This is highly rewarding for three plus years of work. I am thankful for my time in this program and feel like I have grown as a professional, which matters a great deal. In deciding whether to take on this goal of completing a doctoral program, I talked to far too many people who said they felt like their journey did not matter, they just wanted and received the piece of paper at the end. I feel like I did learn, I know I grew, and I am better for completing this degree. 


\section{References}

Adelman, C. (2006). The toolbox revisited: Paths to degree completion from high school through college. Washington, DC.

Agarwal, R., \& Helfat, C. E. (2009). Strategic renewal of organizations. Organization Science, 20(2), 281-293.

An, B. P., \& Sorensen, K. N. (2017). Family stucture changes during high school and college selectivity. Research in Higher Education, 1-28.

Antons, C. M., \& Maltz, E. N. (2006). Expanding the role of institutional research at small private universities: A case study in enrollment management using data mining. New Directions for Institutional Research, 2006(131), 69-81.

Argote, L., \& Miron-Spektor, E. (2011). Organizational learning: From experience to knowledge. Organization science, 22(5), 1123-1137.

Attaran, M., Stark, J., \& Stotler, D. (2018). Opportunities and challenges for big data analytics in US higher education: A conceptual model for implementation. Industry and Higher Education, 32(3), 169-182.

Attewell, P. (1992). Technology diffusion and organizational learning: The case of business computing. Organization Science, 3(1), 1-19.

Baden-Fuller, C., \& Volberda, H. W. (1997). Strategic renewal: How large complex organizations prepare for the future. International Studies of Management \& Organization, 27(2), 95-120.

Baker, V. L., \& Baldwin, R. G. (2015). A case study of liberal arts colleges in the $21 \mathrm{st}$ century: Understanding organizational change and evolution in higher education. Innovative Higher Education, 40(3), 247-261.

Barton, A. L., \& Hirsch, J. K. (2016). Permissive parenting and mental health in college students: Mediating effects of academic entitlement. 64(1), 1-8.

Baum, S., Kurose, C., \& McPherson, M. (2013). An overview of American higher education. The Future of Children, 23(1), 17-39.

Bean, J., \& Hossler, D. (1990). The strategic management of college enrollments. San Franciso, CA: Jossey-Bass.

Bell, B. J., Gass, M. A., Nafziger, C. S., \& Starbuck, J. D. (2014). The state of knowledge of outdoor orientation programs: Current practices, research, and theory. 37(1), $31-45$. 
Berry, L. L. (1995). Relationship marketing of services—growing interest, emerging perspectives. Journal of the Academy of marketing science, 23(4), 236-245.

Bertaux, D. (1981). Bibliography and society: The life history approach in the social sciences. Thousand Oaks, CA: Sage.

Biesta, G. (2007). Why "what works" won't work: Evidence-based practice and the democratic deficit in educational research. Educational Theory, 57(1), 1-22.

Bolman, L. G., \& Deal, T. E. (2013). Reframing organizations: Artistry, choice, and leadership. San Francisco, CA: John Wiley \& Sons.

Bontrager, B. (2004). Strategic enrollment management: Core strategies and best practices. College and University, 79(4), 9.

Bouwma-Gearhart, J., \& Collins, J. (2015). What we know about data-driven decision making in higher education: Informing educational policy and practice. Paper presented at the Proceedings of International Academic Conferences.

Bowen, W. G., Bok, D., \& Burkhart, G. (1998). A report card on diversity: lessons for business from higher education. Harvard Business Review, 77(1), 138-149.

Brotherton, P. (2001). It takes a campus to graduate a student: A look at seven academic retention programs and what makes them effective. Diverse Issues in Higher Education, 18(18), 34.

Brown, M. G., Wohn, D. Y., \& Ellison, N. (2016). Without a map: College access and the online practices of youth from low-income communities. Computers \& Education, 92, 104-116.

Cabrera, A. F., Nora, A., \& Castaneda, M. B. (1993). College persistence: Structural equations modeling test of an integrated model of student retention. The Journal of Higher Education, 64(2), 123-139.

Calderon, A., \& Mathies, C. (2013). Institutional research in the future: Challenges within higher education and the need for excellence in professional practice. New Directions for Institutional Research, 2013(157), 77-90.

Cangelosi, V. E., \& Dill, W. R. (1965). Organizational learning: Observations toward a theory. Administrative Sciences Quarterly, 175-203.

Cegler, T. D. (2012). Targeted recruitment of GLBT students by colleges and universities. Journal of College Admission, 215, 18-23.

Chapman, R. (1984). Toward a theory of college choice: A model of college search and choice behavior. Alberta, Canada: University of Alberta. 
Chapman, R. L., Kennedy, J. L., Newell, A., \& Biel, W. C. (1959). The systems research laboratory's air defense experiments. Management Science, 5(3), 250-269.

Chart, T. O. (2018). Texas Tech University Organizational Chart. Retrieved from http://www.ttu.edu/administration/president/org-chart_10-3.pdf

Chiu, J., Economos, J., Markson, C., Raicovi, V., Howell, C., Morote, E.-S., \& Inserra, A. (2016). Which matters most? Perceptions of family income or parental education on academic achievement. New York Journal of Student Affairs, 16(2), 3-16.

Chiva, R. (2017). The learning organization and the level of consciousness. The Learning Organization, 24(3).

Choo, C. W. (1996). The knowing organization: How organizations use information to construct meaning, create knowledge and make decisionis. International Journal of Information Management, 16(5), 329-340.

Cook, S. D., \& Yanow, D. (1993). Culture and organizational learning. Journal of Management Inquiry, 2(4), 373-390.

Coopey, J. (1995). The learning organization, power, politics and ideology introduction. Management \& Learning, 26(2), 193-213.

Cox, B. E., Reason, R. D., Tobolowsky, B. F., Brower, R. L., Patterson, S., Luczyk, S., \& Roberts, K. (2017). Lip service or actionable insights? Linking student experiences to institutional assesment and data-driven decision making in higher education. The Journal of Higher Education, 88(6), 835-862.

Creswell, J. W. (2014). Research Design: Qualitative, quantitative, and mixed method approaches. Thousand Oaks, CA: Sage Publications.

Crossan, M. M., \& Berdrow, I. (2003). Organizational learning and strategic renewal. Strategic Management Journal, 24(11), 1087-1105.

Crossan, M. M., Lane, H. W., \& White, R. E. (1999). An organizational learning framework: From intuition to institution. Academy of Management Review, 24(3), 522-537.

Cunningham, A. (2014, March 12, 2014). Tech programs ranked in Top 100 in United States. The Daily Toreador.

Cyert, R. M., \& March, J. G. (1963). A behavioral theory of the firm. Englewood Cliffs, NJ: Prentice-Hall. 
Daniel, B. (2015). Big data and analytics in higher education: Opportunities and challenges. British Journal of Educational Technology, 46(5), 904-920.

Davidson, C., \& Wilson, K. (2013). Reassessing Tinto's concepts of social and academic integration in student retention. Journal of College Student Retention: Research, Theory \& Practice, 15(3), 329-346.

DeShields Jr, O. W., Kara, A., \& Kaynak, E. (2005). Determinants of business student satisfaction and retention in higher education: applying Herzberg's two-factor theory. International journal of educational management, 19(2), 128-139.

DesJardins, S. L. (2002). An analytic strategy to assist institutional recruitment and marketing efforts. Research in Higher Education, 43(5), 531-553.

DesJardins, S. L., Ahlburg, D. A., \& McCall, B. P. (2006). An integrated model of application, admission, enrollment, and financial aid. The Journal of Higher Education, 77(3), 381-429.

DesJardins, S. L., Dundar, H., \& Hendel, D. D. (1999). Modeling the college application decision process in a land-grant university. Economics of Education Review, 18(1), 117-132.

Dodgson, M. (1991). Technology learning, technology strategy and competitive pressures. British Journal of Management, 2(3), 133-149.

Dolence, M. G. (1998). Strategic enrollment management. Hand-book for the College Admissions Profession, 71-91.

Drazin, R., Glynn, M. A., \& Kazanijan, R. K. (1999). Multilevel theorizing about creativity in organizations: A sensemaking perspective. Academy of Management Review, 24(2), 286-307.

Duncan, R. B. (1974). Modifications in decision structure in adapting to the environment: Some implications for organizational learning. Decision Sciences, 5(4), 705-725.

Dutta, D. K., \& Crossan, M. M. (2005). The nature of entrepreneurial opportunities: Understanding the process using the 4I organizational learning framework. Entrepreneurship Theory and Practice, 29(4), 425-449.

Education, D. o. (2008). Higher Education Act. Retrieved from https://www.hacu.net/hacu/Higher_Education_Act_(HEA).asp

Ehrenberg, R. G., Zhang, L., \& Levin, J. (2005). Crafting a class: The trade off between merit scholarships and enrolling lower-income students. The Review of Higher Education, 29(2), 195-212. 
Elliott, K. M., \& Healy, M. A. (2001). Key factors influencing student satisfaction related to recruitment and retention. Journal for Marketing for Higher Education, 10(4), $1-11$.

Erdem, M., \& Ucar, I. H. (2013). Learning organization perceptions in elementary education in terms of teachers and the effect of learning organization on organizational commitment. Educational Sciences: Theory and Practice, 13(3), 1527-1534.

Eskreis-Winkler, L., Shulman, E. P., Beal, S. A., \& Duckworth, A. L. (2014). The grit effect: Predicting retention in the military, the workplace, school and marriage. Frontiers in Psychology, 5.

Facts, T. Q. (2018). Quick Facts. Retrieved from http://www.ttu.edu/about/

Ferris, E., Finster, M., \& McDonald, D. (2004). Academic fit of student-athletes: An analysis of NCAA division IA graduation rates. Research in Higher Education, $45(6), 555-575$.

Foote, S. M., Kranzow, J., \& Hinkle, S. E. (2015). Focusing on the forgotten: An examination of the influences and innovative practices that affect community college transfer student success. In Examining the Impact of Community Colleges on the Global Workforce (pp. 94-124): IGI Global.

Ford, W. G. (2011). Evaluating the effectiveness of college web sites for prospective students. Journal of College Admission, 212, 26-31.

Francis, D. E. (2014). Lean and the learning organization in higher education. Canadian Journal of Educational Administration and Policy, 157, 1-23.

Freeman, K. (1997). Increasing African Americans' participation in higher education: African American high-school students' perspectives. Journal of Higher education, 68(5), 523-550.

Freitas, S. I., Morgan, J., \& Gibson, D. (2015). Will MOOCs transform learning and teaching in higher education? Engagement and course retention in online learning provision. British Journal of Educational Technology, 46(3), 455-471.

Fuller, B., Wright, J., Gesicki, K., \& Kang, E. (2007). Gauging growth: How to judge no child left behind? Educational Researcher, 36(5), 268-278.

Gikas, J., \& Grant, M. M. (2013). Mobile computing devices in higher education: Student perspectives on learning with cellphones, smartphones \& social media. The Internet and Higher Education, 19, 18-26. 
Goenner, C. F., \& Pauls, K. (2006). A predictive model of inquiry to enrollment. Research in Higher Education, 47(8), 935-956.

Goldstein, P. J., \& Katz, R. N. (2005). Academic Analytics: The uses of management information and technology in higher education. Washington, D.C.: Educause Center for Applied Research.

Greene, J. P., \& Forster, G. (2003). Public high school graduation and college readiness rates in the United States. Education Working Paper No. 3. Center for Civic Innovation.

Greenleaf, R. K. (1970). The Servant as Leader. The Robert K. Greenleaf Center, Indianapolis, IN.

Guinot, J., Chiva, R., \& Mallén, F. (2016). Linking altruism and organizational learning capability: A study from excellent human resources management organizations in Spain. Journal of Business Ethics, 138(2), 349-364.

Gulick, L., \& Urwick, L. (Eds.). (1937). Papers on the science of administration. New York, NY: Columbia University Press.

HACU. (2017). Hispanic Serving Institutions Defintions. Retrieved from https://www.hacu.net/hacu/HSI_Definition1.asp

Han, P. (2014). A literature review on college choice and marketing strategies for recruitment. Family and Consumer Sciences Research Journal, 43(2), 120-130.

Hancock, D. R., \& Algozzine, B. (2015). Doing case study research: A practical guide for beginning researchers. New York, NY: Teachers College Press.

Hanushek, E., \& Lindseth, A. (2009). Performance-based funding. Education Week, 28, 28.

Hill, C. B., \& Winston, G. C. (2010). Low-income students and highly selective private colleges: Geography, searching, and recruiting. Economics of Education Review, 29(4), 495-503.

Hoppes, C. R., \& Holley, K. A. (2014). Organizational trust in times of challenge: The impact on faculty and administrators. Innovative Higher Education, 39(3), 201216.

Hora, M. T., Bouwma-Gearhart, J., \& Park, H. J. (2017). Data driven decision-making in the era of accountability: Fostering faculty data cultures for learning. The Review of Higher Education, 40(3), 391-426. 
Hossler, D., \& Gallagher, K. S. (1987). Studying student college choice: A three-phase model and the implications for policymakers. College and University, 62(3), 207221.

Hossler, D., \& Kalsbeek, D. (2013). Enrollment management and managing enrollments: Revisiting the context for institutional strategy. Strategic Enrollment Management Quarterly, 1(1), 5-25.

Hotho, J. J., Lyles, M. A., \& Easterby-Smith, M. (2015). The mutual impact of global strategy and organizational themes and future directions. Global Strategy Journal, $5,85-112$.

Hotho, J. J., Saka-Helmhout, A., \& Becker-Ritterspach, F. (2014). Bringing context and structure back into situated learning. Management learning, 45(1), 57-80.

Hubbard, L., Datnow, A., \& Pruyn, L. (2014). Multiple initiatives, multiple challenges: The promise and pitfalls of implementing data. Studies in Educational Evaluation, $42,54-62$.

Hult, G. T. M., Mena, J. A., Ferrell, O. C., \& Ferrell, L. (2011). Stakeholder marketing: A definition and conceptual framework. AMS Review, 1(1), 44-65.

Hurst, D. K. (1995). Crisis \& renewal: Harvard Business School Press Boston, MA.

Hussar, W. J., \& Bailey, T. M. (2016). Projections of education statistics to 2023. NCES 2015-073. National Center for Education Statistics, 42, 1-157.

Hwang, Y.-S., \& Choi, Y. K. (2019). Higher education service quality and student satisfaction, institutional image, and behavioral intention. Social Behavior and Personality: An International Journal, 47(2), 1-12.

IIT. (2018). Quick Facts. Retrieved from https://web.iit.edu/about/quick-facts

IPEDS. IES/NCES National Center for Education Statistics. Retrieved from https://nces.ed.gov/ipeds/datacenter/

Irlbeck, E., Adams, S., Akers, C., Burris, S., \& Jones, S. (2014). First generation college students: Motivations and support systems. Journal for Agricultural Education, 55(2), 154-166.

Isaacs, M. L. (2003). Data-driven decision making: The engine of accountability. Professional School Counseling, 6(4), 288-295.

Jackson, G. A. (1982). Public efficiency and private choice in higher education. Educational Evaluation and Policy Analysis, 4(2), 237-247. 
Jelinek, M. (1979). Institutionalizing innovation: A study of organizational learning systems: Greenwood.

John, E. P. S. (1990). Price response in enrollment decisions: An analysis of the high school and beyond sophomore cohort. Research in Higher Education, 31(2), 161176.

Jose, M., Kurian, P. S., \& Biju, V. (2016). Progression analysis of students in a higher education institution using big data open source predictive modeling tool. Paper presented at the Big Data and Smart City (ICBDSC), 2016 3rd MEC International Conference.

Jyoti, J., \& Bhau, S. (2016). Transformational leadership and job performance: A study of higher education. Journal of Services Research, 15(2), 77-110.

Kabakchieva, D. (2013). Predicting student performance by using data mining methods for classification. Cybernetics \& Information Technologies, 13(1), 61-72.

Kaemar, M., Harris, K., Andrews, M., \& Tepper, B. J. (2013). Ethical leadership and subordinate outcomes: The mediating role of organizational politics and the moderating role of political skill. Journal of Business Ethics, 115, 33-44.

Kansal, A. K., \& Kaur, H. (2016). A study of career orientation of female college students in relation to parental education and employment status. International Education and Research Journal, 2(9).

Kao, G., \& Thompson, J. S. (2003). Racial and ethnic stratification in educational achievement and attainment. Annual review of sociology, 29(1), 417-442.

Kaufman, T. E., Graham, C. R., Picciano, A. G., Popham, J. A., \& Wiley, D. (2014). Data-driven decision making in the K-12 classroom. In Handbook of Research on Educational Communications \& Technology (pp. 337-346). New York, NY: Springer Press.

Kemerer, F. R., Baldridge, J. V., \& Green, K. C. (1982). Strategies for effective enrollment management: Amer Assn of State Colleges \&.

Kowalski, T. (2010). Handbook of Data-Based Decision Making in Education. New York, NY: Routledge Press.

Kretchmar, J., \& Memory, A. (2010). One college's journey into the unconscious mind of its prospective students: How a new research methodology is helping us recruit. Journal of College Admission, 207, 8-15.

Krueger, R. A., \& Casey, M. A. (2015). Focus groups: A practical guide for applied research: Sage publications. 
Kuh, G. D., Kinzie, J., Schuh, J. H., \& Whitt, E. J. (2011). Student success in college: Creating conditions that matter. San Franciso, CA: John Wiley \& Sons.

Lambert, L. S., Tepper, B. J., Carr, J. C., Holt, D. T., \& Barelka, A. J. (2012). Forgotten but not gone: An examination of fit between leader consideration and initiating structure needed and received. Journal of Applied Psychology, 97(5), 913.

Langley, A., Smallman, C., Tsoukas, H., \& Van de Ven, A. H. (2013). Process studies of change in organization and management: Unveiling temporality, activity, and flow. Academy of Management Journal, 56(1), 1-13.

Langston, R., \& Loreto, D. (2017). Seamless integration of predictive analytics and CRM within an undergraduate admissions recruitment and marketing plan. Strategic Enrollment Management Quarterly, 4(4), 161-172.

Lawrence, T. B., Mauws, M. K., Dyck, B., \& Kleysen, R. F. (2005). The politics of organizational learning: Integrating power into the 4I framework. Academy of Management Review, 30(1), 180-191.

Le, H., Robbins, S. B., \& Westrick, P. (2014). Predicting student enrollment and persistence in college STEM fields using an expanded PE fit framework: A largescale multilevel study. Journal of Applied Psychology, 99(5), 915.

Leroy, H., Anseel, F., Gardner, W. L., \& Sels, L. (2015). Authentic leadership, authentic followership, basic need satisfaction, and work role performance: A cross-level study. Journal of Management, 41(6), 1677-1697.

Levant, R., \& Silverstein, L. (2005). Gender is neglected in both evidence-based practices and "treatment as usual". Washington D.C.: APA Books.

Levi, D. (2014). Group dynamics for teams. New York, NY: Sage Publications.

Levin, R. (2015). Strategic Alignment: Recruiting students in a highly decentralized environment. College and University, 91(1), 47-54.

Levine, A., \& Dean, D. R. (2013). It's only technology if it happens after you are born. Journal of College Admission, 220, 6-12.

Levitt, B., \& March, J. G. (1988). Organizational learning. Annual Review for Sociology, 14(1), 319-338.

Lew, M. M., \& Nelson, R. F. (2016). New teachers' challenges: how culturally responsive teaching, classroom management, \& assessment literacy are intertwined. Multicultural Education, 23(3/4), 7. 
Lile, J. R., Ottusch, T. M., Jones, T., \& Richards, L. N. (2017). Understanding collegestudent roles: Perspectives of participants in a high school/community college dual-enrollment program. Community College Journal of Research and Practice, $1-17$.

Lindbeck, R., \& Fodrey, B. (2010). Using technology in undergraduate admission: A student perspective. Journal of College Admission, 208, 10-17.

Liu, P., \& Shi, J. (2018). Transmission of service from leader to followers: A parallel multiple mediator model. Social Behavior and Personality, 46(5), 769-782.

Lodge, J., \& Matthews, K. (2017). Evidence-based practice in higher education: Is it possible? Is it feasible? Paper presented at the Higher Education Research and Development Society of Australasia (HERDSA).

Lotkowski, V. A., Robbins, S. B., \& Noeth, R. J. (2004). The role of academic and nonacademic factors in improving college retention. . Retrieved from American College Testing ACT Inc:

Louis, K. S., Louis, K. S., Murphy, J., \& Murphy, J. (2017). Trust, caring and organizational learning: the leader's role. Journal of Educational Administration, 55(1), 103-126.

Loveridge, R., \& Pitt, M. (1990). The strategic management of technological innovation. Chichester, UK: Wiley

Lozano, R. (2014). Creativity and organizational learning as means to foster sustainability. Sustainability Development, 22(3), 205-216.

Magnusen, M. J., Kim, Y., Perrewé, P. L., \& Ferris, G. R. (2014). A critical review and synthesis of student-athlete college choice factors: Recruiting effectiveness in NCAA sports. International Journal of Sports Science \& Coaching, 9(6), 12651286.

Mahr, D., Lievens, A., \& Blazevic, V. (2014). The value of customer cocreated knowledge during the innovation process. Journal of Product Innovation Management, 31(3), 599-615.

Mandinach, E. B. (2012). A perfect time for data use: Using data-driven decision making to inform practice. Educational Psychologist, 47(2), 71-85.

Manski, C. F., \& Wise, D. A. (1983). College choice in America: Harvard University Press.

March, J. G. (1991). Exploration and exploitation in organizational learning. Organization Science, 2(1), 71-87. 
Márquez-Vera, C., Cano, A., Romero, C., Noaman, A. Y. M., Mousa Fardoun, H., \& Ventura, S. (2016). Early dropout prediction using data mining: a case study with high school students. Expert Systems, 33(1), 107-124.

Marsh, J. A., \& Farrell, C. C. (2015). How leaders can support teachers with data-driven decision making: A framework for understanding capacity building. Educational Management Administration \& Leadership, 43(2), 269-289.

Marsh, J. A., Pane, J. F., \& Hamilton, L. S. (2006). Making sense of data-driven decision making in education. Rand Education, OP-170-EDU, 1-16.

Mattern, K. D., Shaw, E. J., \& Kobrin, J. L. (2010). Academic fit: is the right school the best school or is the best school the right school? Journal of Advanced Academics, 21(3), 368-391.

Mena, J. A., \& Chabowski, B. R. (2015). The role of organizational learning in stakeholder marketing. Journal of the Academy of marketing science, 43, 429452.

Merriam, S. B., \& Tisdell, E. J. (2016). Qualitative research: A guide to design and implementation. San Francisco, CA: John Wiley \& Sons.

Miao, K. (2012). Performance-Based funding of higher education: A detailed look at best practices in 6 states. Center for American Progress, 1-12.

Miles, R. E., Snow, C. C., Meyer, A. D., \& Coleman Jr, H. (1978). Organizational strategy, structure, and process. Academy of Management Review, 3(3), 546-562.

Miller, N. B., \& Bell, B. (2016). Analytics to action: Predictive model outcomes and a communication strategy for student persistence. The Journal of Continuing Higher Education, 64(1), 16-29.

Moakler, M. W., \& Kim, M. M. (2014). College major choice in STEM: Revisiting confidence and demographic factors. The Career Development Quarterly, 62(2), $128-142$.

Mora, V. J. (2003). Applications of GIS in admissions and targeting recruiting efforts. New Directions for Institutional Research, 2003(120), 15-21.

Mowery, D. C. (1981). The emergence and growth of industrial research in American manufacturing, 1899-1945. Stanford, CA: Stanford University.

Museus, S. D. (2014). The culturally engaging campus environments (CECE) model: A new theory of success among racially diverse college student populations. In Higher education: Handbook of theory and research (pp. 189-227). New York, NY: Springer. 
Natek, S., \& Zwilling, M. (2014). Student data mining solution-knowledge management system related to higher education institutions. Expert Systems with Applications, 41(14), 6400-6407.

NCLB. (2001). United States Department of Education. Public Law, 107-110.

Nichols, T. J., \& Chang, K.-L. (2013). Factors influencing honors college recruitment, persistence, and satisfaction at an upper-midwest land grant university. Journal of the National Collegiate Honors Council, 14(2), 105-127.

Nonaka, I., \& Takeuchi, H. (1995). The knowledge-creating company: How Japanese companies create the dynamics of innovation. New York, NY: Oxford University Press.

Northouse, P. G. (2016). Leadership Theory and Practice. Thousand Oaks, CA: Sage Publications, Inc.

Noruzy, A., Dalfard, V. M., Azhdari, B., Nazari-Shirkouhi, S., \& Rezazadeh, A. (2013). Relations between transformational leadership, organizational learning, knowledge management, organizational innovation, and organizational performance: an empirical investigation of manufacturing firms. The International Journal of Advanced Manufacturing Technology, 1-13.

Paulsen, M. B. (1990). College Choice: Understanding student enrollment behavior. ASHE-ERIC Higher Education Report No. 6. George Washington University School of Education and Human Development, Washington, DC: ERIC.

Paulsen, M. B., \& John, E. P. S. (2002). Social class and college costs: Examining the financial nexus between college choice and persistence. The Journal of Higher Education, 73(2), 189-236.

Payne, A., \& Frow, P. (2005). A strategic framework for customer relationship management. Journal of Marketing, 69(4), 167-176.

Permzadian, V., \& Credé, M. (2016). Do first-year seminars improve college grades and retention? A quantitative review of their overall effectiveness and an examination of moderators of effectiveness. Review of Educational Research, 86(1), 277-316.

Perna, L. W. (2000). Differences in the decision to attend college among African Americans, Hispanics, and Whites. The Journal of Higher Education, 71(2), 117141.

Perna, L. W. (2006). Studying college access and choice: A proposed conceptual model. In J. C. Smart (Ed.), Higher Education: Handbook of theory and research (Vol. XXI, pp. 99-157). New York: Springer Press. 
Picciano, A. G. (2012). The evolution of big data and learning analytics in american higher education. Journal of Asynchronous Learning Networks, 16(3), 9-20.

Ponnuswamy, I., \& Manohar, H. L. (2016). Impact of learning organization culture on performance in higher education institutions. Studies in Higher Education, 41(1), 21-36.

Poole, S. M., Levin, M. A., \& Elam, K. (2017). Getting out of the rankings game: a better way to evaluate higher education institutions for best fit. Journal for Marketing in Higher Education, 1-20. doi:10.1080/08841241.2017.1311981

Popham, W. J. (1987). The merits of measurement-driven instruction. The Phi Delta Kappan, 68(9), 679-682.

Popham, W. J., Cruse, K. L., Rankin, S. C., Sandifer, P. D., \& Williams, P. L. (1985). Measurement-driven instruction: It's on the road. The Phi Delta Kappan, 66(9), 628-634.

Price, L., \& Kirkwood, A. (2014). Using technology for teaching and learning in higher education: A critical review of the role of evidence in informing practice. Higher Education Research \& Development, 33(3), 549-564.

Provost, F., \& Fawcett, T. (2013). Data science and its relationship to big data and datadriven decision making. Big Data, 1(1), 51-59.

Rafferty, A. E., \& Griffin, M. A. (2004). Dimensions of transformational leadership: Conceptual and empirical extensions. The Leadership Quarterly, 15(3), 329-354.

Rahimnia, F., \& Sharifirad, M. S. (2015). Authentic leadership and employee well-being: The mediating role of attachment insecurity. Journal of Business Ethics, 132(2), 363-377.

Real, J. C., Roldán, J. L., \& Leal, A. (2014). From entrepreneurial orientation and learning orientation to business performance: analysing the mediating role of organizational learning and the moderating effects of organizational size. British Journal of Management, 25(2), 186-208.

Reese, C., \& Hunter, D. (2016). What about the middle man? The impact of middle level managers on organizational learning. Journal of Management, 4(1), 17-25.

Regents, T. B. o. (2018). Board of Regents. Retrieved from https://www.texastech.edu/board-of-regents/past-meetings.php

Research, C. f. P. (2017). Carnegie Classification of Institutions of Higher Education. Retrieved from http://carnegieclassifications.iu.edu/definitions.php 
Riley, T. (2018, March 24, 2018) /Interviewer: J. D. Gragg.

Romero, C., \& Ventura, S. (2013). Data mining in education. Wiley Interdisciplinary Reviews: Data Mining and Knowledge Discovery, 3(1), 12-27.

Romsa, K., Bremer, K. L., \& Lewis, J. (2017). The evolution of student-faculty interactions: What matters to millennial college students? College Student Affairs Journal, 35(2), 85-99.

Rutherford, A., \& Rabovsky, T. (2014). Evaluating impacts of performance funding policies on student outcomes in higher education. The ANNALS of the American Academy of Political and Social Science, 655(1), 185-208.

Rygielski, C., Wang, J.-C., \& Yen, D. C. (2002). Data mining techniques for customer relationship management. Technology in Society, 24(4), 483-502.

Sailesh, S. B., Lu, K. J., \& Al Aali, M. (2016). Context driven data mining to classify students of higher educational institutions. Adaptation Interaction, 21, 217-248.

Sarros, J. C., \& Santora, J. C. (2001). The transformational-transactional leadership model in practice. Leadership \& Organization Development Journal, 22(8), 383394.

Sav, T. G. (2016). Declining state funding and efficiency effects on public higher education: Government really does matter. International Atlantic Economic Society, 22, 397-408.

Schilling, M. A., \& Fang, C. (2014). When hubs forget, lie, and play favorites: Interpersonal network structure, information distortion, and organizational learning. Strategic Management Journal, 35(7), 974-994.

Seeman, E. D., \& O'Hara, M. (2006). Customer relationship management in higher education: Using information systems to improve the student-school relationship. Campus-Wide Information Systems, 23(1), 24-34.

Senge, P. M. (1990). The Fifth Discipline: The art and Practice of the Learning Organization. New York, NY: Doubleday Currency.

Sidelinger, R. J., Frisby, B. N., \& Heisler, J. (2016). Students' out of the classroom communication with instructors and campus services: Exploring social integration and academic involvement. Learning and Individual Differences, 47, 167-171.

Skinner, B. T. (2018). Choosing college in the 2000s: An updated analysis using the conditional logistic choice model. Research in Higher Education, 1-31. 
Slavin, R. E. (2002). Evidence-based education policies: Transforming educational practice and research. Educational Researcher, 31(7), 15-21.

Solorzano, D. G., \& Ornelas, A. (2004). A critical race analysis of Latina/o and African American advanced placement enrollment in public high schools. The High School Journal, 87(3), 15-26.

Song, C., Park, K., \& Kang, S.-W. (2015). Servant leadership and team performance: The mediating role of knowledge sharing climate. Social Behavior and Personality, 43(10), 1749-1760.

Stanton, A., Black, T., Dhaliwal, R., \& Hutchinson, C. (2017). Building partnerships to enhance student well-being and strategic enrollment management. Strategic Enrollment Management Quarterly, 4(4), 156-160.

Stewart, J., \& Heaney, A. (2013). Admission rhetoric and academic self-efficacy: The importance of first communications for conditionally admitted students. Journal of College Admission, 219, 26-31.

Strayhorn, T. L. (2014). What role does grit play in the academic success of black male collegians at predominantly white institutions? Journal of African American Studies, 18(1), 1-10.

Sutin, S. E. (2018). Reforming higher education from within: Lessons learned from other mature sectors of the economy. Global Trends in Higher Education Financing, International Journal of Educational Development, 58, 18-25.

Tapp, A., Hicks, K., \& Stone, M. (2004). Direct and database marketing and customer relationship management in recruiting students for higher education. International Journal of Nonprofit Voluntary Sector Marketing, 9(4), 335-345.

Tepper, B. J., Dimotakis, N., Lambert, L. S., Koopman, J., Matta, F. K., Man Park, H., \& Goo, W. (2018). Examining follower responses to transformational leadership from a dynamic, person-environment fit perspective. Academy of Management Journal, 61(4), 1343-1368.

Thammasiri, D., Delen, D., Meesad, P., \& Kasap, N. (2014). A critical assessment of imbalanced class distribution problem: The case of predicting freshmen student attrition. Expert Systems with Applications, 41(2), 321-330.

Thomas, E., Dawes, W., \& Reznik, G. (2001). Using predictive modeling to target student recruitment: Theory and practice. AIR Professional File, 78(11).

Thomas, G. (2015). How to do your case study. Thousand Oaks, CA: Sage. 
Tinto, V. (1975). Dropout from higher education: A theoretical synthesis of recent research. Review of Educational Research, 89-125.

Tinto, V. (1987). Leaving college: Rethinking the causes and cures of student attrition. Chicago, IL: University of Chicago Press.

Tinto, V. (2006). Research and practice of student retention: What next? Journal of College Student Retention: Research, Theory \& Practice, 8(1), 1-19.

Tsang, E. W. (1997). Organizational learning and the learning organization: a dichotomy between descriptive and prescriptive research. Human Relations, 50(1), 73-89.

Tschechtelin, J. D. (2011). Increased enrollment + student success - funding = ? New Directions for Community Colleges(156), 49-59.

TTU. (2018). Undergraduate Admissions Office. Retrieved from https://www.depts.ttu.edu/admissions/staff/departmental/index.php

Tucker, L., \& McKnight, O. (2017). Assessing the validity of college success indicators for the at-risk student: toward developing a best-practice model. Journal of College Student Retention: Research, Theory \& Practice. doi:1521025117696822

Turner, P., \& Thompson, E. (2014). College retention initiatives meeting the needs of millennial freshman students. College student journal, 48(1), 94-104.

VanderSchee, B. A. (2009). Embracing enrollment management: A comprehensive approach to college student marketing. Academy of Marketing Studies Journal, $13(1)$.

Vianden, J., \& Barlow, P. J. (2014). Showing the love: Predictors of student loyalty to undergraduate institutions. Journal of Student Affairs Research and Practice, 51(1), 16-29.

Voyer, D., \& Voyer, S. D. (2014). Gender differences in scholastic achievement: A metaanalysis. Psychological Bulletin, 140(4), 1174.

Webber, D. A. (2016). Are college costs worth it? How ability, major, and debt affect the returns to schooling. Economics of Education Review, 53, 296-310.

Weber, M. (1947). The theory of social and economic organization (T. Parsons, Trans.). New York, NY: Free Press.

Weiss, M., Razinskas, S., Backmann, J., \& Hoegl, M. (2018). Authentic leadership and leaders' mental well-being: An experience sampling study. The Leadership Quarterly, 29(2), 309-321. 
Wibrowski, C. R., Matthews, W. K., \& Kitsantas, A. (2017). The role of a skills learning support program on first-generation college students' self-regulation, motivation, and academic achievement: A longitudinal study. Journal of College Student Retention: Research, Theory, Practice, 19(3), 317-332.

WICHE. (2019). Retrieved from https://knocking.wiche.edu/

Williams, J. (2016). A critical exploration of changing definitions of public good in relation to higher education. Studies in Higher Education, 41(4), 619-630.

Witteveen, D., \& Attewell, P. (2017). The college completion puzzle: A hidden markov model approach. Research in Higher Education, 58(4), 449-467.

Young, J. W., \& Johnson, P. M. (2004). The impact of an SES-based model on a college's undergraduate admissions outcomes. Research in Higher Education, 45(7), 777-797. 


\section{APPENDICIES}

APPENDIX A

\section{Informed Consent}

1. IRB letter of approval

2. Gatekeeper Permission Letter for University Employee/Student Participation

3. Gatekeeper Permission for University Employee/Student Participation

4. Letter of Informed Consent -Interview Participant

5. Letter of Informed Consent - Focus Group Participant 


\section{IRB Letter of Approval}

May 14, 2019

Principal Investigator: Justin D. Gragg (MU-Student)

Department: Educational Leadership-EDD

Your IRB Application to project entitled AN EXAMINATION OF ORGANIZATIONAL LEARNING AND DATA DRIVEN DECISION MAKING WITHIN TWO UNIVERSITY ENROLLMENT

MANAGEMENT PROGRAMS was reviewed and approved by the MU Institutional Review Board according to the terms and conditions described below:

$\begin{array}{ll}\text { IRB Project Number } & 2014814 \\ \text { IRB Review Number } & 248179 \\ \text { Initial Application Approval Date } & \text { May 14, 2019 } \\ \text { IRB Expiration Date } & \text { May 14, 2020 } \\ \text { Level of Review } & \text { Exempt } \\ \text { Project Status } & \text { Active - Exempt } \\ \begin{array}{l}\text { Exempt Categories (Revised Common } \\ \text { Rule) }\end{array} & \text { 45 CFR 46.104d(2) } \\ \text { Risk Level } & \text { Minimal Risk }\end{array}$

The principal investigator (PI) is responsible for all aspects and conduct of this study. The PI must comply with the following conditions of the approval:

1. No subjects may be involved in any study procedure prior to the IRB approval date or after the expiration date.

2. All changes must be IRB approved prior to implementation utilizing the Exempt Amendment Form.

3. The Annual Exempt Form must be submitted to the IRB for review and approval at least 30 days prior to the project expiration date to keep the study active or to close it.

4. Maintain all research records for a period of seven years from the project completion date.

If you have any questions or concerns, please contact the MU IRB Office at 573-882-3181 or email to muresearchirb@missouri.edu.

Thank you,

MU Institutional Review Board 


\section{Gatekeeper Permission Letter for University Employee Participation}

\section{Dear $<$ name $>$}

I would like to request your permission to invite applicable employees and student workers from your Office of Undergraduate Admissions to participate in a research study entitled: An Initial Examination of Organizational Learning and Data Driven Decision making within University Enrollment Management. I am examining the ability of an organization to change and learn to use data driven decision-making leading to successful practices in undergraduate admission and enrollment management. Interviews will be coded to determine the level of learning and understanding within key members of the admissions team and how data driven decision making is used in practice. The information gathered should be beneficial to your office for informing potential areas of training and improvement, along with gaining a clear understanding of the understanding and engagement of your employees. This study is part of my dissertation research for a doctoral degree in Educational Leadership and Policy Analysis from the University of Missouri.

For this study, I will interview a senior administrator in Enrollment Management (VP, AVP), the Director of Admissions, a mid-level manager, preferably one who handles data and/or operations, and an admissions representative/recruiter. Additionally, I will conduct a focus group with student workers from your office. Questions will guide the subjects on explaining how their institution used organizational learning to improve their ability to recruit and retain students. Questions will also focus on the data driven decision-making model and how that affects enrollment. I am seeking your permission, as the Associate Vice President for Enrollment Management to contact your admissions office and allow me to work with your admissions office to conduct this research. A copy of the interview protocol and informed consent forms are attached for your review.

Participation in this study in completely voluntary. The participants may withdraw from participation at any time they wish without penalty, including during an interview, if they so choose. Participants' answers will remain confidential, anonymous, and separate from any identifying information. The researcher will not list any names of participants in his dissertation or any future publications of this study.

Please feel free to contact me with any questions or concerns about participation either by phone at (806) 778-0076 or by e-mail at jdgragg1@gmail.com. In addition, you are also welcome to contact the dissertation advisor for this research study, Dr. Barbara Martin, at bmartin@ucmo.edu. If you choose to allow me to contact the admissions office regarding participation in this study, please complete the attached permission form. You should retain a copy of this letter and your written consent for future reference.

Thank you for your time and consideration.

Sincerely, 


\section{J.D. Gragg}

Doctoral Candidate

\section{Gatekeeper Permission for University Employee/Student Participation}

$\mathrm{I}$,

for employees of the

, grant permission

admissions office to be contacted to participate in the study, An Initial Examination of

Organizational Learning and Data Driven Decision making within University Enrollment

Management conducted by J.D. Gragg, doctoral candidate at the University of Missouri.

By signing this permission form, I understand that the following safeguards are in place to protect faculty or staff choosing to participate:

- All participation is voluntary, and may be withdrawn at any point before the culmination of the study.

- All responses will be used for dissertation research and for potential future journal publications.

- All identities will be kept confidential in all phases of the research.

- An interview will occur with each staff member, in person, lasting approximately one hour in length.

Please keep the consent letter and a copy of the signed consent form for your records. If you choose to grant permission for staff in your admissions office to participate in this study, please complete this Administrative Permission for Program Participation Form, and please return it to J.D. Gragg as soon as possible.

I have read the material above, and any questions that I have posed have been answered to my satisfaction. I grant permission for staff in my admissions office to be contacted and invited to participate in this study.

Signed:

Date:

Title/Position:

Institution:

Please return to: J.D. Gragg, 13102 Anthony Drive, St. George, KS 66535 Cell Phone: 806-778-0076 Email: jdgragg1@gmail.com 


\section{Interview Informed Consent Script for University Employee}

You are being asked to participate in a research study, An Initial Examination of Organizational Learning and Data Driven Decision making within University Enrollment Management conducted by J.D. Gragg, doctoral candidate at the University of Missouri.

- Your participation is voluntary, and you can stop participating at any time and do not have to answer any question that you do not feel comfortable answering.

- Your responses will be used for dissertation research and for potential future journal publications.

- Your identity will be kept confidential in all phases of the research.

- The interview will take place either in person, via video teleconferencing, or by phone and should take approximately one hour to complete

- For any future questions, please contact the researcher at:

Cell Phone: 806-778-0076 Email: jdgragg1@gmail.com 


\section{Focus Group Informed Consent Form}

I am agreeing to participate in the study, An Initial Examination of Organizational Learning and Data Driven Decision making within University Enrollment Management conducted by J.D. Gragg, doctoral candidate at the University of Missouri.

As part of this research, the following holds true:

- My participation is voluntary, and may be withdrawn at any point before or during the study

- My responses will be used for dissertation research and for potential future journal publications.

- My identity will be kept confidential in all phases of the research.

- The focus group will take place either in person and should take approximately one hour to complete

- I confirm that I voluntarily agree to participate in this study, and I have been read the material above. All questions that I have posed have been answered to my satisfaction.

- For any future questions, please contact the researcher at:

Cell Phone: 806-778-0076 Email: jdgragg1@gmail.com 


\section{APPENDIX B}

1. Interview Protocol for Admissions Staff

2. Focus Group with students protocol

3. Focus Group with enrollment management staff protocol 


\section{Interview Protocol Admissions Staff}

Admissions Staff Interview Protocol

Date:

Start Time:

Interviewee number:

Place:

Introduction:

Hello. Thank you for taking the time to answer my questions regarding organizational change and data driven decision making within enrollment management. The purpose of today's interview is to gather information from you about your experiences within enrollment management, utilizing data for decision making, and how your organization has changed in your time working here. My name is J.D. Gragg, and I will be conducting the interview. To ensure accuracy, I will be audio taping the interview.

Remember, there are no right or wrong answers. If you want to follow up on a question or give an example, feel free to do so. I want this to be more of a conversation between professionals than an interview. Our session will last a maximum of one hour and we will not be taking a formal break. If you need a break, just let me know. Let's begin by finding out more about each other.

\begin{tabular}{|l|l|}
\hline $\begin{array}{l}\text { 1. } \begin{array}{l}\text { Tell me your name and a little bit } \\
\text { about yourself and your job with X } \\
\text { University. }\end{array} \\
\text { 2. } \begin{array}{l}\text { How long have you been working } \\
\text { for X University? }\end{array}\end{array}$ \\
\hline 3. $\begin{array}{l}\text { In what ways do you use data to } \\
\text { make decisions in your daily work } \\
\text { duties? Are there specific events or } \\
\text { student types that you use data to } \\
\text { recruit more than others? }\end{array}$ & Q 1, Q 4 \\
\hline 4. $\begin{array}{l}\text { How does the organization } \\
\text { (admissions office/enrollment } \\
\text { management as a whole) use data to } \\
\text { inform their policies and practices? }\end{array}$ & \\
\hline 5. $\begin{array}{l}\text { How would you say your } \\
\text { organization has changed during } \\
\text { your time here? Do you use data } \\
\text { more or less? }\end{array}$ & Q 1, Q 2, Q 3 3 \\
\hline
\end{tabular}




\begin{tabular}{|c|c|}
\hline $\begin{array}{l}\text { 6. What practices or procedures are in } \\
\text { place in your office to allow } \\
\text { change/learning to happen? Are } \\
\text { these processes encouraged/created } \\
\text { by your leader? }\end{array}$ & Q 1, Q 2, Q 3 \\
\hline $\begin{array}{l}\text { 7. Please describe your understanding } \\
\text { and use of predictive modeling } \\
\text { within your office. Have you been } \\
\text { involved with the creation of a } \\
\text { predictive model? If so, how? }\end{array}$ & Q 3 \\
\hline $\begin{array}{l}\text { 8. Let us look outside of data for a } \\
\text { minute. What other } \\
\text { resources/factors do you use in your } \\
\text { recruitment process? }\end{array}$ & Q 4 \\
\hline $\begin{array}{l}\text { 9. Please describe your recruitment } \\
\text { efforts and results now as compared } \\
\text { to before you used a more data } \\
\text { driven approach. Are there areas } \\
\text { and/or specific student types you } \\
\text { can recruit better with a data-driven } \\
\text { approach? If so, describe. Is there } \\
\text { an area where you lack data that } \\
\text { you think you could be more } \\
\text { effective with more data? If so, } \\
\text { describe. } \\
\text { 10. Please describe any examples } \\
\text { where you have taken a personal } \\
\text { experience or idea and } \\
\text { implemented a change within the } \\
\text { organization. } \\
\text { 11. Is there anything else you would } \\
\text { like to add that we didn't cover? }\end{array}$ & Q1-4 \\
\hline
\end{tabular}




\section{Focus Group Protocol - Students}

Recently Admitted Students Focus Group Protocol

Date:

Start Time:

Place:

Introduction:

Hello. Thank you for taking the time to discuss your recruitment process to X University. The purpose of today's focus group is to gather information from you about your experiences with the admissions office here at X University, how that compared to how you were recruited by other universities, and what you feel was most effective or impactful to you in making your college decision. My name is J.D. Gragg, and I will be moderating the focus group. To ensure accuracy, I will be audio taping the conversation.

Remember, there are no right or wrong answers. If you want to follow up on a question or give an example, feel free to do so. I want this to be a conversation, please feel free to expand on other participants answers and give examples. Our session will last a maximum of one hour and we will not be taking a formal break. If you need a break, just let me know. Let's begin by finding out more about each other.

\begin{tabular}{|c|c|}
\hline $\begin{array}{l}\text { 1. Tell me your names and a little bit } \\
\text { about yourself. } \\
\text { 2. How long have you been attending } \\
\text { X University? }\end{array}$ & Learn about the participants. \\
\hline $\begin{array}{l}\text { 3. Please describe your experience } \\
\text { being recruited to college in } \\
\text { general. }\end{array}$ & Q 1-4 \\
\hline $\begin{array}{l}\text { 4. How would you say the process of } \\
\text { being recruited to X University } \\
\text { differed from others? Would you } \\
\text { say it was more or less targeted or } \\
\text { specific than other schools? If so, } \\
\text { please describe how. }\end{array}$ & Q 4 \\
\hline $\begin{array}{l}\text { 5. Were any of you recruited more } \\
\text { than once to X University? } \\
\text { Meaning, did you apply as one } \\
\text { student type or for one term, then } \\
\text { not attend, and eventually come } \\
\text { back? If so, how did the }\end{array}$ & Q 2 and Q 4 \\
\hline
\end{tabular}




\begin{tabular}{|l|l|}
\hline $\begin{array}{l}\text { recruitment process change from } \\
\text { the first to the second time? }\end{array}$ & \\
\hline $\begin{array}{l}\text { 6. } \begin{array}{l}\text { How do you feel about the amount } \\
\text { of data higher education gathers on } \\
\text { you in high school and how much } \\
\text { communication they send you? }\end{array} \\
\text { Please describe. }\end{array}$ & \\
\hline 7. $\begin{array}{l}\text { What effect, if any, did X } \\
\text { Universities recruitment efforts } \\
\text { have on your decision to attend } \\
\text { there? }\end{array}$ & Q 1-4 \\
8. Please describe any examples \\
where you have taken a personal \\
experience or idea and \\
implemented a change within the \\
organization.
\end{tabular}




\section{Focus Group Protocol - Enrollment management staff}

Recently Admitted Students Focus Group Protocol

Date:

Start Time:

Place:

Introduction:

Hello. Thank you for taking the time to discuss your role within enrollment management at University X. The purpose of today's focus group is to gather information from you about your experiences within the enrollment management team here at X University. My name is J.D. Gragg, and I will be moderating the focus group. To ensure accuracy, I will be audio taping the conversation.

Remember, there are no right or wrong answers. If you want to follow up on a question or give an example, feel free to do so. I want this to be a conversation, please feel free to expand on other participants answers and give examples. Our session will last a maximum of one hour and we will not be taking a formal break. If you need a break, just let me know. Let's begin by finding out more about each other.

\begin{tabular}{|l|l|}
\hline 6. $\begin{array}{l}\text { Tell me your names and a little } \\
\text { bit about yourself. }\end{array}$ & Learn about the participants. \\
\begin{tabular}{l} 
7. $\begin{array}{l}\text { How long have you been } \\
\text { working X University? }\end{array}$ \\
\hline 8. $\begin{array}{l}\text { Please describe your position } \\
\text { and job duties. }\end{array}$
\end{tabular} & Learn about the participants \\
\hline \begin{tabular}{l} 
9. $\begin{array}{l}\text { Have you worked outside of } \\
\text { enrollment management at this } \\
\text { university or worked at other } \\
\text { colleges or universities in any } \\
\text { capacity? If so, how does your } \\
\text { experience here compare? }\end{array}$ \\
\hline $\begin{array}{l}\text { 10. In what ways do you data in } \\
\text { your day to day work? }\end{array}$
\end{tabular} & Q 1 -3 \\
\hline $\begin{array}{l}\text { 11. Do you feel that the } \\
\text { organization has changed in } \\
\text { regards to the use of data in } \\
\text { your time here? If so, in what } \\
\text { ways? }\end{array}$ & Q 1-4 \\
\hline
\end{tabular}




\begin{tabular}{|l|l|}
\hline $\begin{array}{l}\text { 12. How does leadership use the } \\
\text { ideas or results of data that you } \\
\text { bring to them? Has this } \\
\text { changed? Have the types of } \\
\text { data or ideas being presented } \\
\text { changed? }\end{array}$ & Q \\
\hline $\begin{array}{l}\text { 13. Please describe any examples } \\
\text { where you have taken a } \\
\text { personal experience or idea and } \\
\text { implemented a change within } \\
\text { the organization. }\end{array}$ & Q 2 \\
\hline 14. Is there anything else you \\
would like to add that we didn't \\
cover?
\end{tabular}




\section{Vitae}

Born and raised in Nebraska, J.D. Gragg has worked in higher education since 2006. Upon graduation from the Univeristy of Nebraska at Kearney in 2002 with dual Bachelors degrees in Criminal Justice and Psychology, J.D. went to work for the Lancaster County Department of Corrections in Lincoln, NE. J.D. worked here until 2004 when he went to work as a general manager of a restaurant in Omaha,NE. In 2006, J.D. moved to Lubbock, TX and began his career in higher education. This career change led J.D. to pursue a Masters Degree in Organizational Leadership from Lubbock Christian University in 2013. Continuing his education, J.D. obtained a Doctorate in Educational Leadership and Policy Analysis from the University of Missouri in 2019.

J.D.'s work in higher education began with managing a Kaplan Test Prep Center in Lubbock, where he made connections with the Texas Tech University Advising Center. From Kaplan, he transitioned to become the Pre-Law advisor at TTU. From there, he moved up to become the Director of Recruitment at the Texas Tech University School of Law, overseeing admissions and the national recruitment of students. After a year working for the federal court system in Lubbock, J.D. again returned to higher education as the founding Transfer Recruitment Manager for Undergraduate Admissions at Texas Tech. This job lasted from 2011 to 2013 when J.D. moved with his family to Kansas and he began working as the Articulation Development Coordinator at Johnson County Community College. J.D. held this position for nearly three years before moving on to become the Director of Admissions at the University of Central Missouri. In the summer of 2018, another family moved caused J.D. to take on a position as an Enrollment Consultant with NRCCUA, a job which he holds today. Within these roles, J.D. has 
presented locally to nationally, with countless high school and college presentations, regional conference and professional presentations, as well as national presenations at the National Assocaition of College Admissions Counselors (NACAC) annual conference and the National Institute for the Study of Transfer Students (NISTS) annual conference. J.D. plans to use the research from this dissertation and the experiences gained from this program to help college and universities better serve students to help with the goal of access to college for all who desire it. J.D. currently resides outside of Manhattan, KS with his wife and two sons. 